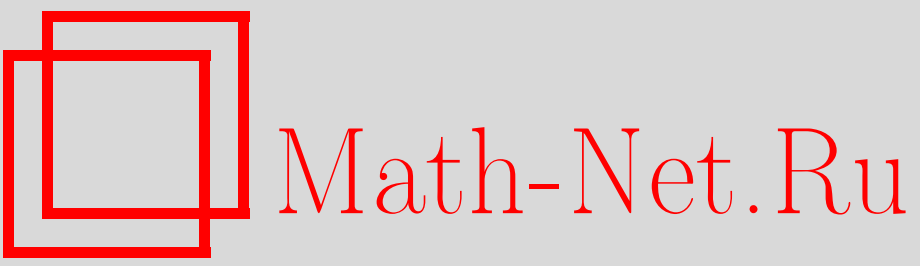

А. А. Карацуба, М. А. Королёв, Аргумент дзета-функции Римана, УМН, 2005, том 60, выпуск 3, 41-96

DOI: https://doi.org/10.4213/rm1429

Использование Общероссийского математического портала Math-Net.Ru подразумевает, что вы прочитали и согласны с пользовательским соглашением

http://www.mathnet.ru/rus/agreement

Параметры загрузки:

IP : 52.6 .47 .48

26 апреля 2023 г., $10: 45: 16$ 
УДК 511

\title{
АРГУМЕНТ ДЗЕТА-ФУНКЦИИ РИМАНА
}

\author{
А. А. КАРАЦУБА, М. А. КОРОЛЁВ
}

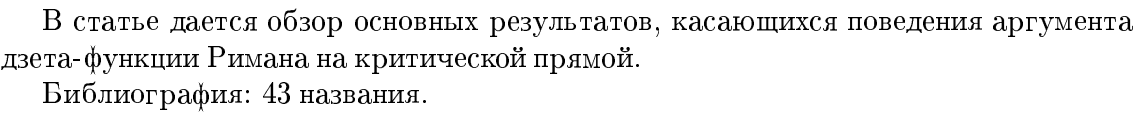

\section{СОДЕРЖАНИЕ}

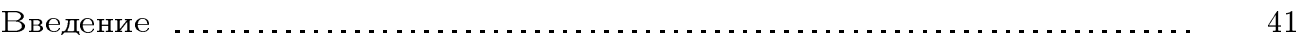

Глава I. Функция $S(t)$ и формула Римана-Мангольдта $\ldots . . . . . . . . . . . . .43$

$\S 1$. Определение функций $S(t), S_{1}(t), N(T)$ и $N(\sigma, T) \ldots \ldots \ldots \ldots \ldots \ldots \ldots \ldots . .43$

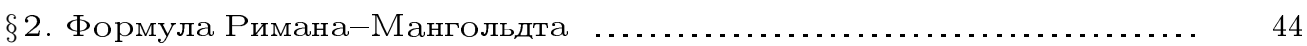

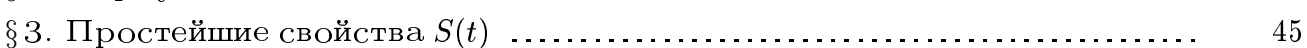

Замечания

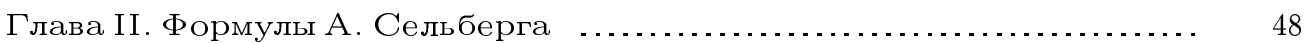

$\S 1$. Приближение функций $S(t)$ и $S_{1}(t)$ отрезками рядов Дирихле $\ldots . . . . .49$

$\S 2$. Несобственный интеграл, содержащий функцию $S(t) \ldots \ldots \ldots \ldots \ldots \ldots .6$

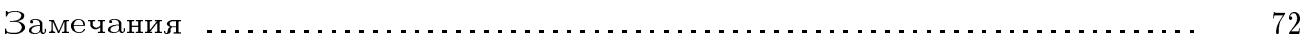

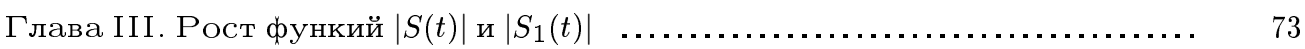

$\S 1$. Верхние оценки $|S(t)|$ и $\left|S_{1}(t)\right|$. Следствия гипотез Римана и Линделё-

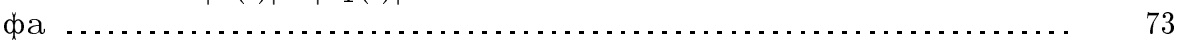

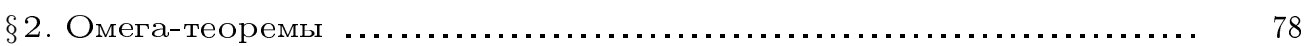

Замечания

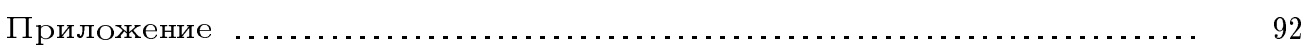

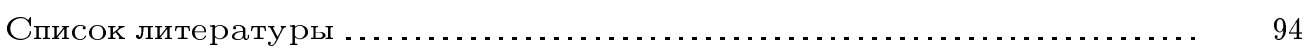

\section{Введение}

В теории дзета-функции Римана $\zeta(s)$ существует три больших направления исследований, в числе которых: 1$)$ нули $\zeta(s)$ в критической полосе $(0 \leqslant \operatorname{Re} s \leqslant 1)$ и на критической прямой $(\operatorname{Re} s=1 / 2), 2)$ порядок роста $|\zeta(s)|$ в критической полосе и на критической прямой и 3$)$ поведение аргумента $\zeta(s)$ на критической прямой.

Первые два направления тесно связаны с рядом центральных проблем теории простых чисел и достаточно хорошо изучены (см. обзоры [1], [2] и библиографии в конще 
каждого из них). Третье направление изучено меньше, хотя и представляет большой интерес как чисто теоретико-функциональное свойство $\zeta(s)$, а также в силу формулы Римана-Мангольдта, служащей полезным инструментом для приближенного вычисления комплексных нулей дзета-функции.

В предлагаемой работе авторы предпринимают попытку восполнить этот пробел и дать систематическое изложение теории функции

$$
S(t)=\frac{1}{\pi} \arg \zeta\left(\frac{1}{2}+i t\right) .
$$

Обзор состоит из трех глав. В первой главе дается определение функции $S(t)$ и приводится доказательство формулы Римана-Мангольдта, которая позволяет исследовать простейшие свойства $S(t)$. Во второй главе излагаются классические формулы А. Сельберга, дающие точные и приближенные выражения для $S(t)$ и связанных с ней функций. Третья глава посвящена изучению порядка роста $S(t)$ и ее первообразной $S_{1}(t)$ при $t \rightarrow+\infty$. В ней наряду с безусловньми результатами помешены утверждения, доказательства которых используют недоказанные в настоящее время гипотезы Линделёфа и Римана.

Каждая глава обзора имеет свою нумерацию определений, лемм и теорем, а также снабжена небольшим комментарием, содержащим ссылки на оригинальные работы. В приведенном списке литературы можно найти дополнительные сведения, касаюшиеся рассматриваемых в статье вопросов (см. [3]-[15]).

В работе используются следуюшие обозначения.

$C, c, c_{1}, c_{2}, \ldots$ - абсолютные положительные постоянные, в разных соотношениях, вообше говоря, разные;

$\theta, \theta_{1}, \theta_{2}, \ldots$ - комплексные числа, по модулю не превосходяшие единицы и в разных соотношениях разные;

символами $p, p_{1}, p_{2}, \ldots, q, q_{1}, q_{2}, \ldots$ обозначаются простые числа;

постоянные в знаках $O$ и $\ll-$ абсолютные, если не оговорено противное;

моментом степени $\alpha, \alpha \geqslant 0$, функции $f(u)$, определенной на промежутке $a \leqslant u \leqslant b$, будем назьвать интеграл

$$
\int_{a}^{b}|f(u)|^{\alpha} d \alpha
$$

если $\alpha=2 k$, где $k=1,2, \ldots$, то соответствуюшие интегралы будем называть четными моментами $f$, а если $\alpha$ не является цельм числом - дробными моментами $f$;

интегралом в смысле главного значения от функции $g(u)$, имеющей особенности в точках $a_{1}, a_{2}, \ldots, a_{n}$ промежутка $a<u<b$, конечного или бесконечного, будем называть предел при $\varepsilon \rightarrow+0$ величины

$$
\left(\int_{a}^{a_{1}-\varepsilon}+\int_{a_{1}+\varepsilon}^{a_{2}-\varepsilon}+\cdots+\int_{a_{n-1}+\varepsilon}^{a_{n}-\varepsilon}+\int_{a_{n}+\varepsilon}^{b}\right) g(u) d u
$$

и обозначать его

$$
\text { v.p. } \int_{a}^{b} g(u) d u
$$

$(a, b)$ - наибольший обший делитель натуральных чисел $a$ и $b$; 
$\mu(n)$ - функция Мёбиуса,

$\mu(n)= \begin{cases}1, & \text { если } n=1 ; \\ 0, & \text { если } n \text { делится на квадрат простого числа; } \\ (-1)^{r}, & \text { если } n=p_{1} \cdots p_{r}, \text { где } p_{1}, \ldots, p_{r}-\text { различные простые числа; }\end{cases}$

$\Lambda(n)$ - функция Мангольдта, равная $\ln p$, если $n=p^{r}, p$ - простое, $r \geqslant 1$ - целое, и равная нулю в остальных случаях;

$[x]$ - целая часть $x$, т.е. наименьшее целое, не превосходящее $x ;\{x\}$ - дробная часть $x$, $\{x\}=x-[x]$

$\mathbf{E}$ - постоянная Эйлера,

$$
\mathbf{E}=\lim _{N \rightarrow+\infty}\left(\sum_{n=1}^{N} \frac{1}{n}-\ln N\right)=0.5772146 \ldots
$$

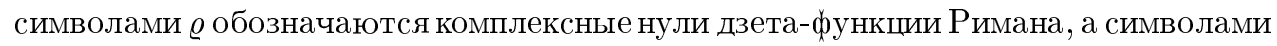
$\beta$ и $\gamma-$ их вешественные и мнимые части (если не оговорено противное).

Запись “лемма $\alpha \Pi ”$ означает ссылку на лемму $\alpha$ приложения.

При необходимости в начале глав вводятся дополнительные обозначения.

\section{Глава I.}

\section{Функция $S(t)$ и формула Римана-Мангольдта}

\section{$\S$ 1. Определение функций $S(t), S_{1}(t), N(T)$ и $N(\sigma, T)$}

Дадим определение функщии $S(t)$, ее первообразной $S_{1}(t)$ и классических в рассматриваемых вопросах функций $N(T), N(\sigma, T)$.

ОПРЕДЕЛЕНИЕ 1 . Для вещественного $t$, отличного от ординаты нуля $\zeta(s)$, положим

$$
S(t)=\frac{1}{\pi} \arg \zeta\left(\frac{1}{2}+i t\right)
$$

где $\arg \zeta(1 / 2+i t)$ получается непрерьвным продолжением $\arg \zeta(s)$ вдоль ломаной линии, начинаюшейся в точке $s=2(\arg \zeta(2)=0)$, идушей к точке $s=2+i t$ и затем к точке $s=1 / 2+i t$. Если же $t-$ мнимая часть нуля $\zeta(s)$, то

$$
S(t)=\lim _{\delta \rightarrow+0} \frac{1}{2}(S(t+\delta)+S(t-\delta))
$$

ОПРЕДЕЛЕНИЕ 2. При положительном числе $t$ функция $S_{1}(t)$ определяется равенством

$$
S_{1}(t)=\int_{0}^{t} S(u) d u \text {. }
$$


ОПРЕДЕЛЕНИЕ 3. Для положительного $T$, отличного от мнимой части нуля $\zeta(s)$, символом $N(T)$ будем обозначать число нулей дзета-функции в прямоугольнике $0 \leqslant \operatorname{Re} s \leqslant 1,0<\operatorname{Im} s \leqslant T$. Если $T$ совпадает с мнимой частью нуля $\zeta(s)$, то положим

$$
N(T)=\lim _{\delta \rightarrow+0} \frac{1}{2}(N(T+\delta)+N(T-\delta))=\frac{1}{2}(N(T+0)+N(T-0)) .
$$

ОПРЕДЕЛЕНИЕ 4. При положительном числе $T$ и $\sigma>0$ символом $N(\sigma, T)$ будем обозначать количество нулей $\zeta(s)$ в прямоугольнике $\sigma<\operatorname{Re} s \leqslant 1,0<\operatorname{Im} s \leqslant T$.

В следуюшем параграфе устанавливается связь между функциями $S(T)$ и $N(T)$.

\section{§ 2. Формула Римана-Мангольдта}

У тверждение теоремы этого параграфа носит название формулы Римана-Мангольдта.

ТеОРема 1. Для любого $T \geqslant 1$ справедливо равенство

$$
N(T)=\frac{T}{2 \pi} \ln \frac{T}{2 \pi}-\frac{T}{2 \pi}+\frac{7}{8}+S(T)+\frac{1}{\pi} \delta(T),
$$

əде

$$
\delta(T)=\frac{T}{4} \ln \left(1+\frac{1}{4 T^{2}}\right)+\frac{1}{4} \operatorname{arctg} \frac{1}{2 T}-\frac{T}{2} \int_{0}^{+\infty} \frac{\varrho(u) d u}{(u+1 / 4)^{2}+(T / 2)^{2}}
$$

$a \varrho(u)=1 / 2-\{u\}$.

ДокаЗАТЕЛЬСТвО. Пусть $T$ не равно мнимой части нуля $\zeta(s)$. Поскольку комплексные нули дзета-функции совпадают с нулями целой функции

$$
\xi(s)=s(s-1) \pi^{-s / 2} \Gamma(s / 2) \zeta(s)
$$

(см. леммы $1 П$ и $2 П$ ), то в силу принципа аргумента (лемма $3 П)$ величина $2 \pi N(T)$ равна прирашению аргумента $\xi(s)$ вдоль границы прямоугольника с вершинами в точках $2,2+i T,-1+i T,-1$.

Так как $\xi(s)$ вещественна при вещественных $s$, то прирашение аргумента $\xi(s)$ вдоль нижней стороны прямоугольника равно нулю.

Кроме того, из равенства $\xi(s)=\bar{\xi}(1-\bar{s})$ (см. лемму 1П) следует, что приращение $\arg \xi(s)$ при изменении $s$ от $s=2$ к $s=2+i T$ и далее к $s=1 / 2+i T$ равно приращению, которое получает $\arg \xi(s)$ при движении $s$ по симметричному контуру от $s=1 / 2+i T$ к $s=-1$ через точку $s=-1+i T$. Следовательно,

$$
N(T)=\frac{1}{\pi} \Delta_{L} \arg \xi(s)
$$


где $\Delta_{L} \arg \xi(s)$ означает прирашение $\arg \xi(s)$ вдоль ломаной $L$ с концами в точках $s=2, s=2+i T$ и $s=1 / 2+i T$. Пользуясь формулой Стирлинга для логарифма гамма-функции (лемма $4 \Pi$ ) наряду с определением $S(T)$, находим:

$$
\begin{aligned}
N(T)= & \frac{1}{\pi}\left(\Delta_{L} \arg s(s-1)+\Delta_{L} \arg \pi^{-s / 2}+\Delta_{L} \arg \Gamma(s / 2)+\pi S(T)\right) \\
= & \frac{1}{\pi}\left(\pi-\frac{T}{2} \ln \pi+\operatorname{Im} \ln \Gamma\left(\frac{1}{4}+\frac{i T}{2}\right)+\pi S(T)\right) \\
= & \frac{1}{\pi}\left(\pi-\frac{T}{2} \ln \pi+\frac{T}{4} \ln \left(\frac{T^{2}}{4}+\frac{1}{16}\right)-\frac{1}{4}\left(\frac{\pi}{2}-\operatorname{arctg} \frac{1}{2 T}\right)-\frac{T}{2}\right. \\
& \left.-\frac{T}{2} \int_{0}^{+\infty} \frac{\varrho(u) d u}{(u+1 / 4)^{2}+(T / 2)^{2}}+\pi S(T)\right)=L(T)+S(T)+\frac{1}{\pi} \delta(T),
\end{aligned}
$$

где обозначено:

$$
\begin{gathered}
L(T)=\frac{T}{2 \pi} \ln \frac{T}{2 \pi}-\frac{T}{2 \pi}+\frac{7}{8} \\
\delta(T)=\frac{T}{4} \ln \left(1+\frac{1}{4 T^{2}}\right)+\frac{1}{4} \operatorname{arctg} \frac{1}{2 T}-\frac{T}{2} \int_{0}^{+\infty} \frac{\varrho(u) d u}{(u+1 / 4)^{2}+(T / 2)^{2}},
\end{gathered}
$$

$\varrho(u)=1 / 2-\{u\}$.

Пусть теперь $T$ совпадает с ординатой одного из нулей $\zeta(s)$. По доказанному выше, для достаточно малых $h, h>0$,

$$
N(T \pm h)=L(T \pm h)+S(T \pm h)+\pi^{-1} \delta(T \pm h)
$$

Сложим эти равенства. Устремляя $h$ к нулю и пользуясь непрерьвностью функций $L(T)$ и $\delta(T)$, будем иметь:

$$
\begin{aligned}
N(T) & =\lim _{h \rightarrow+0} \frac{1}{2}(N(T+h)+N(T-h)) \\
& =L(T)+\lim _{h \rightarrow+0} \frac{1}{2}(S(T+h)+S(T-h))+\pi^{-1} \delta(T)=L(T)+S(T)+\pi^{-1} \delta(T) .
\end{aligned}
$$

Теорема доказана.

\section{$\S$ 3. Простейшие свойства $S(t)$}

Следствием формулы Римана-Мангольдта является приводимая ниже теорема 2.

Теорема 2. Справедливы следующие утверждения.

1. $S(t)$ - кусочно-гладкая функиия с разрывами в точках, совпадающих с ординатами комплексных нулей $\zeta(s)$.

2. При переходе через точку разрыва $S(t)$ совершает скачок, равный сумме кратностей нулей $\zeta(s)$, имеющих эту точку своей ординатой.

3. На всяком промехутке непрерывности $\left(\gamma, \gamma^{\prime}\right)$, где $\gamma, \gamma^{\prime}$ - соседние ординаты нулей $\zeta(s)$, функция $S(t)$ является монотонно убывающей с производнылми

$$
S^{\prime}(t)=-\frac{1}{2 \pi} \ln \frac{t}{2 \pi}+O\left(t^{-2}\right) \quad u \quad S^{\prime \prime}(t)=-\frac{1}{2 \pi t}+O\left(t^{-3}\right) .
$$


ДокаЗАтельство. Докажем лишш последнее утверждение. Пусть $\gamma<t<\gamma^{\prime}$. Тогда на всем интервале $N(t) \equiv N(\gamma+0)$ - постоянная величина. Дифференцируя обе части формулы

$$
S(t)=-\frac{t}{2 \pi} \ln \frac{t}{2 \pi}+\frac{t}{2 \pi}-\frac{7}{8}-\delta(t)+N(\gamma+0),
$$

находим:

$$
S^{\prime}(t)=-\frac{1}{2 \pi} \ln \frac{t}{2 \pi}-\delta^{\prime}(t), \quad S^{\prime \prime}(t)=-\frac{1}{2 \pi t}-\delta^{\prime \prime}(t) .
$$

Перепишем $\delta(t)$ в виде суммы

$$
\frac{t}{4} \ln \left(1+\frac{1}{4 t^{2}}\right)+\frac{1}{4} \operatorname{arctg} \frac{1}{2 t}-\frac{t}{2} j
$$

где

$$
j=j(t)=\int_{0}^{+\infty} \frac{\varrho(u) d u}{(u+1 / 4)^{2}+(t / 2)^{2}} .
$$

Два первых слагаемых в формуле для $\delta(t)$ разлагаются в абсолютно сходящиеся ряды по нечетным степеням $t^{-1}$, так что их вклад в $\delta^{(k)}(t)$ равен $O\left(t^{-k-2}\right), k=0,1,2$. Осталось показать, что для тех же $k j^{(k)}(t)=O\left(t^{-k-2}\right)$. Интегрируя по частям, представим $j$ в виде

$$
\int_{0}^{+\infty} \frac{d \sigma(u)}{(u+1 / 4)^{2}+(t / 2)^{2}}=2 \int_{0}^{+\infty} \frac{\sigma(u)(u+1 / 4) d u}{\left((u+1 / 4)^{2}+(t / 2)^{2}\right)^{2}}, \quad \sigma(u)=\int_{0}^{u} \varrho(z) d z .
$$

Пользуясь неравенством $0 \leqslant \sigma(u) \leqslant 1 / 8$, приходим к оценкам:

$$
\begin{aligned}
|j(t)| & \leqslant \frac{1}{4} \int_{0}^{+\infty} \frac{(u+1 / 4) d u}{\left((u+1 / 4)^{2}+(t / 2)^{2}\right)^{2}} \ll \int_{0}^{t} u t^{-4} d u+\int_{t}^{+\infty} u^{-3} d u \ll t^{-2}, \\
\left|j^{\prime}(t)\right| & =8 \int_{0}^{+\infty} \frac{\sigma(u)(u+1 / 4)^{2} d u}{\left((u+1 / 4)^{2}+(t / 2)^{2}\right)^{3}} \ll \int_{0}^{t} u^{2} t^{-6} d u+\int_{t}^{+\infty} u^{-4} d u \ll t^{-3}, \\
\left|j^{\prime \prime}(t)\right| & =64 \int_{0}^{+\infty} \frac{\sigma(u)(u+1 / 4)^{3} d u}{\left((u+1 / 4)^{2}+(t / 2)^{2}\right)^{4}} \ll \int_{0}^{t} u^{3} t^{-8} d u+\int_{t}^{+\infty} u^{-5} d u \ll t^{-4} .
\end{aligned}
$$

Таким образом,

$$
\begin{aligned}
\delta^{\prime}(t) & =-\frac{1}{2}\left(j(t)+t j^{\prime}(t)\right)+O\left(t^{-2}\right)=O\left(t^{-2}\right), \\
\delta^{\prime \prime}(t) & =-\frac{1}{2}\left(2 j^{\prime}(t)+t j^{\prime \prime}(t)\right)+O\left(t^{-3}\right)=O\left(t^{-3}\right) .
\end{aligned}
$$

Теорема доказана.

\section{Соотношения}

$$
S(t)=O(\ln t), \quad S_{1}(t)=O(\ln t), \quad S(t)=\Omega_{ \pm}\left(\left(\frac{\ln t}{\ln \ln t}\right)^{1 / 3}\right), \quad t \rightarrow+\infty,
$$


доказательства которых будут даны в следующих главах, указьвают на то, что $S(t)$ является сильно осциллирующей функцией. Естественно возник вопрос об изучении количества точек, в которых $S(t)$ меняет знак и которые попадают в заданный промежуток вещественной оси.

Пусть $M(T)$ - число таких точек $t$ с условием $0<t \leqslant T$. Вычисления значений $M(T)$ при малых $T$ дают:

$$
\begin{aligned}
& M(50)=21, \quad M(100)=60, \quad M(150)=106, \\
& M(200)=160, \quad M(250)=217, \quad M(295)=270 .
\end{aligned}
$$

TeOpema 3. $M(T) \rightarrow+\infty$ npu $T \rightarrow+\infty$.

ДоКАЗАТЕльство. Пусть $\gamma_{n}, n=1,2, \ldots,-$ положительные ординаты нулей $\zeta(s)$, занумерованные в порядке возрастания, и пусть $\gamma_{n}<t<\gamma_{n+1}$. Приблизим $S(t)$ линейной функцией

$$
l(t)=\frac{S\left(\gamma_{n+1}-0\right)-S\left(\gamma_{n}+0\right)}{\gamma_{n+1}-\gamma_{n}}\left(t-\gamma_{n}\right)+S\left(\gamma_{n}+0\right),
$$

принимающей на концах интервала значения $S\left(\gamma_{n}+0\right)$ и $S\left(\gamma_{n+1}-0\right)$. Точность такого приближения оценивается с помошњю формулы Тейлора и утверждения п. 3 предыдущей теоремы:

$$
|S(t)-l(t)| \leqslant\left(\gamma_{n+1}-\gamma_{n}\right)^{2} \sup _{\gamma_{n}<t<\gamma_{n+1}}\left|S^{\prime \prime}(t)\right|=O\left(\frac{\left(\gamma_{n+1}-\gamma_{n}\right)^{2}}{\gamma_{n}}\right) .
$$

Из приведенных соотношений следует равенство

$$
\begin{aligned}
\int_{\gamma_{n}}^{\gamma_{n+1}} S(t) d t & =\int_{\gamma_{n}}^{\gamma_{n+1}} l(t) d t+O\left(\frac{\left(\gamma_{n+1}-\gamma_{n}\right)^{3}}{\gamma_{n}}\right) \\
& =\frac{1}{2}\left(\gamma_{n+1}-\gamma_{n}\right)\left(S\left(\gamma_{n+1}-0\right)+S\left(\gamma_{n}+0\right)\right)+O\left(\frac{\left(\gamma_{n+1}-\gamma_{n}\right)^{3}}{\gamma_{n}}\right) .
\end{aligned}
$$

Если теперь предположить, что при $t \geqslant \gamma_{n_{0}-1}$ функция $S(t)$ принимает только положительные значения, то мы придем к неравенствам $S\left(\gamma_{n}-0\right)>0, S\left(\gamma_{n+1}-0\right)>0$, которые справедливы при всех $n \geqslant n_{0}$. Так как в точке разрыва $\gamma_{n}$ функция $S(t)$ совершает скачок на величину, не меньшую 1 , то

$$
S\left(\gamma_{n}+0\right) \geqslant S\left(\gamma_{n}-0\right)+1>1
$$

Поэтому при всех $n \geqslant n_{0}$

$$
\int_{\gamma_{n}}^{\gamma_{n+1}} S(t) d t \geqslant \frac{1}{2}\left(\gamma_{n+1}-\gamma_{n}\right)(1+o(1))+O\left(\frac{\left(\gamma_{n+1}-\gamma_{n}\right)^{3}}{\gamma_{n}}\right)>\frac{1}{3}\left(\gamma_{n+1}-\gamma_{n}\right) .
$$

Беря $N>n_{0}$ и складьвая полученные неравенства с $n=n_{0}, n_{0}+1, \ldots, N-1$, мы придем к соотношению

$$
\int_{\gamma_{n_{0}}}^{\gamma_{N}} S(t) d t \geqslant \frac{1}{3}\left(\gamma_{N}-\gamma_{n_{0}}\right)
$$


Предположение об отрицательности $S(t)$ при больших $t$ приводит к неравенству

$$
\int_{\gamma_{n_{0}}}^{\gamma_{N}} S(t) d t \leqslant-\frac{1}{3}\left(\gamma_{N}-\gamma_{n_{0}}\right)
$$

При больших $N$ это и предыдущее неравенства противоречат оценке

$$
\int_{0}^{t} S(u) d u=O(\ln t)
$$

(см. теорему 2 гл. III). Следовательно, $S(t)$ меняет знак бесконечно много раз. Теорема доказана.

\section{Замечания}

В некоторых работах функщии $S(T)$ и $N(T)$ определяют в точках разрьва как пределы величин $S(T+\delta)$ и $N(T+\delta)$ при $\delta \rightarrow+0$.

Асимптотическая формула для $N(T)$ была впервые доказана Б. Риманом [16]. Различные способы оценки остаточного члена в этой формуле (т.е. фактически оценка $|S(T)|)$ были предложены фон Мангольдтом в 1905 г. (см. [17]) и Р. Бэклундом [18] в 1914 г. (см. также [2; с. 89-90]).

Теорема 3 доказана в 1913 г. Г. Бором и Э. Ландау [17] (см. также [19], [20; с. 222]).

Задача определения правильного порядка роста $M(T)$ при растущем $T$ до концане решена. Современное состояние этого вопроса будет изложено в следующей статье авторов (см. также [21]-[26]).

\section{Глава II. Формулы А. Сельберга}

Одним из основных инструментов при исследовании аргумента дзета-функщии Римана на критической прямой являются “явные” формулы Сельберга, которыеприближают $S(t)$ и $S_{1}(t)$ отрезками рядов Дирихле специального вида.

Эти явные формулы позволяют находить асимптотики моментов $S(t)$, т.е. асимптотики интегралов

$$
\int_{T}^{T+H}|S(t)|^{a} d t, \quad a>0,
$$

которые возникают в задачах о числе перемен знака $S(t)$ и о распределении значений величины $|S(t)|$ на отрезках вешественной прямой. Вьводу формул Сельберга посвящен первый параграф главы.

Во втором параграфе изучаются несобственные интегралы вида

$$
\int_{-\infty}^{+\infty} f(u) S(t+u) d u
$$

Точные выражения для них лежат в основе доказательств омега-теорем для функшии $S(t)$. 


\section{$\S$ 1. Приближение функций $S(t)$ и $S_{1}(t)$ отрезками рядов Дирихле}

Лемма 1. Пусть $m-$ иелое, $m \geqslant 3, t-$ вещественное число, $s=-(m+1 / 2)+i t$. Тогда

$$
\left|\frac{\Gamma^{\prime}(s)}{\Gamma(s)}\right|<3(\ln |s|+3) \text {. }
$$

ДокАЗАТЕЛЬСТво. Так как для любого натурального числа $n$ вьполняются неравенства

$$
|n+s| \geqslant 1 / 2, \quad|n+s| \geqslant \max (n-|s|,|s|-n),
$$

то, пользуясь формулой для логарифмической производной гамма-функции, будем иметь:

$$
\begin{aligned}
\left|\frac{\Gamma^{\prime}(s)}{\Gamma(s)}\right| & =\left|\sum_{n=1}^{+\infty}\left(\frac{1}{n}-\frac{1}{s+n}\right)-\mathbf{E}-\frac{1}{s}\right| \\
& \leqslant \sum_{n \leqslant|s|-1}\left(\frac{1}{n}+\frac{1}{|s|-n}\right)+\left(\frac{2}{|s|-1}+\frac{2}{1 / 2}\right)+\sum_{n>|s|+1} \frac{|s|}{n(n-|s|)}+\mathbf{E}+\frac{1}{3} \\
& <2(\ln |s|+1)+5+\int_{|s|+1}^{+\infty}\left(\frac{1}{u-|s|}-\frac{1}{u}\right) d u+\mathbf{E}+\frac{1}{2}<3(\ln |s|+3) .
\end{aligned}
$$

Лемма доказана.

ЛЕмма 2. Для вещественных $\sigma u t,|\sigma| \leqslant 2,|t| \geqslant 10, s=\sigma+i t$, справедливо следующее равенство:

$$
\frac{\zeta^{\prime}(s)}{\zeta(s)}=\sum_{\varrho} \frac{1}{s-\varrho}+\theta\left(\frac{1}{2} \ln |t|+3\right)
$$

$2 \partial e$

$$
\sum_{\varrho}(s-\varrho)^{-1}=\sum_{\operatorname{Im} \varrho>0}\left((s-\varrho)^{-1}+(s-\bar{\varrho})^{-1}\right)
$$

ДокАЗАТЕльство. Представим функцию Римана $\xi(s)$ в виде произведения Вейерштрасса по нулям дзета-функции (лемма 2П):

$$
\xi(s)=s(s-1) \pi^{-s / 2} \Gamma\left(\frac{s}{2}\right) \zeta(s)=e^{B s} \prod_{\varrho}\left(1-\frac{s}{\varrho}\right) e^{s / \varrho} .
$$

Логарифмируя, а затем дифференцируя это равенство, будем иметь:

$$
\frac{\xi^{\prime}(s)}{\xi(s)}=\left(\frac{\zeta^{\prime}(s)}{\zeta(s)}+\frac{1}{s-1}\right)+\left(\frac{1}{2} \frac{\Gamma^{\prime}(s / 2)}{\Gamma(s / 2)}+\frac{1}{s}\right)-\frac{1}{2} \ln \pi=B+\sum_{\varrho} \frac{1}{\varrho}+\sum_{\varrho} \frac{1}{s-\varrho}
$$

где

$$
\sum_{\varrho} \varrho^{-1}=\sum_{\operatorname{Im} \varrho>0}\left(\varrho^{-1}+\bar{\varrho}^{-1}\right), \quad \sum_{\varrho}(s-\varrho)^{-1}=\sum_{\operatorname{Im} \varrho>0}\left((s-\varrho)^{-1}+(s-\bar{\varrho})^{-1}\right) .
$$


Возьмем в полученном соотношении последовательно $s=0, s=1$ :

$$
\frac{\xi^{\prime}(0)}{\xi(0)}=B, \quad \frac{\xi^{\prime}(1)}{\xi(1)}=B+\sum_{\varrho} \frac{1}{\varrho}+\sum_{\varrho} \frac{1}{1-\varrho}=B+2 \sum_{\varrho} \frac{1}{\varrho} .
$$

Из функционального уравнения $\xi(s)=\xi(1-s)$ (лемма 1П) следует, что

$$
\frac{\xi^{\prime}(0)}{\xi(0)}+\frac{\xi^{\prime}(1)}{\xi(1)}=0, \quad \text { т.е. } 2 B+2 \sum_{\varrho} \frac{1}{\varrho}=0
$$

Таким образом,

$$
\begin{aligned}
\frac{\zeta^{\prime}(s)}{\zeta(s)}-\sum_{\varrho} \frac{1}{s-\varrho} & =-\left(\frac{1}{2} \frac{\Gamma^{\prime}(s / 2)}{\Gamma(s / 2)}+\frac{1}{s}\right)+\frac{1}{s-1}+\frac{1}{2} \ln \pi \\
& =\sum_{n=1}^{+\infty}\left(\frac{1}{s+2 n}-\frac{1}{2 n}\right)+\frac{1}{2}(\mathbf{E}+\ln \pi)+\frac{1}{1-s}
\end{aligned}
$$

Для завершения доказательства осталось оценить правую часть последнего равенства. По абсолютной величине она не превосходит

$$
\begin{aligned}
& \sum_{n \leqslant|t|}\left(\frac{1}{|t|}+\frac{1}{2 n}\right)+\sum_{n>|t|} \frac{|s|}{(2 n)^{2}}+\frac{1}{2}(\mathbf{E}+\ln \pi)+\frac{1}{|t|} \\
& \quad \leqslant 1+\frac{1}{2}(\ln |t|+1)+\frac{1}{4} \frac{|t|+2}{|t|-1}+\frac{1}{2}(\mathbf{E}+\ln \pi)+\frac{1}{|t|}<\frac{1}{2} \ln |t|+3 .
\end{aligned}
$$

Лемма доказана.

Лемма 3. При $|t| \rightarrow+\infty$ справедливо следующее соотношение: $S(t)=O(\ln |t|)$.

ДоКАЗАТЕльство. Поскольку $S(-t)=-S(t)$, то достаточно рассмотреть случай $t \geqslant t_{0}>20$. Пусть сначала $t$ отлично от мнимой части нуля дзета-функции, $s=\sigma+i t$, $1 / 2 \leqslant \sigma \leqslant 2$. Согласно предыдушей лемме,

$$
\begin{aligned}
\frac{\zeta^{\prime}(s)}{\zeta(s)}-\frac{\zeta^{\prime}(2+i t)}{\zeta(2+i t)}= & \sum_{\varrho} \frac{1}{s-\varrho}-\sum_{\varrho} \frac{1}{2+i t-\varrho}+\theta(\ln t+6) \\
= & \sum_{|t-\gamma| \leqslant 1} \frac{1}{s-\varrho}-\sum_{|t-\gamma| \leqslant 1} \frac{1}{2+i t-\varrho} \\
& +\sum_{|t-\gamma|>1} \frac{2-\sigma}{(\sigma-\beta+i(t-\gamma))(2-\beta+i(t-\gamma))}+O(\ln t) .
\end{aligned}
$$

Из простейших теорем о нулях $\zeta(s)$ (см. [2; с. 88]) следует, что число слагаемых в первых двух суммах есть $O(\ln t)$. Так как $|2+i t-\varrho| \geqslant 2-\beta \geqslant 1$, то вторая сумма равна $O(\ln t)$. Последняя, третья сумма по модулю не превосходит величины

$$
\sum_{|t-\gamma|>1} \frac{1}{(t-\gamma)^{2}}=\sum_{n=1}^{+\infty} \sum_{n<|t-\gamma| \leqslant n+1} \frac{1}{(t-\gamma)^{2}}=\sum_{n=1}^{+\infty} O\left(\frac{\ln (t+n)}{n^{2}}\right)=O(\ln t) .
$$


Таким образом,

$$
\frac{\zeta^{\prime}(s)}{\zeta(s)}=\sum_{|t-\gamma| \leqslant 1} \frac{1}{s-\varrho}+\frac{\zeta^{\prime}(2+i t)}{\zeta(2+i t)}+O(\ln t)=\sum_{|t-\gamma| \leqslant 1} \frac{1}{s-\varrho}+O(\ln t)
$$

Интегрируя это равенство по отрезку, соединяющему точки $2+i t$ и $1 / 2+i t$, будем иметь:

$$
\ln \zeta(1 / 2+i t)-\ln \zeta(2+i t)=\sum_{|t-\gamma| \leqslant 1}(\ln (1 / 2+i t-\varrho)-\ln (2+i t-\varrho))+O(\ln t)
$$

Отделив в получившемся соотношении мнимую часть, мы заключаем, что прирашение аргумента $\zeta(s)$ вдоль отрезка интегрирования равно $O(\ln t)$. Поскольку при перемешении $s$ по прямой от точки $s=2$ к точке $s=2+i t \arg \zeta(s)$ получает прирашение, равное

$$
\operatorname{Im}(\ln \zeta(2+i t)-\ln \zeta(2))=-\sum_{n=2}^{+\infty} \frac{\Lambda(n)}{\ln n} \frac{\sin (t \ln n)}{n^{2}}=O(1)
$$

а величина $\pi S(t)$ есть сумма этих двух приращений, то $S(t)=O(\ln t)$.

Если $t$ равно мнимой части нуля $\zeta(s)$, то, по доказанному выше, при достаточно малых положительных $h$ справедливы неравенства:

$$
|S(t+h)| \leqslant C \ln (t+h), \quad|S(t-h)| \leqslant C \ln (t-h),
$$

где $C$ - абсолютная постоянная, $C>0$. Устремляя $h$ к нулю, находим:

$$
|S(t)| \leqslant \frac{1}{2}(|S(t+0)|+|S(t-0)|) \leqslant C \ln t .
$$

Лемма доказана.

СлЕДСТВИЕ. Справедливо следующее соотношение:

$$
N(T)=\frac{T}{2 \pi} \ln \frac{T}{2 \pi}-\frac{T}{2 \pi}+O(\ln T)
$$

ЗАмечАнИЕ. Значение постоянной $C$ в неравенстве $|S(t)| \leqslant C \ln t$ будет уточнено в третьей главе.

Лемма 4. Для любого вещественного $t, t \neq 0$, справедлива следующая формула:

$$
\pi S(t)=-\int_{1 / 2}^{+\infty} \operatorname{Im} \frac{\zeta^{\prime}(\sigma+i t)}{\zeta(\sigma+i t)} d \sigma
$$


ДоКАЗАТЕльСТВо. Обозначим несобственньй интеграл из условия леммы символом $F(t)$.

Пусть $t$ отлично от ординаты нуля $\zeta(s)$. Тогда величина $\pi S(t)$ равна сумме приращений мнимой части $\ln \zeta(s)$ вдоль отрезков, соединяюших точки $2,2+i t$ и $1 / 2+i t$, т.е. сумме

$$
\operatorname{Im}(\ln \zeta(2+i t)-\ln \zeta(2))+\operatorname{Im} \int_{2+i t}^{1 / 2+i t} d \ln \zeta(s)
$$

Разлагая на прямой $\operatorname{Re} s=2 \ln \zeta(s)$ в ряд Дирихле, получим:

$$
\begin{aligned}
\pi S(t) & =-\int_{1 / 2}^{2} \operatorname{Im} \frac{\zeta^{\prime}(\sigma+i t)}{\zeta(\sigma+i t)} d \sigma+\operatorname{Im} \sum_{n=2}^{+\infty} \frac{\Lambda(n)}{\ln n} \frac{1}{n^{2+i t}} \\
& =-\int_{1 / 2}^{2} \operatorname{Im} \frac{\zeta^{\prime}(\sigma+i t)}{\zeta(\sigma+i t)} d \sigma+\operatorname{Im} \sum_{n=2}^{+\infty} \int_{2}^{+\infty} \frac{\Lambda(n)}{n^{\sigma+i t}} d \sigma \\
& =-\int_{1 / 2}^{2} \operatorname{Im} \frac{\zeta^{\prime}(\sigma+i t)}{\zeta(\sigma+i t)} d \sigma-\int_{2}^{+\infty} \operatorname{Im} \frac{\zeta^{\prime}(\sigma+i t)}{\zeta(\sigma+i t)} d \sigma=F(t)
\end{aligned}
$$

Пусть теперь $t$ совпадает с ординатой одного из нулей $\zeta(s)$. Покажем, что

$$
F(t)=\frac{1}{2}(F(t+0)+F(t-0))
$$

Пусть $\varrho_{1}, \ldots, \varrho_{k}-$ все различные нули $\zeta(s)$ на луче $\operatorname{Im} s=t, \operatorname{Re} s \geqslant 1 / 2$, и пусть $m_{1}, \ldots, m_{k}-$ их кратности. Тогда

$$
\frac{\zeta^{\prime}(s)}{\zeta(s)}=\sum_{\nu=1}^{k} \frac{m_{\nu}}{s-\varrho_{\nu}}+f(s)
$$

где функция $f$ аналитична в некоторой окрестности отрезка с концами в точках $1 / 2+i t$ и $2+i t$. Рассмотрим разность

$$
d(t)=F(t)-\frac{1}{2}(F(t+0)+F(t-0))
$$

Пользуясь определением $F(t)$, будем иметь:

$$
\begin{aligned}
d(t) & =\lim _{h \rightarrow+0}\left(F(t)-\frac{1}{2}(F(t+h)+F(t-h))\right) \\
& =-\lim _{h \rightarrow+0} \int_{1 / 2}^{+\infty} \operatorname{Im}\left(\frac{\zeta^{\prime}(\sigma+i t)}{\zeta(\sigma+i t)}\left(-\frac{1}{2}\left(\frac{\zeta^{\prime}(\sigma+i(t+h))}{\zeta(\sigma+i(t+h))}+\frac{\zeta^{\prime}(\sigma+i(t-h))}{\zeta(\sigma+i(t-h))}\right)\right) d \sigma\right.
\end{aligned}
$$




$$
\begin{aligned}
= & -\lim _{h \rightarrow+0} \int_{1 / 2}^{2} \operatorname{Im} \sum_{\nu=1}^{k} m_{\nu}\left(\frac{1}{\sigma-\beta_{\nu}}-\frac{1}{2}\left(\frac{1}{\sigma-\beta_{\nu}+i h}+\frac{1}{\sigma-\beta_{\nu}-i h}\right)\right) d \sigma \\
& -\lim _{h \rightarrow+0} \int_{1 / 2}^{2} \operatorname{Im}\left(f(\sigma+i t)-\frac{1}{2}(f(\sigma+i(t+h))+f(\sigma+i(t-h)))\right) d \sigma \\
& -\lim _{h \rightarrow+0} \int_{2}^{+\infty} \sum_{n=2}^{+\infty} \frac{\Lambda(n)}{n^{\sigma+i t}}\left(\frac{n^{i h}+n^{-i h}}{2}-1\right) d \sigma \\
= & \lim _{h \rightarrow+0} \int_{1 / 2}^{2} \sum_{\nu=1}^{k} \frac{m_{\nu}}{2}\left(\frac{-h}{\left(\sigma-\beta_{\nu}\right)^{2}+h^{2}}+\frac{h}{\left(\sigma-\beta_{\nu}\right)^{2}+h^{2}}\right) d \sigma \\
& -\lim _{h \rightarrow+0} \int_{1 / 2}^{2} O\left(h^{2}\right) d \sigma+\lim _{h \rightarrow+0} \sum_{n=2}^{+\infty} \frac{\Lambda(n)}{n^{2+i t}}(1-\cos (h \ln n)) \\
= & 2 \lim _{h \rightarrow+0} \sum_{n=2}^{+\infty} \frac{\Lambda(n) \sin ^{2}\left(\frac{h}{2} \ln n\right)}{n^{2}}=0 .
\end{aligned}
$$

Лемма доказана.

ОПРЕДЕЛЕНИЕ 1 . Пусть $x \geqslant 2, n$-натуральное число. Определим функцию $\Lambda_{x}(n)$ равенствами:

$$
\Lambda_{x}(n)= \begin{cases}\Lambda(n), & \text { если } 1 \leqslant n \leqslant x, \\ \Lambda(n) \frac{\ln ^{2}\left(x^{3} / n\right)-2 \ln ^{2}\left(x^{2} / n\right)}{2 \ln ^{2} x}, & \text { если } x<n \leqslant x^{2}, \\ \Lambda(n) \frac{\ln ^{2}\left(x^{3} / n\right)}{2 \ln ^{2} x}, & \text { если } x^{2}<n \leqslant x^{3} .\end{cases}
$$

Лемма 5. Пусть $x \geqslant 10, s=\sigma+i t, \operatorname{Re} s>0, s \neq 1, \zeta(s) \neq 0$. Тогда справедлива формула:

$$
\begin{gathered}
\frac{\zeta^{\prime}(s)}{\zeta(s)}=-\sum_{n \leqslant x^{3}} \frac{\Lambda_{x}(n)}{n^{s}} \\
+\frac{1}{\ln ^{2} x}\left(\frac{x^{1-s}\left(1-x^{1-s}\right)^{2}}{(1-s)^{3}}+\sum_{n=1}^{+\infty} \frac{x^{-(2 n+s)}\left(1-x^{-(2 n+s)}\right)^{2}}{(2 n+s)^{3}}-\sum_{\varrho} \frac{x^{\varrho-s}\left(1-x^{\varrho-s}\right)^{2}}{(\varrho-s)^{3}}\right) .
\end{gathered}
$$

ДокАЗАТЕльство. Пусть $m$ - целое, $m \geqslant 2|t|+10, T_{m}=2 m+1$. Пользуясь следствием леммы 3 , выберем число $T_{m}^{\prime}$ так, чтобы выполнялись неравенства $4 m+$ $2 \leqslant T_{m}^{\prime}<4 m+3$ и для некоторой постоянной $c$ ордината любого комплексного нуля $\zeta(s)$ отстояла от $T_{m}^{\prime}$ не меньше чем на $c\left(\ln T_{m}\right)^{-1}$. Положим $\alpha=\sigma+2$ и обозначим буквой Г границу прямоугольника с вершинами в точках $\alpha \pm i T_{m}^{\prime},-T_{m} \pm i T_{m}^{\prime}$, обходимую против часовой стрелки. Наконец, обозначим символом $I$ следуюший интеграл по контуру $\Gamma$ :

$$
I=\frac{1}{2 \pi i} \int_{\Gamma} \frac{x^{z-s}\left(1-x^{z-s}\right)^{2}}{(z-s)^{3}} \frac{\zeta^{\prime}(z)}{\zeta(z)} d z .
$$


Подьнтегральная функция не имеет особенностей внутри и на границе рассматриваемого прямоугольника, за исключением полюсов в точках $z=s$ с вычетом $\zeta^{\prime}(s)(\ln x)^{2} / \zeta(s), z=1$ с вычетом $-x^{1-s}\left(1-x^{1-s}\right)^{2} /(1-s)^{3}, z=\varrho=\beta+i \gamma($ комплексные нули $\zeta(z)$ с $\left.|\gamma|<T_{m}^{\prime}\right)$ с вычетами $x^{\varrho-s}\left(1-x^{\varrho-s}\right)^{2} /(\varrho-s)^{3}$ и $z=-2 n$ (вещественные нули $\zeta(z)$ с $n=1,2, \ldots, m)$ с вычетами $x^{-(2 n+s)}\left(1-x^{-(2 n+s)}\right)^{2} /(2 n+s)^{3}$. По теореме Коши интеграл $I$ равен сумме этих вычетов. С другой стороны, $I=\sum_{k=1}^{4} I_{k}$, где $I_{k}, k=1,2,3,4,-$ интегралы по сторонам контура $\Gamma$.

Интегралы $I_{2}$ и $I_{4}$ по верхней и нижней сторонам прямоугольника оцениваются одинаково. Оценим, например, $I_{2}$. Для этого представим его суммою

$$
I_{2}^{\prime}+I_{2}^{\prime \prime}=-\left(\int_{-T_{m}+i T_{m}^{\prime}}^{1 / 2+i T_{m}^{\prime}}+\int_{1 / 2+i T_{m}^{\prime}}^{\alpha+i T_{m}^{\prime}}\right)
$$

Тогда

$$
\begin{aligned}
\left|I_{2}^{\prime}\right| & =\frac{1}{2 \pi}\left|\int_{-T_{m}}^{1 / 2} \frac{x^{u-\sigma+i\left(T_{m}^{\prime}-t\right)}\left(1-x^{u-\sigma+i\left(T_{m}^{\prime}-t\right)}\right)^{2}}{\left(u-\sigma+i\left(T_{m}^{\prime}-t\right)\right)^{3}} \frac{\zeta^{\prime}\left(u+i T_{m}^{\prime}\right)}{\zeta\left(u+i T_{m}^{\prime}\right)} d u\right| \\
& \leqslant \frac{x^{1 / 2-\sigma}\left(1+x^{1 / 2-\sigma}\right)^{2}}{T_{m}^{3}} \int_{-T_{m}}^{1 / 2}\left|\frac{\zeta^{\prime}\left(u+i T_{m}^{\prime}\right)}{\zeta\left(u+i T_{m}^{\prime}\right)}\right| d u \leqslant \frac{4 x^{3 / 2}}{T_{m}^{3}} \int_{-T_{m}}^{1 / 2}\left|\frac{\zeta^{\prime}\left(u+i T_{m}^{\prime}\right)}{\zeta\left(u+i T_{m}^{\prime}\right)}\right| d u .
\end{aligned}
$$

Чтобы оценить подынтегральное выражение, воспользуемся равенством

$$
\frac{\zeta^{\prime}\left(u+i T_{m}^{\prime}\right)}{\zeta\left(u+i T_{m}^{\prime}\right)}=-\frac{1}{2}\left(\frac{\Gamma^{\prime}}{\Gamma}\left(\frac{u+i T_{m}^{\prime}}{2}\right)+\frac{\Gamma^{\prime}}{\Gamma}\left(\frac{1-u-i T_{m}^{\prime}}{2}\right)\right)-\frac{\zeta^{\prime}\left(1-u-i T_{m}^{\prime}\right)}{\zeta\left(1-u-i T_{m}^{\prime}\right)}+\ln \pi,
$$

которое следует из функционального уравнения для $\xi$-функции. Заметим, что для рассматриваемых значений $u$

$$
\begin{gathered}
\left|\arg \left(\frac{u+i T_{m}^{\prime}}{2}\right)\right| \leqslant \pi-\operatorname{arctg} \frac{T_{m}^{\prime}}{T_{m}} \leqslant \pi-\operatorname{arctg} 2 \leqslant \frac{3}{4} \pi, \\
\left|\arg \left(\frac{1-u-i T_{m}^{\prime}}{2}\right)\right| \leqslant \frac{\pi}{2}-\operatorname{arctg} \frac{1}{2 T_{m}^{\prime}} \leqslant \frac{\pi}{2} .
\end{gathered}
$$

Так как для комплексных $z$ с условием $|\arg z| \leqslant 3 \pi / 4$ справедлива асимптотика

$$
\frac{\Gamma^{\prime}(z)}{\Gamma(z)}=\ln z+O\left(|z|^{-1}\right)
$$

то первые два слагаемых в выражении для $\left(\zeta^{\prime} / \zeta\right)\left(u+i T_{m}^{\prime}\right)$ являются величинами порядка $\ln T_{m}$. Полагая для краткости $w=1-u-i T_{m}^{\prime}$ (так что $\left.\operatorname{Re} w \geqslant 1 / 2\right)$ и замечая, что при $1 / 2 \leqslant \operatorname{Re} w \leqslant 2$

$$
\frac{\zeta^{\prime}(w)}{\zeta(w)}=\sum_{\left|\gamma+T_{m}^{\prime}\right| \leqslant 1}(w-\varrho)^{-1}+O\left(\ln T_{m}\right)=\sum_{\left|\gamma+T_{m}^{\prime}\right| \leqslant 1} O\left(\ln T_{m}\right)+O\left(\ln T_{m}\right)=O\left(\ln ^{2} T_{m}\right)
$$


а при $\operatorname{Re} w \geqslant 2\left(\zeta^{\prime} / \zeta\right)(w)=O(1)$, убеждаемся, что вклад от двух последних слагаемых в равенстве для логарифмической производной дзеты есть $O\left(\ln ^{2} T_{m}\right)$. Таким образом,

$$
I_{2}^{\prime}=O\left(\frac{x^{3 / 2}}{T_{m}^{3}} T_{m} \ln ^{2} T_{m}\right)=O\left(\frac{x^{3 / 2}}{T_{m}}\right)
$$

Для оценки $I_{2}^{\prime \prime}$ достаточно воспользоваться равенством

$$
\frac{\zeta^{\prime}\left(u+i T_{m}^{\prime}\right)}{\zeta\left(u+i T_{m}^{\prime}\right)}=\sum_{\left|\gamma-T_{m}^{\prime}\right| \leqslant 1}\left(u+i T_{m}^{\prime}-\varrho\right)^{-1}+O\left(\ln T_{m}\right)=O\left(\ln ^{2} T_{m}\right)
$$

Таким образом находим:

$$
I_{2}^{\prime \prime}=O\left(\frac{x^{3 / 2}}{T_{m}^{3}} \ln ^{2} T_{m}\right)=O\left(\frac{x^{3 / 2}}{T_{m}}\right), \quad I_{2}, I_{4}=O\left(\frac{x^{3 / 2}}{T_{m}}\right)
$$

Для того чтобы оценить интеграл $I_{3}$ по левой стороне контура, положим $z=-T_{m}+i u$ и снова воспользуемся функциональным уравнением для $\xi(s)$ :

$$
\begin{aligned}
\left|I_{3}\right| \leqslant & \frac{4 x^{-(2 m+1+\sigma)}}{T_{m}^{3}} \int_{-T_{m}^{\prime}}^{T_{m}^{\prime}}\left|\frac{\zeta^{\prime}\left(-T_{m}+i u\right)}{\zeta\left(-T_{m}+i u\right)}\right| d u \\
\leqslant & \frac{x^{-2 m}}{T_{m}^{3}} \int_{-T_{m}^{\prime}}^{T_{m}^{\prime}}\left(\left|\frac{\Gamma^{\prime}}{\Gamma}\left(\frac{-T_{m}^{\prime}+i u}{2}\right)\right|+\left|\frac{\Gamma^{\prime}}{\Gamma}\left(\frac{1+T_{m}^{\prime}-i u}{2}\right)\right|\right) d u \\
& +\frac{x^{-2 m}}{T_{m}^{3}} \int_{-T_{m}^{\prime}}^{T_{m}^{\prime}}\left(\left|\frac{\zeta^{\prime}\left(1+T_{m}-i u\right)}{\zeta\left(1+T_{m}-i u\right)}\right|+\ln \pi\right) d u
\end{aligned}
$$

Поскольку $\operatorname{Re}\left(-\frac{T_{m}+i u}{2}\right)=-(m+1 / 2)$, то по лемме 1 на отрезке $\left[-T_{m}^{\prime}, T_{m}^{\prime}\right]$ первое слагаемое есть $O\left(\ln T_{m}\right)$. Так как

$$
\left|\arg \left(\frac{1+T_{m}-i u}{2}\right)\right| \leqslant \frac{\pi}{2}
$$

то второе слагаемое по порядку величины также не превосходит $\ln T_{m}$. Наконец, два последних слагаемых не превосходят абсолютной постоянной. Следовательно,

$$
I_{3}=O\left(\frac{x^{-2 m}}{T_{m}^{2}} \ln T_{m}\right)=O\left(\frac{x^{-2 m}}{T_{m}}\right)
$$

Устремим теперь $m$ к бесконечности. Тогда $I_{2}, I_{3}, I_{4} \rightarrow 0$ и мы приходим к равенству

$$
\begin{gathered}
\frac{1}{2 \pi i} \int_{\alpha-i \infty}^{\alpha+\infty} \frac{x^{z-s}\left(1-x^{z-s}\right)^{2}}{(z-s)^{3}} \frac{\zeta^{\prime}(z)}{\zeta(z)} d z=\frac{\zeta^{\prime}(s)}{\zeta(s)} \ln ^{2} x-\frac{x^{1-s}\left(1-x^{1-s}\right)^{2}}{(1-s)^{3}} \\
\quad+\sum_{\varrho} \frac{x^{\varrho-s}\left(1-x^{\varrho-s}\right)^{2}}{(\varrho-s)^{3}}-\sum_{n=1}^{+\infty} \frac{x^{-(2 n+s)}\left(1-x^{-(2 n+s)}\right)^{2}}{(2 n+s)^{3}}
\end{gathered}
$$


На прямой интегрирования $\operatorname{Re} z=\alpha$ разложим $\left(\zeta^{\prime} / \zeta\right)(z)$ в абсолютно сходящийся ряд Дирихле и поменяем местами порядки суммирования и интегрирования. Тогда левая часть последнего равенства примет вид

$$
\begin{aligned}
- & \sum_{n=1}^{+\infty} \frac{\Lambda(n)}{n^{s}} \frac{1}{2 \pi i} \int_{\alpha-i \infty}^{\alpha+\infty} \frac{x^{z-s}\left(1-x^{z-s}\right)^{2}}{(z-s)^{3}} \frac{d z}{n^{z-s}} \\
= & -\sum_{n=1}^{+\infty} \frac{\Lambda(n)}{n^{s}} \frac{1}{2 \pi i} \int_{2-i \infty}^{2+\infty}\left(\left(\frac{x}{n}\right)^{w}-2\left(\frac{x^{2}}{n}\right)^{w}+\left(\frac{x^{3}}{n}\right)^{w}\right) \frac{d w}{w^{3}} \\
= & -\sum_{n \leqslant x} \frac{\Lambda(n)}{n^{s}}\left(\frac{1}{2} \ln ^{2} \frac{x}{n}-\ln ^{2} \frac{x^{2}}{n}+\frac{1}{2} \ln ^{2} \frac{x^{3}}{n}\right) \\
& -\sum_{x<n \leqslant x^{2}} \frac{\Lambda(n)}{n^{s}}\left(\frac{1}{2} \ln ^{2} \frac{x^{3}}{n}-\ln ^{2} \frac{x^{2}}{n}\right)-\sum_{x^{2}<n \leqslant x^{3}} \frac{\Lambda(n)}{n^{s}} \frac{1}{2} \ln ^{2} \frac{x^{3}}{n} \\
= & -\ln ^{2} x \sum_{n \leqslant x^{3}} \frac{\Lambda_{x}(n)}{n^{s}} .
\end{aligned}
$$

Отсюда следует утверждение леммы.

ОПРЕДЕЛЕНИЕ 2 . Пусть $2 \leqslant x \leqslant t^{2}$. Положим

$$
\sigma_{x, t}=\frac{1}{2}+2 \max \left(\left|\beta-\frac{1}{2}\right|, \frac{1}{\ln x}\right),
$$

где $\beta$ пробегает вещественные части нулей $\varrho=\beta+i \gamma$ дзета-функции Римана, удовлетворяюшие условию

$$
|t-\gamma| \leqslant \frac{x^{3|\beta-1 / 2|}}{\ln x}
$$

Символом $r(x, t)$ будем обозначать сумму

$$
\sum_{n \leqslant x^{3}} \frac{\Lambda_{x}(n)}{n^{\sigma_{x, t}+i t}}
$$

ЛЕмма 6. При $2 \leqslant x \leqslant t^{2}$ справедливо неравенство:

$$
\sum_{\varrho} \frac{1}{\left(\sigma_{x, t}-\beta\right)^{2}+(t-\gamma)^{2}} \leqslant \frac{5}{3}\left(\sigma_{x, t}-\frac{1}{2}\right)^{-1}\left(\operatorname{Re} \frac{\zeta^{\prime}\left(\sigma_{x, t}+i t\right)}{\zeta\left(\sigma_{x, t}+i t\right)}+\frac{1}{2} \ln t+3\right) .
$$

ДокАЗАТЕльство. Полагая в лемме $2 s=\sigma_{x, t}+i t$ и отделяя в получившемся равенстве вещественную часть, получим:

$$
\operatorname{Re} \frac{\zeta^{\prime}\left(\sigma_{x, t}+i t\right)}{\zeta\left(\sigma_{x, t}+i t\right)}=\sum_{\varrho} \frac{\sigma_{x, t}-\beta}{\left(\sigma_{x, t}-\beta\right)^{2}+(t-\gamma)^{2}}+\theta\left(\frac{1}{2} \ln t+3\right)
$$


Воспользовавшись симметрией комплексных нулей дзета-функции, преобразуем это соотношение к виду

$$
\begin{aligned}
\operatorname{Re} \frac{\zeta^{\prime}\left(\sigma_{x, t}+i t\right)}{\zeta\left(\sigma_{x, t}+i t\right)}= & \frac{1}{2} \sum_{\varrho}\left(\frac{\sigma_{x, t}-\beta}{\left(\sigma_{x, t}-\beta\right)^{2}+(t-\gamma)^{2}}+\frac{\sigma_{x, t}-1+\beta}{\left(\sigma_{x, t}-1+\beta\right)^{2}+(t-\gamma)^{2}}\right) \\
& +\theta\left(\frac{1}{2} \ln t+3\right) \\
= & \left(\sigma_{x, t}-\frac{1}{2}\right) \sum_{\varrho} \frac{\left(\sigma_{x, t}-\frac{1}{2}\right)^{2}-\left(\beta-\frac{1}{2}\right)^{2}+(t-\gamma)^{2}}{\left(\left(\sigma_{x, t}-\beta\right)^{2}+(t-\gamma)^{2}\right)\left(\left(\sigma_{x, t}-1+\beta\right)^{2}+(t-\gamma)^{2}\right)} \\
& +\theta\left(\frac{1}{2} \ln t+3\right) .
\end{aligned}
$$

Обозначим числитель дроби, стоящей под знаком суммы, символом $\varkappa(\varrho)$ и покажем, что

$$
\varkappa(\varrho) \geqslant 0.3\left(\left(\sigma_{x, t}-\beta\right)^{2}+\left(\sigma_{x, t}-1+\beta\right)^{2}+2(t-\gamma)^{2}\right) .
$$

Для этого рассмотрим два случая. Пусть сначала $|\beta-1 / 2| \leqslant \frac{1}{2}\left(\sigma_{x, t}-1 / 2\right)$. Тогда

$$
\begin{aligned}
\varkappa(\varrho) & \geqslant\left(\sigma_{x, t}-\frac{1}{2}\right)^{2}-\frac{1}{4}\left(\sigma_{x, t}-\frac{1}{2}\right)^{2}+(t-\gamma)^{2} \\
& =\frac{3}{5}\left(\sigma_{x, t}-\frac{1}{2}\right)^{2}+\frac{3}{20}\left(\sigma_{x, t}-\frac{1}{2}\right)^{2}+(t-\gamma)^{2} \\
& \geqslant \frac{3}{5}\left(\sigma_{x, t}-\frac{1}{2}\right)^{2}+\frac{3}{5}\left(\beta-\frac{1}{2}\right)^{2}+(t-\gamma)^{2} \\
& =\frac{3}{10}\left(2 \sigma_{x, t}^{2}-2 \sigma_{x, t}+2 \beta^{2}-2 \beta+1\right)+(t-\gamma)^{2} \\
& =\frac{3}{10}\left(\left(\sigma_{x, t}-\beta\right)^{2}+\left(\sigma_{x, t}-1+\beta\right)^{2}+\frac{10}{3}(t-\gamma)^{2}\right) \\
& \geqslant 0.3\left(\left(\sigma_{x, t}-\beta\right)^{2}+\left(\sigma_{x, t}-1+\beta\right)^{2}+2(t-\gamma)^{2}\right) .
\end{aligned}
$$

Пусть теперь $|\beta-1 / 2|>\frac{1}{2}\left(\sigma_{x, t}-1 / 2\right)$, т.е. $\sigma_{x, t}<1 / 2+2|\beta-1 / 2|$. Из последнего неравенства и определения $\sigma_{x, t}$ следует, что

и

$$
|t-\gamma|>\frac{x^{3|\beta-1 / 2|}}{\ln x}>3\left|\beta-\frac{1}{2}\right|
$$

$$
(t-\gamma)^{2}=\frac{2}{3}(t-\gamma)^{2}+\frac{1}{3}(t-\gamma)^{2}>\frac{2}{3}(t-\gamma)^{2}+3\left(\beta-\frac{1}{2}\right)^{2}
$$

Таким образом,

$$
\begin{aligned}
\varkappa(\varrho) & >\left(\sigma_{x, t}-\frac{1}{2}\right)^{2}+2\left(\beta-\frac{1}{2}\right)^{2}+\frac{2}{3}(t-\gamma)^{2} \\
& \geqslant \frac{2}{3}\left(\left(\sigma_{x, t}-\frac{1}{2}\right)^{2}+\left(\beta-\frac{1}{2}\right)^{2}+(t-\gamma)^{2}\right) \\
& \geqslant 0.3\left(2\left(\sigma_{x, t}-\frac{1}{2}\right)^{2}+2\left(\beta-\frac{1}{2}\right)^{2}+2(t-\gamma)^{2}\right) \\
& =0.3\left(\left(\sigma_{x, t}-\beta\right)^{2}+\left(\sigma_{x, t}-1+\beta\right)^{2}+2(t-\gamma)^{2}\right) .
\end{aligned}
$$


Возврашаясь к равенству для $\left(\zeta^{\prime} / \zeta\right)\left(\sigma_{x, t}+i t\right)$, находим:

$$
\begin{aligned}
\operatorname{Re} \frac{\zeta^{\prime}\left(\sigma_{x, t}+i t\right)}{\zeta\left(\sigma_{x, t}+i t\right)} \\
=0.3\left(\sigma_{x, t}-\frac{1}{2}\right) \sum_{\varrho} \frac{\left(\sigma_{x, t}-\beta\right)^{2}+\left(\sigma_{x, t}-1+\beta\right)^{2}+2(t-\gamma)^{2}}{\left(\left(\sigma_{x, t}-\beta\right)^{2}+(t-\gamma)^{2}\right)\left(\left(\sigma_{x, t}-1+\beta\right)^{2}+(t-\gamma)^{2}\right)} \\
\quad+\theta\left(\frac{1}{2} \ln t+3\right) \\
=0.3\left(\sigma_{x, t}-\frac{1}{2}\right) \sum_{\varrho}\left(\frac{1}{\left(\sigma_{x, t}-\beta\right)^{2}+(t-\gamma)^{2}}+\frac{1}{\left(\sigma_{x, t}-1+\beta\right)^{2}+(t-\gamma)^{2}}\right) \\
\quad+\theta\left(\frac{1}{2} \ln t+3\right) \\
=\frac{3}{5}\left(\sigma_{x, t}-\frac{1}{2}\right) \sum_{\varrho} \frac{1}{\left(\sigma_{x, t}-\beta\right)^{2}+(t-\gamma)^{2}}+\theta\left(\frac{1}{2} \ln t+3\right),
\end{aligned}
$$

так что

$$
\sum_{\varrho} \frac{1}{\left(\sigma_{x, t}-\beta\right)^{2}+(t-\gamma)^{2}} \leqslant \frac{5}{3}\left(\sigma_{x, t}-\frac{1}{2}\right)^{-1}\left(\operatorname{Re} \frac{\zeta^{\prime}\left(\sigma_{x, t}+i t\right)}{\zeta\left(\sigma_{x, t}+i t\right)}+\frac{1}{2} \ln t+3\right) .
$$

Лемма доказана.

Лемма 7. Пусть $s=\sigma+i t, 10 \leqslant x \leqslant t^{2}, \sigma \geqslant \sigma_{x, t}$. Тогдa

$$
\frac{\zeta^{\prime}(s)}{\zeta(s)}+\sum_{n \leqslant x^{3}} \frac{\Lambda_{x}(n)}{n^{s}}=\frac{13 \theta}{3} x^{\frac{1}{2}-\frac{\sigma}{2}}\left(|r(x, t)|+\frac{1}{2} \ln t+4\right) .
$$

ДоКАЗАТЕЛЬСТво. Согласно лемме 5 , левая часть последнего равенства может быть записана в виде $\left(R_{1}+R_{2}-R_{3}\right)(\ln x)^{-2}$, где

$$
\begin{aligned}
R_{1} & =\frac{x^{1-s}\left(1-x^{1-s}\right)^{2}}{(1-s)^{3}} \\
R_{2} & =\sum_{n=1}^{+\infty} \frac{x^{-(2 n+s)}\left(1-x^{-(2 n+s)}\right)^{2}}{(2 n+s)^{3}} \\
R_{3} & =\sum_{\varrho} \frac{x^{\varrho-s}\left(1-x^{\varrho-s}\right)^{2}}{(\varrho-s)^{3}} .
\end{aligned}
$$

Сумма $R_{1}+R_{2}$ по абсолютной величине не превосходит

$$
\begin{gathered}
t^{-3}\left(x^{1-\sigma}\left(1-x^{1-\sigma}\right)^{2}+\sum_{n=1}^{+\infty} x^{-(2 n+\sigma)}\left(1+x^{-(2 n+\sigma)}\right)^{2}\right) \\
\quad<2 x^{-3 / 2}\left(x^{1-\sigma}+x^{3(1-\sigma)}+x^{-\sigma} \sum_{n=1}^{+\infty} x^{-2 n}\right) \\
\quad=2 x^{\frac{1}{4}-\frac{\sigma}{2}}\left(x^{-\frac{3+\sigma}{2}}+x^{\frac{5}{2}\left(\frac{1}{2}-\sigma\right)}+\frac{x^{-\frac{7}{2}-\frac{\sigma}{2}}}{x^{2}-1}\right) \\
\quad<2 x^{\frac{1}{4}-\frac{\sigma}{2}}\left(x^{-2}+e^{-5}+x^{-3}\right)<0.02 x^{\frac{1}{4}-\frac{\sigma}{2}} .
\end{gathered}
$$


Оценивая $R_{3}$ по модулю, будем иметь:

$$
\left|R_{3}\right| \leqslant \sum_{\varrho} \varkappa_{1}(\varrho), \quad \text { где } \varkappa_{1}(\varrho)=\varkappa_{1}(\beta+i \gamma)=\frac{x^{\beta-\sigma}\left(1+x^{\beta-\sigma}\right)^{2}}{\left((\sigma-\beta)^{2}+(t-\gamma)^{2}\right)^{3 / 2}} .
$$

Покажем, что оценка суммы по нулям дзета-функции сводится к оценке суммы из предыдущей леммы. Для этого рассмотрим два случая.

Пусть сначала $\beta \leqslant \frac{1}{2}\left(\sigma_{x, t}+1 / 2\right)$. Тогда

$$
\beta-\sigma \leqslant \frac{1}{2}\left(\sigma_{x, t}+\frac{1}{2}\right)-\sigma=\left(\frac{1}{4}-\frac{\sigma}{2}\right)-\frac{1}{2}\left(\sigma-\sigma_{x, t}\right) \leqslant \frac{1}{4}-\frac{\sigma}{2} .
$$

Кроме того, из условия $\sigma_{x, t} \geqslant 1 / 2+2(\ln x)^{-1}$ следует, что

$$
\beta-\sigma \leqslant \frac{\sigma_{x, t}}{2}+\frac{1}{4}-\sigma_{x, t} \leqslant-\frac{1}{\ln x}
$$

В этом случае

$$
\varkappa_{1}(\varrho) \leqslant \frac{x^{1 / 4-\sigma / 2}\left(1+x^{-1 / \ln x}\right)^{2}}{(\sigma-\beta)\left(\left(\sigma_{x, t}-\beta\right)^{2}+(t-\gamma)^{2}\right)} \leqslant\left(1+e^{-1}\right)^{2} \frac{x^{1 / 4-\sigma / 2} \ln x}{\left(\sigma_{x, t}-\beta\right)^{2}+(t-\gamma)^{2}} .
$$

Пусть теперь $\beta>\frac{1}{2}\left(\sigma_{x, t}+1 / 2\right)$. Тогда $\sigma_{x, t}<1 / 2+2(\beta-1 / 2)$. Из этого равенства и определения $\sigma_{x, t}$ заключаем, что

$$
|t-\gamma|>\frac{x^{3|\beta-1 / 2|}}{\ln x}>3\left|\beta-\frac{1}{2}\right|
$$

Поскольку $\sigma_{x, t}>1 / 2$, то $\beta-1 / 2>\beta-\sigma_{x, t}$. Кроме того,

$$
\beta-\frac{1}{2}=\left(\sigma_{x, t}-\beta\right)+2\left(\beta-\frac{1}{2}\left(\sigma_{x, t}+\frac{1}{2}\right)\right)>\sigma_{x, t}-\beta .
$$

Следовательно, $\beta-1 / 2>\left|\beta-\sigma_{x, t}\right|$ и $|t-\gamma|>3(\beta-1 / 2)>3\left|\beta-\sigma_{x, t}\right|$. Поэтому

$$
(t-\gamma)^{2}=\frac{8}{9}(t-\gamma)^{2}+\frac{1}{9}(t-\gamma)^{2}>\frac{8}{9}(t-\gamma)^{2}+\left(\beta-\sigma_{x, t}\right)^{2} \geqslant \frac{8}{9}\left((t-\gamma)^{2}+\left(\beta-\sigma_{x, t}\right)^{2}\right)
$$

и

$$
\begin{aligned}
\left((\sigma-\beta)^{2}+(t-\gamma)^{2}\right)^{3 / 2} & \geqslant|t-\gamma|\left((\sigma-\beta)^{2}+(t-\gamma)^{2}\right) \\
& >\frac{8}{9} \frac{x^{3(\beta-1 / 2)}}{\ln x}\left((t-\gamma)^{2}+\left(\beta-\sigma_{x, t}\right)^{2}\right)
\end{aligned}
$$

Возвращаясь к оценке $\varkappa_{1}(\varrho)$, получим:

$$
\varkappa_{1}(\varrho)<\frac{x^{\beta-\sigma}\left(1+x^{\beta-\sigma}\right)^{2}}{\frac{8}{9}(\ln x)^{-1}\left((t-\gamma)^{2}+\left(\beta-\sigma_{x, t}\right)^{2}\right)} \leqslant \frac{9}{8} \ln x \frac{x^{-2 \beta-\sigma+3 / 2}\left(1+x^{\beta-1 / 2}\right)^{2}}{(t-\gamma)^{2}+\left(\beta-\sigma_{x, t}\right)^{2}} .
$$


Числитель последней дроби не превосходит

$$
x^{\frac{1}{4}-\frac{\sigma}{2}} x^{\frac{5}{4}-\frac{\sigma}{2}-2 \beta}\left(1+x^{\beta-\frac{1}{2}}\right)^{2} \leqslant x^{\frac{1}{4}-\frac{\sigma}{2}} x^{1-2 \beta}\left(1+x^{\beta-\frac{1}{2}}\right)^{2} \leqslant x^{\frac{1}{4}-\frac{\sigma}{2}}\left(1+x^{\frac{1}{2}-\beta}\right)^{2} .
$$

Так как

$$
\frac{1}{2}-\beta \leqslant \frac{1}{2}-\frac{1}{2}\left(\sigma_{x, t}+\frac{1}{2}\right)=-\frac{1}{2}\left(\sigma_{x, t}-\frac{1}{2}\right) \leqslant-\frac{1}{\ln x}
$$

то

$$
\varkappa_{1}(\varrho) \leqslant \frac{9}{8}\left(1+e^{-1}\right)^{2} \frac{x^{\frac{1}{4}-\frac{\sigma}{2}} \ln x}{(t-\gamma)^{2}+\left(\beta-\sigma_{x, t}\right)^{2}} .
$$

Пользуясь оценкой предыдушей леммы, мы приходим к следующему соотношению:

$$
\begin{aligned}
& \frac{\zeta^{\prime}(\sigma+i t)}{\zeta(\sigma+i t)}+\sum_{n \leqslant x^{3}} \frac{\Lambda_{x}(n)}{n^{\sigma+i t}}=\frac{2 \theta}{\ln ^{2} x} x^{\frac{1}{4}-\frac{\sigma}{2}}\left(\sum_{\varrho} \frac{\ln x}{\left(\sigma_{x, t}-\beta\right)^{2}+(t-\gamma)^{2}}+0.01\right) \\
& =\frac{2 \theta x^{\frac{1}{4}-\frac{\sigma}{2}}}{\ln ^{2} x}\left(\frac{5}{3}\left(\sigma_{x, t}-\frac{1}{2}\right)^{-1}(\ln x)\left(\operatorname{Re} \frac{\zeta^{\prime}\left(\sigma_{x, t}+i t\right)}{\zeta\left(\sigma_{x, t}+i t\right)}+0.5 \ln |t|+3\right)+0.01\right) \\
& =\frac{5 \theta}{3} x^{\frac{1}{4}-\frac{\sigma}{2}}\left(\operatorname{Re} \frac{\zeta^{\prime}\left(\sigma_{x, t}+i t\right)}{\zeta\left(\sigma_{x, t}+i t\right)}+0.5 \ln |t|+3.01\right) .
\end{aligned}
$$

Положим в нем $\sigma=\sigma_{x, t}$. После преобразований получим:

$$
\frac{\zeta^{\prime}\left(\sigma_{x, t}+i t\right)}{\zeta\left(\sigma_{x, t}+i t\right)}\left(1-\frac{5 \theta_{1}}{3} x^{\frac{1}{4}-\frac{\sigma_{x, t}}{2}}\right)=-\sum_{n \leqslant x^{3}} \frac{\Lambda_{x}(n)}{n^{\sigma_{x, t}+i t}}+\frac{5 \theta_{2}}{3} x^{\frac{1}{4}-\frac{\sigma_{x, t}}{2}}\left(\frac{1}{2} \ln t+3.01\right) .
$$

Так как $1 / 4-\sigma_{x, t} / 2 \leqslant-(\ln x)^{-1}$, то

$$
\frac{\zeta^{\prime}\left(\sigma_{x, t}+i t\right)}{\zeta\left(\sigma_{x, t}+i t\right)}\left(1-\frac{5 \theta_{3}}{3 e}\right)=-r(x, t)+\frac{5 \theta_{4}}{3 e}(0.5 \ln t+3.01)
$$

и

$$
\begin{aligned}
\left|\operatorname{Re} \frac{\zeta^{\prime}\left(\sigma_{x, t}+i t\right)}{\zeta\left(\sigma_{x, t}+i t\right)}\right| & \leqslant\left|\frac{\zeta^{\prime}\left(\sigma_{x, t}+i t\right)}{\zeta\left(\sigma_{x, t}+i t\right)}\right| \leqslant \frac{1}{1-5 e^{-1} / 3}\left(|r(x, t)|+\frac{5}{3 e}(0.5 \ln t+3.01)\right) \\
& <2.6|r(x, t)|+1.6(0.5 \ln t+3.01) .
\end{aligned}
$$

Поэтому

$$
\begin{aligned}
\frac{\zeta^{\prime}(\sigma+i t)}{\zeta(\sigma+i t)}+\sum_{n \leqslant x^{3}} \frac{\Lambda_{x}(n)}{n^{\sigma+i t}} & =\frac{5 \theta_{5}}{3} x^{\frac{1}{4}-\frac{\sigma}{2}}(2.6|r(x, t)|+2.6(0.5 \ln t+3.01)) \\
& =\frac{13 \theta_{6}}{3} x^{\frac{1}{4}-\frac{\sigma}{2}}\left(|r(x, t)|+\frac{1}{2} \ln t+4\right)
\end{aligned}
$$

Лемма доказана. 
СлЕДСТВИЕ. При $10<x \leqslant t^{2}$ справедливы неравенства:

$$
\begin{gathered}
\left|\frac{\zeta^{\prime}\left(\sigma_{x, t}+i t\right)}{\zeta\left(\sigma_{x, t}+i t\right)}\right|<2.6|r(x, t)|+1.6(0.5 \ln |t|+3.01) \\
\sum_{\varrho} \frac{1}{\left(\sigma_{x, t}-\beta\right)^{2}+(t-\gamma)^{2}}<\frac{13}{3}\left(\sigma_{x, t}-\frac{1}{2}\right)^{-1}\left(|r(x, t)|+\frac{1}{2} \ln |t|+4\right) .
\end{gathered}
$$

Доказательство первого неравенства получено выше. Второе неравенство есть следствие первого и леммы 6.

Teоpema 1. Пусть $e^{16}<x \leqslant t^{2}$. Тогда

$$
S(t)=-\frac{1}{\pi} \sum_{n \leqslant x^{3}} \frac{\Lambda_{x}(n)}{n^{\sigma_{x, t}}} \frac{\sin (t \ln n)}{\ln n}+15 \theta\left(\sigma_{x, t}-\frac{1}{2}\right)(|r(x, t)|+\ln |t|) .
$$

ДОКАЗАТЕЛЬСТВО. Пользуясь формулой леммы 4, представим величину

$$
\pi S(t)=-\operatorname{Im} \int_{1 / 2}^{+\infty} \frac{\zeta^{\prime}(\sigma+i t)}{\zeta(\sigma+i t)} d \sigma
$$

в виде суммы $I_{1}+I_{2}+I_{3}$, в которой

$$
\begin{aligned}
& I_{1}=-\operatorname{Im} \int_{\sigma_{x, t}}^{+\infty} \frac{\zeta^{\prime}(\sigma+i t)}{\zeta(\sigma+i t)} d \sigma \\
& I_{2}=\left(\sigma_{x, t}-\frac{1}{2}\right) \operatorname{Im} \frac{\zeta^{\prime}\left(\sigma_{x, t}+i t\right)}{\zeta\left(\sigma_{x, t}+i t\right)} \\
& I_{3}=\operatorname{Im} \int_{1 / 2}^{\sigma_{x, t}}\left(\frac{\zeta^{\prime}\left(\sigma_{x, t}+i t\right)}{\zeta\left(\sigma_{x, t}+i t\right)}-\frac{\zeta^{\prime}(\sigma+i t)}{\zeta(\sigma+i t)}\right) d \sigma .
\end{aligned}
$$

Согласно лемме 7 и следствию из нее,

$$
\begin{aligned}
I_{1} & =-\operatorname{Im} \sum_{n \leqslant x^{3}} \frac{\Lambda_{x}(n)}{n^{i t}} \int_{\sigma_{x, t}}^{+\infty} n^{-\sigma} d \sigma+\frac{13 \theta}{3}(|r(x, t)|+0.5 \ln |t|+4) \int_{\sigma_{x, t}}^{+\infty} x^{\frac{1}{4}-\frac{\sigma}{2}} d \sigma \\
& =-\sum_{n \leqslant x^{3}} \frac{\Lambda_{x}(n)}{n^{\sigma_{x, t}}} \frac{\sin (t \ln n)}{\ln n}+\frac{13 \theta}{3 e}(|r(x, t)|+0.5 \ln |t|+4)\left(\sigma_{x, t}-\frac{1}{2}\right), \\
\left|I_{2}\right| & \leqslant\left(\sigma_{x, t}-\frac{1}{2}\right)\left|\frac{\zeta^{\prime}\left(\sigma_{x, t}+i t\right)}{\zeta\left(\sigma_{x, t}+i t\right)}\right|<\left(\sigma_{x, t}-\frac{1}{2}\right)(2.6|r(x, t)|+1.6(0.5 \ln t+4)) .
\end{aligned}
$$

Далее, по лемме 2

$$
\begin{aligned}
& \operatorname{Im}\left(\frac{\zeta^{\prime}\left(\sigma_{x, t}+i t\right)}{\zeta\left(\sigma_{x, t}+i t\right)}-\frac{\zeta^{\prime}(\sigma+i t)}{\zeta(\sigma+i t)}\right) \\
& \quad=\operatorname{Im} \sum_{\varrho}\left(\frac{1}{\sigma_{x, t}-\beta+i(t-\gamma)}-\frac{1}{\sigma-\beta+i(t-\gamma)}\right)+\theta(\ln |t|+6) \\
& \quad=\sum_{\varrho} \frac{(t-\gamma)\left(\sigma_{x, t}+\sigma-2 \beta\right)\left(\sigma_{x, t}-\sigma\right)}{\left(\left(\sigma_{x, t}-\beta\right)^{2}+(t-\gamma)^{2}\right)\left((\sigma-\beta)^{2}+(t-\gamma)^{2}\right)}+\theta(\ln |t|+6),
\end{aligned}
$$


так что

$\left|I_{3}\right| \leqslant \sum_{\varrho} \frac{\sigma_{x, t}-1 / 2}{\left(\sigma_{x, t}-\beta\right)^{2}+(t-\gamma)^{2}} \int_{1 / 2}^{\sigma_{x, t}} \frac{|t-\gamma|\left|\sigma_{x, t}+\sigma-2 \beta\right| d \sigma}{(\sigma-\beta)^{2}+(t-\gamma)^{2}}+\left(\sigma_{x, t}-\frac{1}{2}\right)(\ln |t|+6)$.

Если $|\beta-1 / 2| \leqslant 0.5\left(\sigma_{x, t}-1 / 2\right)$, то при $1 / 2 \leqslant \sigma \leqslant \sigma_{x, t}$ справедливо неравенство

$$
\left|\sigma_{x, t}+\sigma-2 \beta\right|=\left|\left(\sigma_{x, t}-\frac{1}{2}\right)+\left(\sigma-\frac{1}{2}\right)-2\left(\beta-\frac{1}{2}\right)\right| \leqslant 3\left(\sigma_{x, t}-\frac{1}{2}\right) .
$$

В этом случае интеграл по $\sigma$ не превосходит

$$
3\left(\sigma_{x, t}-\frac{1}{2}\right) \int_{-\infty}^{+\infty} \frac{|t-\gamma| d u}{u^{2}+(t-\gamma)^{2}}=3 \pi\left(\sigma_{x, t}-\frac{1}{2}\right)
$$

Если же $|\beta-1 / 2|>0.5\left(\sigma_{x, t}-1 / 2\right)$, то

$$
\begin{aligned}
|t-\gamma| & >\frac{x^{3|\beta-1 / 2|}}{\ln x}>3\left|\beta-\frac{1}{2}\right| \\
\left|\sigma_{x, t}+\sigma-2 \beta\right| & \leqslant\left|\sigma-\frac{1}{2}\right|+\left|\sigma_{x, t}-\frac{1}{2}\right|+2\left|\beta-\frac{1}{2}\right| \\
& \leqslant 2\left|\sigma_{x, t}-\frac{1}{2}\right|+2\left|\beta-\frac{1}{2}\right|<6\left|\beta-\frac{1}{2}\right|
\end{aligned}
$$

и рассматриваемьй интеграл не превзойдет

$$
\int_{1 / 2}^{\sigma_{x, t}} \frac{|t-\gamma| 6|\beta-1 / 2|}{(t-\gamma)^{2}} d \sigma \leqslant \int_{1 / 2}^{\sigma_{x, t}} \frac{6|\beta-1 / 2|}{3|\beta-1 / 2|} d \sigma=2\left(\sigma_{x, t}-\frac{1}{2}\right) .
$$

Таким образом,

$$
\begin{aligned}
\left|I_{3}\right| & \leqslant 3 \pi\left(\sigma_{x, t}-\frac{1}{2}\right) \sum_{\varrho} \frac{\sigma_{x, t}-1 / 2}{\left(\sigma_{x, t}-\beta\right)^{2}+(t-\gamma)^{2}}+\left(\sigma_{x, t}-\frac{1}{2}\right)(\ln |t|+6) \\
& <3 \pi\left(\sigma_{x, t}-\frac{1}{2}\right) \frac{13}{3}(|r(x, t)|+0.5 \ln |t|+4)+\left(\sigma_{x, t}-\frac{1}{2}\right)(\ln |t|+6) .
\end{aligned}
$$

Сложив полученные неравенства, будем иметь:

$$
\left|S(t)+\frac{1}{\pi} \sum_{n \leqslant x^{3}} \frac{\Lambda_{x}(n)}{n^{\sigma_{x, t}}} \frac{\sin (t \ln n)}{\ln n}\right|<\frac{1}{\pi}\left(\sigma_{x, t}-\frac{1}{2}\right)\left(c_{1}|r(x, t)|+c_{2} \ln |t|+c_{3}\right),
$$

где

$$
c_{1}=\frac{13}{3 e}+2.6+13 \pi, \quad c_{2}=\frac{13}{6 e}+0.8+\frac{13 \pi}{2}+1, \quad c_{3}=\frac{52}{3 e}+6.4+52 \pi+6 .
$$

Для завершения доказательства остается заметить, что $c_{1}<15 \pi$ и при $|t|>e^{8}$ имеем $c_{2} \ln |t|+c_{3}<15 \pi \ln |t|$. Теорема доказана. 
Лемма 8. Имеет место формула:

$$
S_{1}(T)+\frac{1}{\pi} \mathrm{v} \cdot \mathrm{p} \cdot \int_{1 / 2}^{+\infty} \ln |\zeta(\sigma)| d \sigma-\frac{1}{\pi} \mathrm{v} \cdot \mathrm{p} \cdot \int_{1 / 2}^{+\infty} \ln |\zeta(\sigma+i T)| d \sigma=0 .
$$

ДокАЗАТЕЛЬСТво. Так как $S_{1}(T)$ - четная функция, принимающая в точке $T=0$ нулевое значение, то достаточно рассмотреть лиш случай $T>0$. Пусть $A \geqslant 10$. Обозначим буквой $\Gamma$ проходимый против часовой стрелки контур прямоугольника с вершинами в точках $1 / 2, A, A+i T, 1 / 2+i T$, в котором к каждому из нулей дзета-функции $\varrho=\beta+i \gamma \mathrm{c} \beta>1 / 2,0<\gamma<T$ от критической прямой проведен разрез, перпендикулярньй мнимой оси. Если $T$ совпадает с ординатой одного из нулей $\zeta(s)$, то верхнюю сторону прямоугольника $Г$ будем считать идущей вдоль нижнего берега разреза.

Так как функция $\ln \zeta(s)$ аналитична внутри построенного контура и имеет в точке $s=1$ логарифмическую особенность с нулевьм вычетом, то по теореме Коши имеем равенство:

$$
\operatorname{Re} \int_{\Gamma} \ln \zeta(s) d s=\operatorname{Re}\left(\sum_{k=1}^{4} I_{k}+\sum_{\substack{\beta>1 / 2 \\ 0<\gamma<T}} I_{\varrho}\right)=0,
$$

где $I_{k}, k=1,2,3,4,-$ интегралы по сторнам прямоугольника, а $I_{\varrho}-$ интеграл вдоль разреза, проведенного к нулю $\varrho=\beta+i \gamma$. Поскольку при $\sigma \geqslant A$ и любом $t \geqslant 0$

$$
|\ln \zeta(\sigma+i t)|=\left|\sum_{n=2}^{+\infty} \frac{\Lambda(n)}{\ln n} n^{-\sigma-i t}\right| \leqslant \sum_{n=2}^{+\infty} n^{-\sigma}<2^{1-\sigma}
$$

и

$$
\left|\int_{0}^{t} \ln \zeta(\sigma+i t) d \sigma\right|=\left|\sum_{n=2}^{+\infty} \frac{\Lambda(n)}{\ln ^{2} n}\left(n^{-i t}-1\right) n^{-\sigma}\right|<3 \cdot 2^{-\sigma}
$$

то

$$
\begin{gathered}
\operatorname{Re} I_{1}=\operatorname{Re}\left(\text { v.p. } \int_{1 / 2}^{A} \ln \zeta(\sigma) d \sigma\right)=\operatorname{Re}\left(\text { v.p. } \int_{1 / 2}^{+\infty} \ln \zeta(\sigma) d \sigma+\theta \int_{A}^{+\infty} 2^{1-\sigma} d \sigma\right) \\
=\text { v.p. } \int_{1 / 2}^{+\infty} \ln |\zeta(\sigma)| d \sigma+3 \theta \cdot 2^{-A} \\
\qquad \operatorname{Re} I_{2}|\leqslant| I_{2}|=| \int_{0}^{T} \ln \zeta(A+i t) d t \mid<3 \cdot 2^{-A}
\end{gathered}
$$

и

$$
\operatorname{Re} I_{3}=-\operatorname{Re}\left(\int_{1 / 2}^{A} \ln \zeta(\sigma+i T) d \sigma\right)=- \text { v.p. } \int_{1 / 2}^{+\infty} \ln |\zeta(\sigma+i T)| d \sigma+3 \theta \cdot 2^{-A}
$$


Кроме того,

$$
\begin{aligned}
\operatorname{Re} I_{4} & =-\operatorname{Re}\left(i \int_{0}^{T} \ln \zeta(1 / 2+i t) d t\right) \\
& =-\operatorname{Re} \int_{0}^{T}(-\pi S(t)+i \ln |\zeta(1 / 2+i t)|) d t=\pi S_{1}(T) .
\end{aligned}
$$

Наконец, все интегралы $I_{\varrho}$ являются чисто мнимыми. Действительно,

$$
\begin{aligned}
\operatorname{Re} I_{\varrho} & =\operatorname{Re}\left(\int_{1 / 2}^{\beta} \ln \zeta(\sigma+i(\gamma+0)) d \sigma-\int_{1 / 2}^{\beta} \ln \zeta(\sigma+i(\gamma-0)) d \sigma\right) \\
& =\int_{1 / 2}^{\beta}(\ln |\zeta(\sigma+i(\gamma+0))|-\ln |\zeta(\sigma+i(\gamma-0))|) d \sigma \\
& =\int_{1 / 2}^{\beta}(\ln |\zeta(\sigma+i \gamma)|-\ln |\zeta(\sigma+i \gamma)|) d \sigma=0 .
\end{aligned}
$$

Таким образом,

$$
\pi S_{1}(T)+\text { v.p. } \int_{1 / 2}^{+\infty} \ln |\zeta(\sigma)| d \sigma-\text { v.p. } \int_{1 / 2}^{+\infty} \ln |\zeta(\sigma+i T)| d \sigma+9 \theta \cdot 2^{-A}=0 .
$$

Деля обе части полученного равенства на $\pi$ и устремляя $A$ к бесконечности, мы приходим к утверждению леммы.

Teopema 2. Пусть $e^{16}<x \leqslant t^{2}$. Тогдa

$$
\begin{aligned}
S_{1}(t)+\frac{1}{\pi} \text { v.p. } \int_{1 / 2}^{+\infty} \ln |\zeta(\sigma)| d \sigma=- & \frac{1}{\pi} \sum_{n \leqslant x^{3}} \frac{\Lambda_{x}(n)}{n^{\sigma_{x, t}}} \frac{\cos (t \ln n)}{(\ln n)^{2}}\left(1+\left(\sigma_{x, t}-\frac{1}{2}\right) \ln n\right) \\
& +5 \theta\left(\sigma_{x, t}-\frac{1}{2}\right)^{2}(|r(x, t)|+\ln t) .
\end{aligned}
$$

ДокАЗАТЕльСтво. Преобразуем левую часть равенства, пользуясь формулой леммы 8:

$$
\begin{aligned}
S_{1}(t)+\frac{1}{\pi} \text { v.p. } \int_{1 / 2}^{+\infty} \ln |\zeta(\sigma)| d \sigma & =\operatorname{Re}\left(\frac{1}{\pi} \text { v.p. } \int_{1 / 2}^{+\infty} \ln \zeta(\sigma+i t) d \sigma\right) \\
& =\operatorname{Re}\left(\frac{1}{\pi} \text { v.p. } \int_{1 / 2}^{+\infty} \ln \zeta(\sigma+i t) d\left(\sigma-\frac{1}{2}\right)\right) \\
& =-\operatorname{Re}\left(\frac{1}{\pi} \text { v.p. } \int_{1 / 2}^{+\infty}\left(\sigma-\frac{1}{2}\right) \frac{\zeta^{\prime}(\sigma+i t)}{\zeta(\sigma+i t)} d \sigma\right) \\
& =-\left(I_{1}+I_{2}+I_{3}\right)
\end{aligned}
$$


где обозначено:

$$
\begin{aligned}
& I_{1}=\frac{1}{\pi} \operatorname{Rev} \cdot \mathrm{p} \cdot \int_{\sigma_{x, t}}^{+\infty}\left(\sigma-\frac{1}{2}\right) \frac{\zeta^{\prime}(\sigma+i t)}{\zeta(\sigma+i t)} d \sigma \\
& I_{2}=\frac{1}{2 \pi}\left(\sigma-\frac{1}{2}\right)^{2} \operatorname{Re} \frac{\zeta^{\prime}\left(\sigma_{x, t}+i t\right)}{\zeta\left(\sigma_{x, t}+i t\right)}, \\
& I_{3}=\frac{1}{\pi} \mathrm{v} \cdot \mathrm{p} \cdot \int_{1 / 2}^{\sigma_{x, t}}\left(\sigma-\frac{1}{2}\right) \operatorname{Re}\left(\frac{\zeta^{\prime}(\sigma+i t)}{\zeta(\sigma+i t)}-\frac{\zeta^{\prime}\left(\sigma_{x, t}+i t\right)}{\zeta\left(\sigma_{x, t}+i t\right)}\right) d \sigma .
\end{aligned}
$$

Чтобы вычислить первый интеграл, воспользуемся выражением для логарифмической производной дзета-функции, которое дается леммой 7:

$$
\begin{aligned}
I_{1}= & -\frac{1}{\pi} \operatorname{Re}\left(\sum_{n \leqslant x^{3}} \frac{\Lambda_{x}(n)}{n^{i t}} \int_{\sigma_{x, t}}^{+\infty}\left(\sigma-\frac{1}{2}\right) n^{-\sigma} d \sigma\right) \\
& +\frac{13 \theta}{3 \pi}(|r(x, t)|+0.5 \ln t+4) \int_{\sigma_{x, t}}^{+\infty}\left(\sigma-\frac{1}{2}\right) x^{-\frac{1}{2}(\sigma-1 / 2)} d \sigma .
\end{aligned}
$$

Так как $\sigma_{x, t}-1 / 2 \geqslant 2(\ln x)^{-1}$ и при $u \geqslant 0(1+u) e^{-u} \leqslant 1$, то

$$
\begin{aligned}
& \int_{\sigma_{x, t}}^{+\infty}\left(\sigma-\frac{1}{2}\right) x^{-\frac{1}{2}(\sigma-1 / 2)} d \sigma \\
& \quad=\frac{4}{(\ln x)^{2}}\left(1+\frac{1}{2}\left(\sigma_{x, t}-\frac{1}{2}\right) \ln x\right) \exp \left(-\frac{1}{2}\left(\sigma_{x, t}-\frac{1}{2}\right) \ln x\right) \\
& \quad \leqslant\left(\sigma_{x, t}-\frac{1}{2}\right)^{2}
\end{aligned}
$$

и

$$
\begin{aligned}
I_{1}= & -\frac{1}{\pi} \sum_{n \leqslant x^{3}} \frac{\Lambda_{x}(n)}{n^{\sigma_{x, t}}} \frac{\cos (t \ln n)}{(\ln n)^{2}}\left(1+\left(\sigma_{x, t}-\frac{1}{2}\right) \ln n\right) \\
& +\frac{13 \theta}{3 \pi}\left(\sigma_{x, t}-\frac{1}{2}\right)^{2}(|r(x, t)|+0.5 \ln |t|+4) .
\end{aligned}
$$

Оценивая $I_{2}$, воспользуемся следствием леммы 7:

$$
\left|I_{2}\right| \leqslant \frac{1}{2 \pi}\left(\sigma_{x, t}-\frac{1}{2}\right)^{2}\left|\frac{\zeta^{\prime}\left(\sigma_{x, t}+i t\right)}{\zeta\left(\sigma_{x, t}+i t\right)}\right| \leqslant \frac{1}{2 \pi}\left(\sigma_{x, t}-\frac{1}{2}\right)^{2}(1.3|r(x, t)|+0.4 \ln |t|+2.5) .
$$


Рассмотрим теперь интеграл $I_{3}$. Преобразуя подьнтегральное выражение с помошью формулы леммы 2 , получим:

$$
\begin{aligned}
I_{3}= & \frac{1}{\pi} \int_{1 / 2}^{\sigma_{x, t}}\left(\sigma-\frac{1}{2}\right) \sum_{\varrho}\left(\frac{\sigma-\beta}{(\sigma-\beta)^{2}+(t-\gamma)^{2}}-\frac{\sigma_{x, t}-\beta}{\left(\sigma_{x, t}-\beta\right)^{2}+(t-\gamma)^{2}}\right) d \sigma \\
& +\frac{\theta}{2 \pi}\left(\sigma_{x, t}-\frac{1}{2}\right)^{2}(\ln |t|+6) \\
= & \frac{1}{\pi} \sum_{\varrho} \frac{1}{\left(\sigma_{x, t}-\beta\right)^{2}+(t-\gamma)^{2}} \\
& \times \int_{1 / 2}^{\sigma_{x, t}} \frac{(\sigma-1 / 2)\left(\sigma_{x, t}-\sigma\right)\left(\left(\sigma_{x, t}-\beta\right)(\sigma-\beta)-(t-\gamma)^{2}\right)}{(\sigma-\beta)^{2}+(t-\gamma)^{2}} d \sigma \\
& +\frac{\theta}{2 \pi}\left(\sigma_{x, t}-\frac{1}{2}\right)^{2}(\ln |t|+6) .
\end{aligned}
$$

Обозначим интеграл по $\sigma$ символом $\varkappa(\varrho)$ и покажем, что $\varkappa(\varrho) \leqslant 2\left(\sigma_{x, t}-1 / 2\right)^{2}$. Для этого рассмотрим три случая. Пусть сначала $|\beta-1 / 2|>\frac{1}{2}\left(\sigma_{x, t}-1 / 2\right)$ или, что то же, $\sigma_{x, t}<1 / 2+2|\beta-1 / 2|$. В этом случае

$$
|t-\gamma|>\frac{x^{3|\beta-1 / 2|}}{\ln x}>3\left|\beta-\frac{1}{2}\right|
$$

и для всех $\sigma$ из промежутка $1 / 2 \leqslant \sigma \leqslant \sigma_{x, t}$

$$
|\sigma-\beta| \leqslant\left|\sigma-\frac{1}{2}\right|+\left|\beta-\frac{1}{2}\right| \leqslant\left|\sigma_{x, t}-\frac{1}{2}\right|+\left|\beta-\frac{1}{2}\right|<2\left|\beta-\frac{1}{2}\right|+\left|\beta-\frac{1}{2}\right|<|t-\gamma| .
$$

Следовательно,

$$
\begin{aligned}
|\varkappa(\varrho)| & \leqslant \int_{1 / 2}^{\sigma_{x, t}} \frac{(\sigma-1 / 2)\left(\sigma_{x, t}-\sigma\right)\left((t-\gamma)^{2}+(t-\gamma)^{2}\right)}{(\sigma-\beta)^{2}+(t-\gamma)^{2}} d \sigma \\
& <2\left(\sigma_{x, t}-\frac{1}{2}\right)^{2} \int_{1 / 2}^{\sigma_{x, t}} \frac{(t-\gamma)^{2}}{(\sigma-\beta)^{2}+(t-\gamma)^{2}} d \sigma \\
& \leqslant 2\left(\sigma_{x, t}-\frac{1}{2}\right)^{2} \int_{1 / 2}^{\sigma_{x, t}} \frac{(\sigma-\beta)^{2}+(t-\gamma)^{2}}{(\sigma-\beta)^{2}+(t-\gamma)^{2}} d \sigma=2\left(\sigma_{x, t}-\frac{1}{2}\right)^{3}
\end{aligned}
$$

Пусть, далее, $\frac{1}{2}\left(1 / 2-\sigma_{x, t}\right) \leqslant \beta-1 / 2 \leqslant 0$. Тогда $\beta \leqslant 1 / 2, \sigma-\beta \geqslant \sigma-1 / 2 \geqslant 0$,

и

$$
0 \leqslant \sigma_{x, t}-\beta \leqslant\left(\sigma_{x, t}-\frac{1}{2}\right)+\left|\beta-\frac{1}{2}\right| \leqslant \frac{3}{2}\left(\sigma_{x, t}-\frac{1}{2}\right)
$$

$$
\begin{aligned}
|\varkappa(\varrho)| \leqslant & \int_{1 / 2}^{\sigma_{x, t}}\left(\left(\sigma-\frac{1}{2}\right)(t-\gamma)^{2}+\left(\sigma-\frac{1}{2}\right)(\sigma-\beta)\left(\sigma_{x, t}-\beta\right)\right) \frac{\left(\sigma_{x, t}-\beta\right) d \sigma}{(\sigma-\beta)^{2}+(t-\gamma)^{2}} \\
\leqslant & \left(\sigma_{x, t}-\frac{1}{2}\right)\left(\int_{1 / 2}^{\sigma_{x, t}}\left(\sigma-\frac{1}{2}\right) \frac{(t-\gamma)^{2} d \sigma}{(\sigma-\beta)^{2}+(t-\gamma)^{2}}\right. \\
& \left.+\frac{3}{2}\left(\sigma_{x, t}-\frac{1}{2}\right) \int_{1 / 2}^{\sigma_{x, t}} \frac{(\sigma-\beta)^{2} d \sigma}{(\sigma-\beta)^{2}+(t-\gamma)^{2}}\right) \\
\leqslant & \frac{1}{2}\left(\sigma_{x, t}-\frac{1}{2}\right)^{3}+\frac{3}{2}\left(\sigma_{x, t}-\frac{1}{2}\right)^{3}=2\left(\sigma_{x, t}-\frac{1}{2}\right)^{3} .
\end{aligned}
$$


Рассмотрим, наконец, случай, когда $1 / 2<\beta \leqslant \frac{1}{2}\left(\sigma_{x, t}+1 / 2\right)$. Представляя $\varkappa(\varrho)$ в виде разности

$$
\begin{gathered}
\int_{1 / 2}^{\sigma_{x, t}} \frac{\left(\sigma_{x, t}-\beta\right)\left(\sigma+\beta-\sigma_{x, t}-1 / 2\right)(\sigma-\beta)^{2}+(\sigma-1 / 2)\left(\sigma_{x, t}-\sigma\right)(t-\gamma)^{2}}{(\sigma-\beta)^{2}+(t-\gamma)^{2}} d \sigma \\
-\int_{1 / 2}^{\sigma_{x, t}} \frac{\left(\sigma_{x, t}-\beta\right)^{2}(\beta-1 / 2)(\sigma-\beta)}{(\sigma-\beta)^{2}+(t-\gamma)^{2}} d \sigma
\end{gathered}
$$

и замечая, что $\left|\sigma_{x, t}-\beta\right| \leqslant \sigma_{x, t}-1 / 2,\left|\sigma+\beta-\sigma_{x, t}-1 / 2\right| \leqslant \sigma_{x, t}-1 / 2$, получим:

$$
\begin{aligned}
|\varkappa(\varrho)| \leqslant & \int_{1 / 2}^{\sigma_{x, t}} \frac{\left(\sigma_{x, t}-1 / 2\right)^{2}(\sigma-\beta)^{2}+\left(\sigma_{x, t}-1 / 2\right)^{2}(t-\gamma)^{2}}{(\sigma-\beta)^{2}+(t-\gamma)^{2}} d \sigma \\
& +\left(\sigma_{x, t}-\beta\right)^{2}\left(\beta-\frac{1}{2}\right) \int_{1 / 2}^{\sigma_{x, t}} \frac{(\sigma-\beta) d \sigma}{(\sigma-\beta)^{2}+(t-\gamma)^{2}} \\
\leqslant & \left(\sigma_{x, t}-\frac{1}{2}\right)^{3}+\left(\sigma_{x, t}-\beta\right)^{2}\left(\beta-\frac{1}{2}\right) \cdot \frac{1}{2} \ln \frac{\left(\sigma_{x, t}-\beta\right)^{2}+(t-\gamma)^{2}}{(\beta-1 / 2)^{2}+(t-\gamma)^{2}} .
\end{aligned}
$$

Так как $\left(\sigma_{x, t}-\beta\right)^{2} \geqslant(\beta-1 / 2)^{2}$, то дробь под знаком логарифма не превосходит

$$
\left(\frac{\sigma_{x, t}-\beta}{\beta-1 / 2}\right)^{2}
$$

и поэтому

$$
\begin{aligned}
|\varkappa(\varrho)| & \leqslant\left(\sigma_{x, t}-\frac{1}{2}\right)^{3}+\left(\sigma_{x, t}-\beta\right)^{2}\left(\beta-\frac{1}{2}\right) \ln \frac{\sigma_{x, t}-\beta}{\beta-1 / 2} \\
& =\left(\sigma_{x, t}-\frac{1}{2}\right)^{3}\left(1+\frac{\beta-1 / 2}{\sigma_{x, t}-1 / 2} \ln \frac{\sigma_{x, t}-1 / 2}{\beta-1 / 2}\right)<2\left(\sigma_{x, t}-\frac{1}{2}\right)^{3} .
\end{aligned}
$$

Итак, в каждом из трех случаев $\varkappa(\varrho)$ по модулю не превосходит $2\left(\sigma_{x, t}-1 / 2\right)^{3}$. Следовательно,

$$
\begin{aligned}
\left|I_{3}\right| & \leqslant \frac{2}{\pi}\left(\sigma_{x, t}-\frac{1}{2}\right)^{3} \cdot \frac{13}{3}\left(\sigma_{x, t}-\frac{1}{2}\right)^{-1}(|r(x, t)|+0.5 \ln |t|+4) \\
& =\frac{26}{3 \pi}\left(\sigma_{x, t}-\frac{1}{2}\right)^{2}(|r(x, t)|+0.5 \ln |t|+4) .
\end{aligned}
$$

Складьвая полученные оценки, мы приходим к неравенству

$$
\begin{gathered}
\left|S_{1}(t)+\frac{1}{\pi} \mathrm{v} \cdot \mathrm{p} \cdot \int_{1 / 2}^{+\infty} \ln \right| \zeta(\sigma)\left|d \sigma+\frac{1}{\pi} \sum_{n \leqslant x^{3}} \frac{\Lambda_{x}(n)}{n^{\sigma_{x, t}}} \frac{\cos (t \ln n)}{(\ln n)^{2}}\left(1+\left(\sigma_{x, t}-\frac{1}{2}\right) \ln n\right)\right| \\
\leqslant\left(\sigma_{x, t}-\frac{1}{2}\right)^{2}\left(c_{1}|r(x, t)|+c_{2} \ln |t|+c_{3}\right)
\end{gathered}
$$


где

$$
c_{1}=\frac{13}{3 \pi}+\frac{1.3}{\pi}+\frac{26}{3 \pi}, \quad c_{2}=\frac{13}{6 \pi}+\frac{0.4}{\pi}+\frac{13}{3 \pi}, \quad c_{3}=\frac{52}{3 \pi}+\frac{2.5}{\pi}+\frac{104}{3 \pi} .
$$

Пользуясь тем, что $c_{1}<5, c_{2}<2.5, c_{3}<20$, и тем, что при $|t| \geqslant e^{8}$

$$
\frac{1}{2} \ln |t|+4<\ln |t|,
$$

мы приходим к неравенству, содержашемуся в утверждении теоремы. Теорема доказана.

\section{§. Несобственный интеграл, содержащий функцию $S(t)$}

ОПРЕДЕЛЕнИЕ 3 . Пусть $f(z)$ - функция, вешественная на вешественной прямой, аналитическая в полосе $|\operatorname{Im} z| \leqslant 1$ и удовлетворяющая там условию $|f(z)| \leqslant$ $c(|z|+1)^{-(1+\alpha)}$, где $c$ и $\alpha$ - положительные абсолютные постоянные. Для вещественного $x$ символом $\widehat{f}(x)$ обозначим интеграл

$$
\int_{-\infty}^{+\infty} f(u) e^{-i x u} d u
$$

Лемма 9. Для любого $n \geqslant 1$ справедливы соотношения:
1) $n^{-1} \int_{-\infty}^{+\infty} f(x-i) n^{-i x} d x=\widehat{f}(\ln n)$
2) $|\widehat{f}(\ln n)| \leqslant 2 c \alpha^{-1} n^{-1}$.

ДокАЗАТЕльство. Пусть $\Gamma$ - граница прямоугольника с вершинами в точках $\pm R$, $\pm R-i, R>1$, обходимая против часовой стрелки. По теореме Коши о вычетах,

$$
I=\int_{\Gamma} f(z) n^{-i z} d z=0 .
$$

Представим $I$ в виде суммы $\sum_{k=1}^{4} I_{k}$, в которой $I_{1}$ и $I_{2}-$ интегралы по верхней и нижней сторонам прямоугольника, а $I_{3}, I_{4}$ - интегралы по его боковым сторонам. Тогда

$$
\begin{gathered}
I_{1}=-\int_{-R}^{R} f(x) e^{-i x \ln n} d x, \\
I_{2}=\int_{-R}^{R} f(x-i) e^{-i(x-i) \ln n} d x=n^{-1} \int_{-R}^{R} f(x-i) e^{-i x \ln n} d x, \\
\left|I_{3}\right|,\left|I_{4}\right| \leqslant\left|\int_{0}^{1} f( \pm R-i u) e^{-i( \pm R-i u) \ln n} d u\right| \\
\leqslant \int_{0}^{1}|f( \pm R-i u)| d u \leqslant c(R+1)^{-(1+\alpha)} .
\end{gathered}
$$

Устремляя теперь в равенстве $I_{2}=-\left(I_{1}+I_{3}+I_{4}\right) R$ к бесконечности, получим:

$$
\begin{gathered}
n^{-1} \int_{-\infty}^{+\infty} f(x-i) e^{-i x \ln n} d x=\int_{-\infty}^{+\infty} f(x) e^{-i x \ln n} d x=\widehat{f}(\ln n), \\
|\widehat{f}(\ln n)| \leqslant n^{-1} \int_{-\infty}^{+\infty}|f(x-i)| d x \leqslant c n^{-1} \int_{-\infty}^{+\infty} \frac{d x}{(1+|x|)^{1+\alpha}}=2 c \alpha^{-1} n^{-1} .
\end{gathered}
$$

Лемма доказана. 
ТЕОРема 3. Пусть $f(z)$ - функиия, вещественная на вещественной прямой, аналитическая в полосе $|\operatorname{Im} z| \leqslant 1$ и удовлетворяющая там неравенству $|f(z)| \leqslant$ $c(|z|+1)^{-(1+\alpha)}, c>0, \alpha>0$. Тогда при любом $t$ справедлива формула:

$$
\begin{gathered}
\int_{-\infty}^{+\infty} f(u) \ln \zeta\left(\frac{1}{2}+i(t+u)\right) d u \\
=\sum_{n=2}^{+\infty} \frac{\Lambda(n)}{\ln n} \frac{n^{-i t}}{\sqrt{n}} \widehat{f}(\ln n)+2 \pi\left(\sum_{\beta>1 / 2} \int_{0}^{\beta-1 / 2} f(\gamma-t-i \xi) d \xi-\int_{0}^{1 / 2} f(-t-i \xi) d \xi\right)
\end{gathered}
$$

где в последней сумме суммирование ведется по всем комплексным нулям Ц(s), лежащим правее критической прямой.

ДокАЗАТЕЛЬСТВо. Положим $\psi(z)=f(i(1 / 2-z)-t)$ и возьмем $X \geqslant 2(|t|+10)$ так, чтобы ординаты всех нулей $\zeta(s)$ отстояли от $X$ на расстояние, не меньшее $c(\ln X)^{-1}$, где $c$ - некоторая положительная абсолютная постоянная. Такой выбор возможен в силу следствия леммы 3.

Пусть $\Gamma$ - граница прямоугольника с вершинами $1 / 2 \pm i X, 3 / 2 \pm i X$, в котором к каждому из нулей $\varrho=\beta+i \gamma$, а также к точке $z=1$ от критической прямой проведен горизонтальный разрез. Тогда $\ln \zeta(z)$ и $\psi(z)$ аналитичны внутри $Г$. Согласно теореме Коши о вычетах, имеет место следуюшее равенство:

$$
\begin{aligned}
0 & =\int_{\Gamma} \psi(z) \ln \zeta(z) d z \\
& =\left(\int_{3 / 2-i X}^{3 / 2+i X}-\int_{1 / 2+i X}^{3 / 2+i X}-\int_{1 / 2-i X}^{1 / 2+i X}+\int_{1 / 2-i X}^{3 / 2-i X}\right) \psi(z) \ln \zeta(z) d z \\
& =I_{1}-I_{2}-I_{3}+I_{4}
\end{aligned}
$$

Полагая в выражении для $I_{1} z=3 / 2+i x$, находим:

$$
\begin{aligned}
I_{1} & =i \int_{-X}^{X} f(x-t-i) \ln \zeta\left(\frac{3}{2}+i x\right) d x \\
& =i\left(\int_{-\infty}^{+\infty}-\int_{-\infty}^{-X}-\int_{X}^{+\infty}\right) f(x-t-i) \ln \zeta\left(\frac{3}{2}+i x\right) d x
\end{aligned}
$$

Поскольку при $|x| \geqslant X|x-t| \geqslant 0.5|x|$ и $\ln \zeta(3 / 2+i x)=O(1)$, то два последних интеграла по порядку не превосходят величины

$$
\int_{X}^{+\infty} \frac{d x}{(1+0.5 x)^{1+\alpha}}=O\left(X^{-\alpha}\right) .
$$

Для вьчисления несобственного интеграла воспользуемся леммой 9:

$$
\begin{aligned}
I_{1} & =i \sum_{n=2}^{+\infty} \frac{\Lambda(n)}{\ln n} \frac{1}{n \sqrt{n}} \int_{-\infty}^{+\infty} f(x-t-i) n^{-i x} d x+O\left(X^{-\alpha}\right) \\
& =i \sum_{n=2}^{+\infty} \frac{\Lambda(n)}{\ln n} \frac{n^{-i t}}{\sqrt{n}} \widehat{f}(\ln n)+O\left(X^{-\alpha}\right) .
\end{aligned}
$$


Чтобы оценить интегралы $I_{2}$ и $I_{3}$, заметим, что при $1 / 2 \leqslant \sigma_{1} \leqslant 3 / 2$

$$
\begin{aligned}
\left|\ln \zeta\left(\sigma_{1}+i X\right)\right| & =\left|\sum_{|\gamma-X| \leqslant 1} \ln \left(\sigma_{1}-\beta+i(X-\gamma)\right)\right|+O(\ln X) \\
& =O((\ln X) \ln \ln X)=O\left(\ln ^{2} X\right)
\end{aligned}
$$

(см. [20; с. 116]). Поэтому

$$
\begin{aligned}
\left|I_{2}\right| & =\left|\int_{1 / 2}^{3 / 2} f\left(X-t+i\left(\sigma-\sigma_{1}\right)\right) \ln \zeta\left(\sigma_{1}+i X\right) d \sigma_{1}\right| \\
& =O\left(\ln ^{2} X \int_{1 / 2}^{3 / 2}\left|f\left(X-t+i\left(\sigma-\sigma_{1}\right)\right)\right| d \sigma_{1}\right)=O\left(X^{-(1+\alpha)} \ln ^{2} X\right) .
\end{aligned}
$$

Аналогично доказьвается, что $I_{4}=O\left(X^{-(1+\alpha)} \ln ^{2} X\right)$. Вычислим теперь интеграл $I_{3}$. Пусть $\gamma$ является ординатой для $k$ различных нулей $\varrho_{1}, \ldots, \varrho_{k}$ с кратностями $m_{1}, \ldots, m_{k}$, лежаших правее критической прямой, и пусть $\beta_{1}, \ldots, \beta_{k}$ - их вещественные части, упорядоченные в порядке возрастания. Обозначим буквой $L$ разрез, идуший от точки $1 / 2+i \gamma$ к точке $\beta_{k}+i \gamma$, а символом $I(L)-$ интеграл по берегам этого разреза. Тогда

$$
\begin{aligned}
I(L) & =\int_{L} \psi(z) \ln \zeta(z) d z=\left(\int_{1 / 2+i(\gamma+0)}^{\beta_{k}+i(\gamma+0)}+\int_{\beta_{k}+i(\gamma-0)}^{1 / 2+i(\gamma-0)}\right) \psi(z) \ln \zeta(z) d z \\
& =\int_{1 / 2}^{\beta_{k}}(\psi(x+i(\gamma+0)) \ln \zeta(x+i(\gamma+0))-\psi(x+i(\gamma-0)) \ln \zeta(x+i(\gamma-0))) d x \\
& =\int_{1 / 2}^{\beta_{k}} \psi(x+i \gamma)(\ln \zeta(x+i(\gamma+0))-\ln \zeta(x+i(\gamma-0))) d x \\
& =\int_{1 / 2}^{\beta_{k}} \psi(x+i \gamma)(\ln |\zeta(x+i \gamma)|+i \arg \zeta(x+i(\gamma+0)) \\
& =i \int_{1 / 2}^{\beta_{k}} \psi(x+i \gamma) h(x) d x
\end{aligned}
$$

где символом $h(x)$ обозначена разность

$$
\arg \zeta(x+i(\gamma+0))-\arg \zeta(x+i(\gamma-0))
$$

Для того чтобы вычислить последний интеграл, представим $\zeta(z)$ в виде произведения

$$
\left(z-\varrho_{1}\right)^{m_{1}} \cdots\left(z-\varrho_{k}\right)^{m_{k}} g(z),
$$


где функция $g(z)$ не обращается в нуль в некоторой окрестности отрезка с конщами в точках $1 / 2+i \gamma, 3 / 2+i \gamma$. Тогда

$$
\begin{aligned}
h(x)= & \sum_{\nu=1}^{k} m_{\nu}\left(\arg \left(x+i(\gamma+0)-\beta_{\nu}-i \gamma\right)-\arg \left(x+i(\gamma-0)-\beta_{\nu}-i \gamma\right)\right) \\
& \quad+\arg g(x+i(\gamma+0))-\arg g(x+i(\gamma-0)) \\
= & \sum_{\nu=1}^{k} m_{\nu}\left(\arg \left(x-\beta_{\nu}+i \cdot 0\right)-\arg \left(x-\beta_{\nu}-i \cdot 0\right)\right) .
\end{aligned}
$$

Поскольку разность $\arg \left(x-\beta_{\nu}+i \cdot 0\right)-\arg \left(x-\beta_{\nu}-i \cdot 0\right)$ равна 0 , если $x>\beta_{\nu}$, равна $\pi$, если $x=\beta_{\nu}$, и равна $2 \pi$, если $x<\beta_{\nu}$, то при $\beta_{\nu}<x<\beta_{\nu+1}$

$$
h(x)=2 \pi\left(m_{\nu+1}+m_{\nu+2}+\cdots+m_{k}\right) .
$$

Следовательно,

$$
\begin{aligned}
I(L)= & 2 \pi i\left(\left(m_{1}+\cdots+m_{k}\right) \int_{1 / 2}^{\beta_{1}} \psi(x+i \gamma) d x\right. \\
& \left.+\left(m_{2}+\cdots+m_{k}\right) \int_{\beta_{1}}^{\beta_{2}} \psi(x+i \gamma) d x+\cdots+m_{k} \int_{\beta_{k-1}}^{\beta_{k}} \psi(x+i \gamma) d x\right) \\
= & 2 \pi i \sum_{\substack{\operatorname{Im} \varrho=\gamma \\
\beta=\operatorname{Re} \varrho>1 / 2}} \int_{1 / 2}^{\beta} \psi(x+i \gamma) d x=2 \pi i \sum_{\substack{\operatorname{Im} \varrho=\gamma \\
\beta=\operatorname{Re} \varrho>1 / 2}} \int_{0}^{\beta-1 / 2} f(\gamma-t-i x) d x .
\end{aligned}
$$

Если $L$ - разрез, идущий к точке $z=1$, то

$$
I(L)=-2 \pi i \int_{0}^{1 / 2} f(-t-i x) d x .
$$

Таким образом, интеграл $I_{3}$ принимает вид:

$$
\begin{aligned}
I_{3}= & \int_{1 / 2-i X}^{1 / 2+i X} \psi(z) \ln \zeta(z) d z \\
& -2 \pi i\left(\sum_{\substack{|\gamma|<X \\
\beta>1 / 2}} \int_{0}^{\beta-1 / 2} f(\gamma-t-i x) d x-\int_{0}^{1 / 2} f(-t-i x) d x\right) .
\end{aligned}
$$

В равенстве

$$
I_{1}=I_{3}+O\left(X^{-\alpha}\right)+O\left(X^{-(1+\alpha)} \ln ^{2} X\right)
$$

устремим $X$ к бесконечности. Получим:

$$
\begin{aligned}
\int_{1 / 2-i \infty}^{1 / 2+\infty} \psi(z) \ln \zeta(z) d z=i \int_{-\infty}^{+\infty} f(u-t) \ln \zeta(1 / 2+i u) d u \\
=i \sum_{n=2}^{+\infty} \frac{\Lambda(n)}{\ln n} \frac{n^{-i t}}{\sqrt{n}} \widehat{f}(\ln n) \\
\quad+2 \pi i\left(\sum_{\beta>1 / 2} \int_{0}^{\beta-1 / 2} f(\gamma-t-i x) d x-\int_{0}^{1 / 2} f(-t-i x) d x\right) .
\end{aligned}
$$


Отсюда следует равенство:

$$
\begin{aligned}
& \int_{-\infty}^{+\infty} f(u) \ln \zeta(1 / 2+i(t+u)) d u=\sum_{n=2}^{+\infty} \frac{\Lambda(n)}{\ln n} \frac{n^{-i t}}{\sqrt{n}} \widehat{f}(\ln n) \\
& \quad+2 \pi\left(\sum_{\beta>1 / 2} \int_{0}^{\beta-1 / 2} f(\gamma-t-i x) d x-\int_{0}^{1 / 2} f(-t-i x) d x\right) .
\end{aligned}
$$

Теорема доказана.

СлЕДСТВИЕ. При тех же предположсениях относительно функиии $f$ справедлива формула:

$$
\begin{aligned}
& \int_{-\infty}^{+\infty} f(u) S(t+u) d u=\frac{1}{\pi} \sum_{n=2}^{+\infty} \frac{\Lambda(n)}{\sqrt{n} \ln n} \operatorname{Im}\left(n^{-i t} \widehat{f}(\ln n)\right) \\
& \quad+2\left(\sum_{\beta>1 / 2} \int_{0}^{\beta-1 / 2} \operatorname{Im} f(\gamma-t-i x) d x-\int_{0}^{1 / 2} \operatorname{Im} f(-t-i x) d x\right) .
\end{aligned}
$$

\section{Замечания}

Теоремы 1 и 2 принадлежат А. Сельбергу. Им же введены функции $\Lambda_{x}(n)$ и $\sigma_{x, t}$. Приведенный здесь способ доказательства этих теорем (а также лемм 5,6 и 7) содержится в работе [27]. Аналог тождества леммы 5, связьваюшего нули дзета-функции Римана с функцией Мангольдта, можно найти в [20; с. 359-360] и [28; с. 58-59].

Формула леммы 8 впервые получена Дж. Литтлвудом [29] в 1924 г. (см. также леммy $5 \Pi)$.

Частньй случай формулы теоремы 3 использован А. Сельбергом в [27] при доказательстве омега-теорем для функции $S(t)$. Доказательство обшей формулы содержится в работе К. Тсанга [30].

Другой вариант приближенной формулы для $S(t)$ предложен в 1987 г. Д. Голдстоном [31] и имеет вид:

$$
\begin{aligned}
S(t)= & -\frac{1}{\pi} \sum_{n \leqslant x} \frac{\Lambda(n)}{\sqrt{n}} \frac{\sin (t \ln n)}{\ln n} f\left(\frac{\ln n}{\ln x}\right) \\
& -\frac{\sqrt{x}}{\pi} \operatorname{Im} \int_{0}^{+\infty} \frac{x^{-i t}}{((1 / 2-i t) \ln x)^{2}-u^{2}} \frac{u d u}{\operatorname{sh}(u)}+O\left(t^{-1} \ln ^{-2} x\right),
\end{aligned}
$$

где $t \geqslant 1, t \neq \gamma, x \geqslant 4$, a $f(u)=\frac{\pi u}{2} \operatorname{ctg}\left(\frac{\pi u}{2}\right)$. 
Глава III. Рост функий $|S(t)| \mathbf{и}\left|S_{1}(t)\right|$

Одним из основных направлений исследований функции $S(t)$ является изучение величин

$$
\inf _{0<t \leqslant T} S(t) \quad \text { и } \sup _{0<t \leqslant T} S(t)
$$

при $T \rightarrow+\infty$. Правильный порядок роста этих функций до сих пор не известен.

В первом параграфе настояшей главы даются верхние оценки величин $|S(t)|$ и $\left|S_{1}(t)\right|$, как безусловные, так и используюшие гипотезы Римана и Линделёфа. Второй параграф посвяшен доказательствам наиболее точных на сегодняшний день омега-теорем для функции $S(t)$.

\section{$\S$ 1. Верхние оценки $|S(t)|$ и $\left|S_{1}(t)\right|$.}

\section{Следствия гипотез Римана и Линделёфа}

ТеОРема 1. При $t \geqslant t_{0}>0$ справедливо неравенство $|S(t)|<8 \ln t$. Если верна гипотеза Римана, то

$$
|S(t)|<61 \frac{\ln t}{\ln \ln t}
$$

ДокАЗАТЕльство. Положим в приближенной формуле Сельберга (теорема 1 гл. II) $x=\sqrt{\ln t}$. Поскольку $\Lambda_{x}(n) \leqslant \ln n$, то

$$
\begin{aligned}
|S(t)| & \leqslant 15\left(\sum_{n \leqslant x^{3}} \frac{1}{\sqrt{n}} \frac{\Lambda_{x}(n)}{\ln n}+\sum_{n \leqslant x^{3}} \frac{\Lambda_{x}(n)}{\sqrt{n}}+\left(\sigma_{x, t}-0.5\right) \ln t\right) \\
& \leqslant 15\left(\sum_{n \leqslant x^{3}} \frac{\ln n}{\sqrt{n}}+\left(\sigma_{x, t}-0.5\right) \ln t\right) \\
& \leqslant 15\left(12 x^{1.5} \ln x+\left(\sigma_{x, t}-0.5\right) \ln t\right)<15\left(\sigma_{\sqrt{\ln t}, t}-0.5\right) \ln t+(\ln t)^{0.8} .
\end{aligned}
$$

Так как $\sigma_{x, t}-0.5 \leqslant 0.5$, то $|S(t)|<7.5 \ln t+(\ln t)^{0.8}<8 \ln t$. Если гипотеза Римана верна, то $\sigma_{x, t}-0.5=2 \ln ^{-1} x$, и

$$
|S(t)| \leqslant \frac{60 \ln t}{\ln \ln t}+(\ln t)^{0.8}<\frac{61 \ln t}{\ln \ln t} .
$$

Теорема доказана.

СлеДСТВИЕ. При $T>T_{0}>0$ имеет место следующая асимптотическая формула:

$$
N(T)=\frac{T}{2 \pi} \ln \frac{T}{2 \pi}-\frac{T}{2 \pi}+8.9 \theta \ln T, \quad|\theta|<1 .
$$

ТеОРема 2. При $t \geqslant t_{0}>0$ выполняется неравенство $\left|S_{1}(t)\right|<1.2 \ln t$. Если верна гипотеза Римана, то

$$
\left|S_{1}(t)\right|<40 \frac{\ln t}{(\ln \ln t)^{2}}
$$


ДокАЗАТЕЛЬСТво. Положив в приближенной формуле Сельберга для $S_{1}(t)$ (теорема 2 гл. II) $x=\sqrt{\ln t}$, получим:

$$
\begin{aligned}
\left|S_{1}(t)+c\right| \leqslant & \frac{1}{\pi} \sum_{2 \leqslant n \leqslant x^{3}} \frac{1}{\sqrt{n} \ln n} \\
& +\left(\frac{1}{2 \pi}+\frac{9}{8}\right) \sum_{2 \leqslant n \leqslant x^{3}} \frac{1}{\sqrt{n}}+\frac{9}{2}\left(\frac{1}{2}\right)^{2} 4+\frac{9}{4}(\sigma \sqrt{\ln t}, t-0.5)^{2} \ln t \\
& \leqslant \frac{9}{4}\left(\sigma_{\sqrt{\ln t}, t}-0.5\right)^{2} \ln t+3.5(\ln t)^{3 / 4}
\end{aligned}
$$

где $c=\pi^{-1} \int_{0.5}^{+\infty} \ln |\zeta(\sigma)| d \sigma$. Так как $\sigma_{x, t}-0.5 \leqslant 0.5$, то

$$
\left|S_{1}(t)\right| \leqslant \frac{9}{4} \cdot \frac{1}{4} \ln t+3.5(\ln t)^{3 / 4}+|c|<1.2 \ln t .
$$

Если верна гипотеза Римана, то $\sigma_{x, t}-0.5=2 \ln ^{-1} x$ и

$$
\left|S_{1}(t)\right| \leqslant \frac{9}{4} \frac{16 \ln t}{(\ln \ln t)^{2}}+3.5(\ln t)^{3 / 4}+|c|<40 \frac{\ln t}{(\ln \ln t)^{2}} .
$$

Теорема доказана.

Теорема 3. Если гипотеза Линделёфа верна, то $S(t)=o(\ln t)$ при $t \rightarrow+\infty$.

ДокАЗАТЕльСТво. Пусть $\varepsilon$ - сколь угодно малое фиксированное число, $\varepsilon>0$. Если верна гипотеза Линделёфа, то при всех $t \geqslant t_{0}(\varepsilon)>0$ и $\sigma>0.5$ справедливо неравенство (см. [20; с. 323], [28; с. 154]):

$$
|\zeta(\sigma+i t)| \leqslant t^{\varepsilon^{3}}
$$

Предположим сначала, что $t$ отлично от ординаты нуля $\zeta(s)$ и $t \geqslant t_{0}(\varepsilon)$. Величина $\pi S(t)$ равна сумме прирашений $\arg \zeta(s)$ вдоль отрезков $(2,2+i t)$ и $(2+i t, 0.5+i t)$. Прирашение вдоль вертикального отрезка не превосходит

$$
|\operatorname{Im}(\ln \zeta(2+i t)-\ln \zeta(2))|=\left|\sum_{n=2}^{+\infty} \frac{\Lambda(n)}{\ln n} \frac{\sin (t \ln n)}{n^{2}}\right| \leqslant \frac{\pi^{2}}{6}-1
$$

Пусть $\operatorname{Re} \zeta(\sigma+i t)$ меняет знак в точках $\sigma_{1}, \ldots, \sigma_{q}, 0.5 \leqslant \sigma_{1}<\cdots<\sigma_{q} \leqslant 2$. Эти точки разбивают горизонтальньй отрезок изменения $s$ на $\leqslant q+1$ интервалов, в каждом из которых $\operatorname{Re} \zeta(\sigma+i t)$ сохраняет знак. Прирашение $\arg \zeta(\sigma+i t)$ на каждом из этих интервалов не превосходит $\pi$. Следовательно,

$$
|S(t)| \leqslant q+1+\frac{1}{\pi}\left(\frac{\pi^{2}}{6}-1\right)<q+\frac{3}{2} .
$$


Оценим сверху величину $q$. Пусть $n(r)$ обозначает число нулей функции

$$
f(s)=\frac{1}{2}(\zeta(s+2+i t)+\zeta(s+2-i t))
$$

в круге $|s| \leqslant r$. Так как точки $\sigma_{1}, \ldots, \sigma_{q}$ являются вещественными нулями $f(s)$, то $q \leqslant n(1.5)$. Положим $R=1.5+\varepsilon^{2}$. Согласно формуле Иенсена (см. лемму $6 \Pi$ ),

$$
\begin{aligned}
\int_{0}^{R} \frac{n(r)}{r} d r= & \frac{1}{2 \pi} \int_{0}^{2 \pi} \ln \left|f\left(R e^{i \varphi}\right)\right| d \varphi-\ln |f(0)| \\
= & \frac{1}{2 \pi} \int_{0}^{2 \pi} \ln \left|\frac{1}{2}\left(\zeta\left(2+i t+R e^{i \varphi}\right)+\zeta\left(2-i t+R e^{i \varphi}\right)\right)\right| d \varphi \\
& -\ln \left|1+\sum_{n=2}^{+\infty} \frac{\cos (t \ln n)}{n^{2}}\right| .
\end{aligned}
$$

Если $|\pi-\varphi|>\arccos (3 /(2 R))$, то для $s=2 \pm i t+R e^{i \varphi}$ вешественная часть $s$ больше или равна 0.5 и выражение под знаком абсолютной величины в последнем интеграле не превосходит $0.5 \cdot 2(t+R)^{\varepsilon^{3}} \leqslant 2 t^{\varepsilon^{3}}$. Если $|\pi-\varphi| \leqslant \arccos (3 /(2 R))$, то

$$
2-R=0.5-\varepsilon^{2} \leqslant \operatorname{Re} s \leqslant 0.5 \text { и } 0 \leqslant 0.5-\operatorname{Re} s \leqslant \varepsilon^{2} .
$$

Из функционального уравнения дзета-функции для рассматриваемых $s$ получаем:

$$
\begin{aligned}
|\zeta(s)| & =\left|\pi^{s-1 / 2} \Gamma\left(\frac{s-1}{2}\right) \Gamma^{-1}\left(\frac{s}{2}\right)\right| \zeta(1-s) \mid \\
& =\left(\frac{t}{2 \pi}\right)^{0.5-\operatorname{Re} s}\left(1+O\left(t^{-1}\right)\right)|\zeta(1-s)| \leqslant 2 t^{\varepsilon^{2}+\varepsilon^{3}} \leqslant 2 t^{2 \varepsilon^{2}} .
\end{aligned}
$$

Таким образом,

$$
\begin{aligned}
\int_{0}^{R} \frac{n(r)}{r} d r & \leqslant \frac{1}{2 \pi} \cdot 2 \arccos \frac{3}{2 R} \cdot \ln \left(2 t^{2 \varepsilon^{2}}\right)+\ln \left(2 t^{2 \varepsilon^{3}}\right)-\ln \left(2-\frac{\pi^{2}}{6}\right) \\
& <\frac{4 \varepsilon}{2 \pi}\left(2 \varepsilon^{2} \ln t+1\right)+\left(\varepsilon^{3} \ln t+1\right)+2<2 \varepsilon^{3} \ln t
\end{aligned}
$$

$\mathrm{C}$ другой стороны,

$$
\int_{0}^{R} \frac{n(r)}{r} d r \geqslant \int_{1.5}^{R} \frac{n(r)}{r} d r \geqslant n(1.5) \int_{1.5}^{R} \frac{d r}{r}=n(1.5) \ln \frac{2 R}{3} \geqslant \frac{\varepsilon^{2}}{3} q .
$$

Таким образом, $q \leqslant 3 \varepsilon^{-2} 2 \varepsilon^{3} \ln t=6 \varepsilon \ln t$ и

$$
|S(t)|<7 \varepsilon \ln t .
$$

Пусть теперь $t$ совпадает с ординатой нуля $\zeta(s)$. Выберем $h$ с условием $0<h<\varepsilon$ так, чтобы интервал $(t, t+h)$ не содержал других ординат нулей $\zeta(s)$. По доказанному вьше, $|S(t+h)|<7 \varepsilon \ln (t+h)$. Поскольку на $(t, t+h) S(t)$ является гладкой функцией с производной $S^{\prime}(t)=-\frac{1}{2 \pi} \ln \frac{t}{2 \pi}+O\left(t^{-2}\right)$, то

и

$$
|S(t+h)-S(t)|=|S(t+h)-S(t+0)| \leqslant h\left(\frac{1}{2 \pi} \ln \frac{t}{2 \pi}+O\left(t^{-2}\right)\right)
$$

$$
|S(t)| \leqslant|S(t+h)|+h\left(\frac{1}{2 \pi} \ln \frac{t}{2 \pi}+O\left(t^{-2}\right)\right)<8 \varepsilon \ln t .
$$

Теорема доказана. 
Теорема 4. Если гипотеза Линделёфа верна, то $S_{1}(T)=o(\ln T)$.

ДоКАЗАТЕЛЬСТВО. Пусть $\varepsilon$ - сколь угодно малое фиксированное число, $0<\varepsilon<$ 0.1 . Покажем, что существуют $T_{0}=T_{0}(\varepsilon)>0$ и положительная абсолютная постоянная $a$ такие, что при $T>T_{0}$

$$
\left|S_{1}(T)\right| \leqslant a \varepsilon \ln T .
$$

Согласно лемме 8 гл. II,

$S_{1}(T)=\frac{1}{\pi} \int_{0.5}^{+\infty} \ln |\zeta(\sigma+i T)| d \sigma-\frac{1}{\pi} \int_{0.5}^{+\infty} \ln |\zeta(\sigma)| d \sigma=\frac{1}{\pi} \int_{0.5}^{2} \ln |\zeta(\sigma+i T)| d \sigma+O(1)$.

Положим $\delta=\varepsilon^{2}$ и представим последний интеграл суммою $I_{1}+I_{2}$, где

$$
I_{1}=\int_{0.5}^{0.5+3 \delta} \ln |\zeta(\sigma+i T)| d \sigma, \quad I_{2}=\int_{0.5+3 \delta}^{2} \ln |\zeta(\sigma+i T)| d \sigma .
$$

Интегрируя по $\sigma$ равенство

$$
\ln \zeta(s)=\sum_{|T-\gamma| \leqslant 1} \ln (s-\varrho)+O(\ln T)
$$

и отделяя в получившемся выражении вешественную часть, получим:

$$
I_{1}=\sum_{|T-\gamma| \leqslant 1} \int_{0.5}^{0.5+3 \delta} \ln |\zeta(\sigma+i T)| d \sigma+O(\delta \ln T) .
$$

Поскольку

$$
\ln |s-\varrho|=\frac{1}{2} \ln \left((\sigma-\beta)^{2}+(T-\gamma)^{2}\right)<\frac{1}{2} \ln \left(0.5^{2}+1\right)<1,
$$

TO

$$
I_{1}<\sum_{|T-\gamma| \leqslant 1} 3 \delta=O(\delta \ln T) .
$$

С другой стороны, так как $3 \delta<e^{-1}$, то

$$
\begin{aligned}
I_{1} & \geqslant \sum_{|T-\gamma| \leqslant 1} \int_{0.5}^{0.5+3 \delta} \ln |\sigma-\beta| d \sigma \geqslant 2 \sum_{|T-\gamma| \leqslant 1} \int_{0.5}^{0.5+3 \delta} \ln (0.5+3 \delta-\sigma) d \sigma \\
& >-\sum_{|T-\gamma| \leqslant 1} 5 \delta \ln \frac{1}{\delta}=-5 \delta \ln \frac{1}{\delta} \cdot O(\ln T) .
\end{aligned}
$$

Таким образом, $I_{1}=O\left(\delta \ln \left(\delta^{-1}\right) \cdot \ln T\right)=O(\varepsilon \ln T)$. Чтобы оценить интеграл $I_{2}$, положим

$$
\varepsilon_{1}=\exp \left(-\frac{2}{\varepsilon^{2}} \ln \frac{1}{\varepsilon}\right)
$$


Если гипотеза Линделёфа верна, то найдется такое $T_{1}(\varepsilon)$, что при $T \geqslant T_{1}(\varepsilon)>0$ справедливо неравенство:

$$
N\left(\frac{1}{2}+\delta, T+\frac{3}{2}\right)-N\left(\frac{1}{2}+\delta, T-\frac{3}{2}\right)<\varepsilon_{1} \ln T
$$

(см. [20; с. $327-328])$. Покажем, что на отрезке $0.5+3 \delta \leqslant \sigma \leqslant 2$ величина $\ln \zeta(\sigma+i T)$ приближается суммою $O\left(\varepsilon_{1} \ln T\right)$ слагаемых с точностью $O(\varepsilon \ln T)$. Пусть $C, C_{1}$ и $C_{2}-$ конщентрические круги с центром в точке $2+i T$ и радиусами $1.5-\delta, 1.5-2 \delta$ и $1.5-3 \delta$ соответственно. Положим

$$
\psi(s)=\ln \zeta(s)-\sum^{\prime} \ln (s-\varrho),
$$

где штрих означает суммирование по всем нулям $\varrho$, лежашим внутри и на границе $C$. Так как круг $C$ лежит правее прямой $\operatorname{Re} s=1 / 2+\delta$ и так как его радиус не превьшает $3 / 2$, то из приведенной вьше оценки разности $N\left(\frac{1}{2}+\delta, T+\frac{3}{2}\right)-N\left(\frac{1}{2}+\delta, T-\frac{3}{2}\right)$ следует, что сумма $\sum^{\prime}$ содержит $O\left(\varepsilon_{1} \ln T\right)$ слагаемых. Оценим сперва максимум $|\psi(s)|$ на границе круга $C_{1}$. Для этого заметим, что

$$
\psi(s)=-\sum_{|t-\gamma| \leqslant 1} \ln (s-\varrho)-\sum^{\prime} \ln (s-\varrho)+O(\ln t) .
$$

В это выражение для $\psi(s)$ входят слагаемые, отвечаюшие нулям $\varrho$ из круга $C$, для которых $|t-\gamma|>1$, а также нулям, удовлетворяюшим условию $|t-\gamma| \leqslant 1$, но лежашим вне $C$. Пусть $\varrho=\beta+i \gamma-$ один из таких нулей. Тогда для произвольной точки $s=\sigma+$ $i t$, лежащей на границе $C_{1}$, либо $|s-\varrho| \geqslant|t-\gamma|>1$, и вместе с тем

$$
|s-\varrho| \leqslant|\sigma-\beta|+|t-\gamma| \leqslant(1.5-\delta)+2(1.5-\delta)<4.5,
$$

либо $|s-\varrho| \geqslant \delta$, и вместе с тем

$$
|s-\varrho| \leqslant|\sigma-\beta|+|t-\gamma| \leqslant 2+(1.5-\delta)+1<5.5,
$$

так что $\ln |s-\varrho|=O\left(\ln \frac{1}{\delta}\right)$. Следовательно, на границе круга $C_{1}$

$$
|\psi(s)|=O\left(\left(\ln \frac{1}{\delta}\right) \ln T\right) .
$$

Пусть теперь $C_{3}-$ круг с центром в точке $2+i T$ и радиусом $1 / 2$. Так как для всякой точки $s$, лежашей внутри и на границе $C_{3}$,

$$
\ln \zeta(s)=O(1), \quad \ln (s-\varrho)=O(1) \quad \text { для каждого } \varrho \in C,
$$

то максимум $|\psi(s)|$ на границе $C_{3}$ есть

$$
O(1)+\sum^{\prime} O(1)=O\left(\varepsilon_{1} \ln T\right) .
$$


Обозначим буквой $M$ максимум $|\psi(s)|$ на границе круга $C_{2}$. По теореме Адамара о трех кругах (см. лемму 7П),

$$
M \ll\left(\varepsilon_{1} \ln T\right)^{\frac{\ln \left(r_{1} / r_{2}\right)}{\ln \left(r_{1} / r_{3}\right)}}\left(\left(\ln \frac{1}{\delta}\right) \ln T\right)^{\frac{\ln \left(r_{2} / r_{3}\right)}{\ln \left(r_{1} / r_{3}\right)}},
$$

где $r_{1}=1.5-2 \delta, r_{2}=1.5-3 \delta$ и $r_{3}=0.5$. Замечая, что

$\ln \frac{r_{1}}{r_{2}}=\ln (1-4 \delta / 3)-\ln (1-2 \delta)<\delta, \quad \ln \frac{r_{1}}{r_{3}}=\ln (3-4 \delta)>1, \quad \ln \frac{r_{2}}{r_{3}}=\ln (3-6 \delta)<\ln 3$, находим:

$$
M \ll \varepsilon_{1}^{\delta}\left(\ln \frac{1}{\delta}\right)^{\ln 3} \ln T \ll \varepsilon^{2}\left(\ln \frac{1}{\varepsilon^{2}}\right)^{\ln 3} \ln T \ll \varepsilon \ln T .
$$

Следовательно, внутри и на гранище $C_{2}$

$$
|\psi(s)|=O(\varepsilon \ln T) .
$$

Таким образом,

$$
\begin{aligned}
I_{2} & =\int_{0.5+3 \delta}^{2} \operatorname{Re}\left(\psi(\sigma+i T)+\sum^{\prime} \ln (\sigma+i T-\varrho)\right) d \sigma \\
& =O(\varepsilon \ln T)+\sum^{\prime} O(1)=O(\varepsilon \ln T)+O\left(\varepsilon_{1} \ln T\right)=O(\varepsilon \ln T) .
\end{aligned}
$$

Возврашаясь к оценке $S_{1}(T)$, находим:

$$
S_{1}(T)=\pi^{-1}\left(I_{1}+I_{2}\right)+O(1)=O(\varepsilon \ln T),
$$

где постоянная в знаке $O$ - абсолютная. Теорема доказана.

\section{§. Омега-теоремы}

Пусть функция $g(t)$ неотрицательна при достаточно больших $t$. Запись $f(t)=$ $\Omega(g(t))$ при $t \rightarrow+\infty$ означает существование бесконечной последовательности чисел $\left\{t_{j}\right\}, j=1,2, \ldots, t_{j} \rightarrow+\infty$, такой, что $f\left(t_{j}\right)>c g\left(t_{j}\right)$, где $c$ - некоторая постоянная.

Аналогично, равенство $f(t)=\Omega_{ \pm}(g(t))$ означает существование двух последовательностей $\left\{t_{j}\right\},\left\{\tau_{j}\right\}$, стремящихся к бесконечности и таких, что

$$
f\left(t_{j}\right)>c g\left(t_{j}\right), \quad f\left(\tau_{j}\right)<-c g\left(\tau_{j}\right), \quad j=1,2, \ldots .
$$

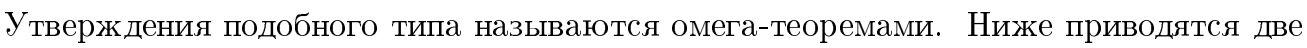
омега-теоремы для функции $S(t)$ - безусловная (теорема 5 ) и опираюшаяся на гипотезу Римана (теорема 6). Их доказательству предпошлем четыре вспомогательные леммы.

Лемма 1. Пусть $H>0, M>0, k \geqslant 1, k$ - целое число, и пусть вещественные функиии $W(t)$ и $R(t)$ удовлетворяют условиям

$$
\begin{aligned}
\int_{T}^{T+H}|W(t)|^{2 k} d t & \geqslant H M^{2 k}, \quad \text { 2) }\left|\int_{T}^{T+H}(W(t))^{2 k+1} d t\right| \leqslant 0.5 H M^{2 k+1}, \\
\text { 3) } & \int_{T}^{T+H}|R(t)|^{2 k+1} d t<H(0.5 M)^{2 k+1} .
\end{aligned}
$$

Тогда справедливы неравенства:

$$
\max _{T \leqslant t \leqslant T+H} \pm(W(t)+R(t)) \geqslant \frac{1}{8} M
$$


ДоКАЗАТЕЛЬСТВО. Положим для $r=0,1$

$$
W_{r}(t)=\max \left(0,(-1)^{r} W(t)\right)=\frac{1}{2}\left(|W(t)|+(-1)^{r} W(t)\right) .
$$

Достаточно показать, что

$$
\max _{T \leqslant t \leqslant T+H}\left(W_{r}(t)-|R(t)|\right) \geqslant \frac{1}{8} M .
$$

Действительно, если на промежутке $T \leqslant t \leqslant T+H$ найдутся точки $t_{0}$ и $t_{1}$, для которых $W_{r}\left(t_{r}\right)-\left|R\left(t_{r}\right)\right|>M / 8, r=0,1$, то $W_{r}\left(t_{r}\right)=(-1)^{r} W\left(t_{r}\right)>0$ и

$W\left(t_{0}\right)+R\left(t_{0}\right) \geqslant W\left(t_{0}\right)-\left|R\left(t_{0}\right)\right| \geqslant M / 8, \quad W\left(t_{1}\right)+R\left(t_{1}\right) \leqslant-W\left(t_{1}\right)+\left|R\left(t_{1}\right)\right| \leqslant-M / 8$.

Пусть $V(t)$ обозначает любую из функций $W_{r}(t)$, а $\mu$ - максимум разности $V(t)-|R(t)|$ на отрезке $T \leqslant t \leqslant T+H$. Определим числа $M_{1}$ и $M_{2}$ равенствами

$$
H^{-1} \int_{T}^{T+H} V^{m}(t) d t=M_{1}^{m}, \quad H^{-1} \int_{T}^{T+H}|R(t)|^{m} d t=M_{2}^{m}, \quad m=2 k+1 .
$$

Из условия леммы следует, что $M_{2}<0.5 M$. Чтобы оценить $M_{1}$ снизу, воспользуемся неравенством Гёльдера

$$
H M^{m-1} \leqslant \int_{T}^{T+H}|W(t)|^{m-1} d t \leqslant H^{1 / m}\left(\int_{T}^{T+H}|W(t)|^{m} d t\right)^{1-1 / m},
$$

из которого следует, что

$$
\int_{T}^{T+H}|W(t)|^{m} d t \geqslant H M^{m} .
$$

Пользуясь этой оценкой и определением величины $M_{1}$, находим:

$$
\begin{aligned}
H M_{1}^{m}=\int_{T}^{T+H}(V(t))^{m} d t & =\frac{1}{2}\left(\int_{T}^{T+H}|W(t)|^{m} d t+(-1)^{r} \int_{T}^{T+H}(W(t))^{m} d t\right) \\
& \geqslant 0.5\left(H M^{m}-0.5 H M^{m}\right)=0.25 H M^{m} \geqslant H\left(\frac{1}{\sqrt[3]{4}} M\right)^{m},
\end{aligned}
$$

т.е. $M_{1} \geqslant M / \sqrt[3]{4}$.

Рассмотрим теперь интеграл $j$,

$$
j=\int_{T}^{T+H}\left(V^{m}(t)-|R(t)|^{m}\right) d t .
$$

Из определения величин $M_{1}$ и $M_{2}$ мы заключаем, что

$$
j=H\left(M_{1}^{m}-M_{2}^{m}\right) \geqslant H\left(\left(\frac{1}{\sqrt[3]{4}} M\right)^{m}-\left(\frac{1}{2} M\right)^{m}\right)>0 .
$$


Разложим подьнтегральное выражение на множители:

$$
j=\int_{T}^{T+H}(V(t)-|R(t)|) \sum_{\nu=0}^{m-1} V^{\nu}(t)|R(t)|^{m-\nu-1} d t .
$$

Так как $j>0$, а каждое слагаемое в сумме по $\nu$ неотрицательно при любом $t$, то максимум $\mu$ величины $V(t)-|R(t)|$ положителен. Поэтому

$$
j \leqslant \mu \sum_{\nu=0}^{m-1} j(\nu), \text { где } j(\nu)=\int_{T}^{T+H} V^{\nu}(t)|R(t)|^{m-1-\nu} d t .
$$

$\mathrm{K}$ последнему интегралу дважды применим неравенство Гёльдера. Будем иметь:

$$
\begin{aligned}
j(\nu) & \leqslant\left(\int_{T}^{T+H} V^{m}(t) d t\right)^{\nu / m}\left(\int_{T}^{T+H}|R(t)|^{m(1-1 /(m-\nu))} d t\right)^{1-\nu / m} \\
& \leqslant\left(\int_{T}^{T+H} V^{m}(t) d t\right)^{\nu / m} H^{1 / m}\left(\int_{T}^{T+H}|R(t)|^{m} d t\right)^{(1-\nu / m)(1-1 /(m-\nu))} \\
& =H M_{1}^{\nu} M_{2}^{m-\nu-1} .
\end{aligned}
$$

Таким образом,

$$
H\left(M_{1}^{m}-M_{2}^{m}\right)=j \leqslant \mu \sum_{\nu=0}^{m-1} H M_{1}^{\nu} M_{2}^{m-\nu-1}=\mu H \frac{M_{1}^{m}-M_{2}^{m}}{M_{1}-M_{2}} .
$$

Сокрашая обе части этого неравенства на $H\left(M_{1}^{m}-M_{2}^{m}\right)$, находим:

$$
\mu \geqslant M_{1}-M_{2} \geqslant\left(\frac{1}{\sqrt[3]{4}}-\frac{1}{2}\right) M \geqslant \frac{1}{8} M .
$$

Лемма доказана.

СлЕДСТвИЕ. Пусть $H>0, M>0, k \geqslant 1, k$ - иелое число, и пусть вещественная непрерывная функиия $W(t)$ удовлетворяет неравенствам 1) и 2) из условия леммы. Тогда справедливы соотношения:

$$
\max _{T \leqslant t \leqslant T+H} \pm W(t) \geqslant \frac{1}{8} M .
$$

Лемма 2. Для любых достаточно больших положительных $H, t u \tau, \tau<\ln t$, $H<t$, справедлива форммла:

$$
\int_{-0.5 H \tau}^{0.5 H \tau}\left(\frac{\sin u}{u}\right)^{2} S\left(t+\frac{2 u}{\tau}\right) d u=W(t)+R(t)+O\left(\ln \tau+\frac{\ln t}{H \tau}\right),
$$

в которой

$$
\begin{aligned}
W(t) & =-\sum_{p \leqslant e^{\tau}} \frac{\sin (t \ln p)}{\sqrt{p}}\left(1-\frac{\ln p}{\tau}\right), \\
R(t) & =\tau \sum_{\beta>0.5} \int_{0}^{\beta-0.5} \operatorname{Im}\left(\frac{\sin \frac{\tau}{2}(\gamma-t-i \xi)}{\frac{\tau}{2}(\gamma-t-i \xi)}\right)^{2} d \xi,
\end{aligned}
$$

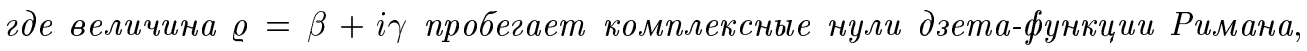
лежащие правее критической прямой. 
ДокАЗАТЕЛЬСТво. Полагая

$$
f(z)=\left(\frac{\sin (\tau z / 2)}{\tau z / 2}\right)^{2}
$$

будем иметь

$$
\widehat{f}(x)=\int_{-\infty}^{+\infty} e^{-i x u} f(u) d u=\frac{2 \pi}{\tau} \max (0,1-|x / \tau|),
$$

так что

$$
\widehat{f}(\ln n)= \begin{cases}\frac{2 \pi}{\tau}\left(1-\frac{\ln n}{\tau}\right), & \text { если } 1 \leqslant n \leqslant e^{\tau}, \\ 0, & \text { если } n>e^{\tau} .\end{cases}
$$

Воспользовавшись следствием теоремы 3 гл. II, получим:

$$
\begin{aligned}
& \int_{-\infty}^{+\infty}\left(\frac{\sin (\tau u / 2)}{\tau u / 2}\right)^{2} S(t+u) d u=-\frac{2}{\tau} \sum_{n \leqslant e^{\tau}} \frac{\Lambda(n)}{\ln n} \frac{\sin (t \ln n)}{\sqrt{n}}\left(1-\frac{\ln n}{\tau}\right) \\
& \quad+2\left(\sum_{\beta>0.5} \int_{0}^{\beta-0.5} \operatorname{Im}\left(\frac{\sin \frac{\tau}{2}(\gamma-t-i \xi)}{\frac{\tau}{2}(\gamma-t-i \xi)}\right)^{2} d \xi-\int_{0}^{0.5} \operatorname{Im}\left(\frac{\sin \frac{\tau}{2}(t+i \xi)}{\frac{\tau}{2}(t+i \xi)}\right)^{2} d \xi\right) .
\end{aligned}
$$

Так как при $0 \leqslant \xi \leqslant 0.5$

$$
2\left|\frac{\sin \frac{\tau}{2}(t+i \xi)}{\frac{\tau}{2}(t+i \xi)}\right|^{2} \leqslant 2\left(\frac{2 e^{\tau \xi / 2}}{t}\right)^{2} \leqslant 2\left(\frac{2 e^{\tau / 4}}{t}\right)^{2}<8 e^{0.5 \ln t} t^{-2}<0.2 \tau^{-1},
$$

то последний интеграл по абсолютной величине не превосходит $0.1 \tau^{-1}$.

В сумме по $n \leqslant e^{\tau}$ выделим слагаемые, отвечаюшие простым $n=p$ и оценим оставшиеся по модулю:

$$
\begin{aligned}
& \frac{2}{\tau}\left|\sum_{k \geqslant 2} \sum_{n=p^{k} \leqslant e^{\tau}} \frac{\Lambda(n)}{\ln n} \frac{\sin (t \ln n)}{\sqrt{n}}\left(1-\frac{\ln n}{\tau}\right)\right| \leqslant \frac{1}{\tau} \sum_{p \leqslant e^{\tau / 2}} \sum_{k \geqslant 2} p^{-k / 2} \\
& <\frac{2}{\tau} \sum_{p \leqslant e^{\tau / 2}} \frac{1}{p}=\frac{2}{\tau}\left(\ln \frac{\tau}{2}+0.26 \ldots+O\left(\tau^{-1}\right)\right)<(2 \ln \tau-0.1) \tau^{-1} .
\end{aligned}
$$

Таким образом,

$$
\begin{aligned}
& \int_{-\infty}^{+\infty}\left(\frac{\sin (\tau u / 2)}{\tau u / 2}\right)^{2} S(t+u) d u=-\frac{2}{\tau} \sum_{p \leqslant e^{\tau}} \frac{\sin (t \ln p)}{\sqrt{p}}\left(1-\frac{\ln p}{\tau}\right) \\
& \quad+2 \sum_{\beta>0.5} \int_{0}^{\beta-0.5} \operatorname{Im}\left(\frac{\sin \frac{\tau}{2}(\gamma-t-i \xi)}{\frac{\tau}{2}(\gamma-t-i \xi)}\right)^{2} d \xi+\theta \frac{2}{\tau} \ln \tau .
\end{aligned}
$$

Делая в несобственном интеграле замену $v=\tau u / 2$ и разбивая прямую интегрирования на три промежутка, представим левую часть равенства суммою

$$
\frac{2}{\tau}\left(\int_{-0.5 H \tau}^{0.5 H \tau}+\int_{-\infty}^{-0.5 H \tau}+\int_{0.5 H \tau}^{+\infty}\right)\left(\frac{\sin v}{v}\right)^{2} S\left(t+\frac{2 v}{\tau}\right) d v
$$


и оценим два последних слагаемых, пользуясь теоремой 1 настоящей главы:

$$
\begin{aligned}
& \left|2 \tau\left(\int_{-\infty}^{-0.5 H \tau}+\int_{0.5 H \tau}^{+\infty}\right)\left(\frac{\sin v}{v}\right)^{2} S\left(t+\frac{2 v}{\tau}\right) d v\right| \\
& \quad \ll \tau \cdot \tau^{-1} \int_{H}^{+\infty} \ln (t+v) \frac{d v}{v^{2}} \ll \int_{H}^{t} \ln t \frac{d v}{v^{2}}+\int_{t}^{+\infty} \ln v \frac{d v}{v^{2}} \ll \frac{\ln t}{H}+\frac{\ln t}{t} \ll \frac{\ln t}{H} .
\end{aligned}
$$

Сокрашая обе части рассматриваемого соотношения на $2 / \tau$, получим:

$$
\int_{-0.5 H \tau}^{0.5 H \tau}\left(\frac{\sin u}{u}\right)^{2} S\left(t+\frac{2 u}{\tau}\right) d u=W(t)+R(t)+O\left(\ln \tau+\frac{\ln t}{H \tau}\right)
$$

где

$$
\begin{aligned}
& W(t)=-\sum_{p \leqslant e^{\tau}} \frac{\sin (t \ln p)}{\sqrt{p}}\left(1-\frac{\ln p}{\tau}\right) \\
& R(t)=\tau \sum_{\beta>0.5} \int_{0}^{\beta-0.5} \operatorname{Im}\left(\frac{\sin \frac{\tau}{2}(\gamma-t-i \xi)}{\frac{\tau}{2}(\gamma-t-i \xi)}\right)^{2} d \xi
\end{aligned}
$$

Лемма доказана.

Лемма 3. Пусть $T \geqslant T_{0}>0, e^{2}<H<T, 2<\tau<\ln H, k \geqslant k_{0}>1, k$ - иелое число, $2 k \ln k<e^{2 \tau / 3}$. Тогда справедливы неравенства:

$$
\int_{T}^{T+H}(W(t))^{2 k} d t>(0.0025 k)^{k} H-e^{3 k \tau}, \quad\left|\int_{T}^{T+H}(W(t))^{2 k+1} d t\right|<e^{3 k \tau} e^{1.5 \tau}
$$

ДокАЗАТЕЛЬСТво. Представляя $W(t)$ в виде разности $(V(t)-\bar{V}(t)) /(2 i)$,

$$
V(t)=\sum_{p \leqslant e^{\tau}} \frac{a(p)}{\sqrt{p}} p^{-i t}, \quad a(p)=1-\frac{\ln p}{\tau},
$$

найдем:

$$
\begin{aligned}
& I_{k}=\int_{T}^{T+H}(W(t))^{2 k} d t=(2 i)^{-2 k} \sum_{\nu=0}^{2 k}(-1)^{\nu}\left(\begin{array}{c}
2 k \\
\nu
\end{array}\right) j_{2 k}(\nu), \\
& J_{k}=\int_{T}^{T+H}(W(t))^{2 k+1} d t=(2 i)^{-2 k-1} \sum_{\nu=0}^{2 k+1}(-1)^{\nu}\left(\begin{array}{c}
2 k+1 \\
\nu
\end{array}\right) j_{2 k+1}(\nu),
\end{aligned}
$$

где

$$
\begin{aligned}
j_{r}(\nu) & =\int_{T}^{T+H} V^{\nu}(t) \bar{V}^{r-\nu}(t) d t \\
& =\sum_{p_{1}, \ldots, p_{\nu} \leqslant e^{\tau}} \sum_{q_{1}, \ldots, q_{r-\nu} \leqslant e^{\tau}} \frac{a\left(p_{1}\right) \cdots a\left(q_{r-\nu}\right)}{\sqrt{p_{1} \cdots q_{r-\nu}}} \int_{T}^{T+H}\left(\frac{q_{1} \cdots q_{r-\nu}}{p_{1} \cdots p_{\nu}}\right)^{i t} d t .
\end{aligned}
$$


Из основной теоремы арифметики следует, что равенство $q_{1} \cdots q_{r-\nu}=p_{1} \cdots p_{\nu}$ возможно лишш в случае, когда $r=2 k, \nu=k$. В остальных случаях, полагая для краткости $P=p_{1} \cdots p_{\nu}, Q=q_{1} \cdots q_{r-\nu}$ и замечая, что $P$ и $Q$ не превосходят $e^{r \tau}$, находим:

$$
\begin{aligned}
\left|j_{r}(\nu)\right| & \leqslant \sum_{P \neq Q} \frac{1}{\sqrt{P Q}} \cdot 2\left|\ln \frac{Q}{P}\right|^{-1} \leqslant 2 \sum_{P, Q} \frac{1}{\sqrt{P Q}} \max (P, Q) \\
& \leqslant 2 e^{r \tau} \sum_{P, Q} \frac{1}{\sqrt{P Q}} \leqslant 2 e^{r \tau}\left(\sum_{p \leqslant e^{\tau}} \frac{1}{\sqrt{p}}\right)^{r} \leqslant 2 e^{r \tau}\left(3 \tau^{-1} e^{\tau / 2}\right)^{r}<e^{1.5 r \tau}
\end{aligned}
$$

В случае $r=2 k, \nu=k$ в выражении для $j_{r}(\nu)$ присутствуют “диагональные" члены:

$$
j_{2 k}(k)=H \Sigma+\theta \sum_{P \neq Q} \frac{1}{\sqrt{P Q}} \cdot 2\left|\ln \frac{Q}{P}\right|^{-1}=H \Sigma+\theta e^{1.5 \cdot 2 k \tau}
$$

где $|\theta|<1$, a

$$
\Sigma=\sum_{p_{1} \cdots p_{k}=q_{1} \cdots q_{k}} \frac{a\left(p_{1}\right) \cdots a\left(q_{k}\right)}{\sqrt{p_{1} \cdots q_{k}}}=\sum_{p_{1} \cdots p_{k}=q_{1} \cdots q_{k}} \frac{a^{2}\left(p_{1}\right) \cdots a^{2}\left(p_{k}\right)}{p_{1} \cdots p_{k}}
$$

Оставляя в последней сумме слагаемые, отвечаюшие наборам $\left(p_{1}, \ldots, p_{k}\right)$, не содержашим повторений, придем к нижней оценке величины $\Sigma$. Зафиксируем набор $\left(p_{1}, \ldots, p_{k}\right)$, в котором все числа $p_{1}, \ldots, p_{k}$ различны. Тогда число решений уравнения $p_{1} \cdots p_{k}=q_{1} \cdots q_{k}$ в простых числах $q_{1}, \ldots, q_{k}$ равно $k !$, и поэтому

$$
\Sigma \geqslant k ! \sum_{p_{1} \leqslant e^{\tau}} \frac{a^{2}\left(p_{1}\right)}{p_{1}} \sum_{\substack{p_{2} \leqslant e^{\tau} \\ p_{2} \neq p_{1}}} \frac{a^{2}\left(p_{2}\right)}{p_{2}} \sum_{\substack{p_{3} \leqslant e^{\tau} \\ p_{3} \neq p_{1}, p_{2}}} \frac{a^{2}\left(p_{3}\right)}{p_{3}} \cdots \sum_{\substack{p_{k} \leqslant e^{\tau} \\ p_{k} \neq p_{1}, \ldots, p_{k-1}}} \frac{a^{2}\left(p_{k}\right)}{p_{k}}
$$

Величины $a^{2}(p) p^{-1}$ с ростом $p, p \leqslant e^{\tau}$, монотонно убывают, так как

$$
\frac{d}{d p}\left(\frac{a^{2}(p)}{p}\right)=\frac{d}{d p}\left(\frac{1}{p}\left(1-\frac{\ln p}{\tau}\right)^{2}\right)=-\frac{1}{p^{2}}\left(1-\frac{\ln p}{\tau}\right)\left(1-\frac{\ln p}{\tau}+\frac{2}{\tau}\right)<0
$$

Следовательно, при любых значениях $p_{1}, \ldots, p_{k-1}$ сумма по $p_{k}$ не меньше суммы величин $a^{2}(p) p^{-1}$ по простым числам $p \leqslant e^{\tau}$, из которой выброшены слагаемые, отвечаюшие первым $k-1$ простым числам. Считая $k$ настолько большим, что $(k-1)$-е простое число не превосходит $2 k \ln k$, убеждаемся, что сумма по $p_{k}$ оценивается снизу величиной

$$
\sum_{e^{2 \tau / 3}<p \leqslant e^{\tau}} \frac{a^{2}(p)}{p}
$$


которая не зависит от $p_{1}, \ldots, p_{k-1}$. Применяя те же рассуждения к сумме по $p_{k-1}$, затем к суммам по $p_{k-2}, \ldots, p_{1}$, получим:

$$
\begin{aligned}
\Sigma & \geqslant k !\left(\sum_{e^{2 \tau / 3}<p \leqslant e^{\tau}} \frac{a^{2}(p)}{p}\right)^{k}>k !\left(\sum_{e^{2 \tau / 3}<p \leqslant e^{4 \tau / 5}} \frac{1}{p}\left(1-\frac{\ln p}{\tau}\right)^{2}\right)^{k} \\
& >k !\left(\sum_{e^{2 \tau / 3}<p \leqslant e^{4 \tau / 5}} \frac{1}{5^{2} p}\right)^{k}=5^{-2 k} k !\left(\ln \frac{4}{5} \tau-\ln \frac{2}{3} \tau+o(1)\right)^{k} \\
& >(0.18)^{k} 5^{-2 k} k !>(0.007)^{k} k ! .
\end{aligned}
$$

Итак,

$$
\begin{gathered}
j_{2 k}(k)>(0.007)^{k} k !-e^{3 k \tau} \\
I_{k}>2^{-2 k}\left(\begin{array}{c}
2 k \\
k
\end{array}\right)(0.007)^{k} k ! H-e^{3 k \tau} 2^{-2 k} \sum_{\nu=0}^{2 k}\left(\begin{array}{c}
2 k \\
\nu
\end{array}\right) \\
>\left(0.007 e^{-1} k\right)^{k} H-e^{3 k \tau}>(0.0025 k)^{k} H \\
\left|J_{k}\right|<2^{-2 k-1} e^{1.5(2 k+1) \tau} \sum_{\nu=0}^{2 k+1}\left(\begin{array}{c}
2 k+1 \\
\nu
\end{array}\right)=e^{3 k \tau} e^{1.5 \tau} .
\end{gathered}
$$

Лемма доказана.

Для доказательства следующей леммы нам потребуется утверждение, которое назовем "плотностная теорема".

ПЛотностная тЕОРемА. При фиксированном $\varepsilon, 0<\varepsilon<0.001, T \geqslant T_{0}(\varepsilon)>0$, $H=T^{27 / 82+\varepsilon}$ u $0.5 \leqslant \sigma \leqslant 1$ выполняется оценка

$$
N(\sigma, T+H)-N(\sigma, T-H) \leqslant 0.4 H(\ln T) T^{-0.1 \varepsilon(2 \sigma-1)} .
$$

Доказательство этого утверждения см. в [21], [22].

Лемма 4. Пусть $\varepsilon-$ произвольное фиксированное число, $0<\varepsilon<0.001, T \geqslant$ $T_{0}(\varepsilon)>0, H=T^{27 / 82+\varepsilon}, k \geqslant k_{0}(\varepsilon)>1, k-$ челое число, $m=2 k+1, \tau=2 \ln \ln H$, и пусть $m \tau<0.1 \varepsilon \ln T$. Тогда для определенной выце функиии $R(t)$ справедливо следующее неравенство:

$$
\int_{T}^{T+H}|R(t)|^{m} d t<H\left(50^{m}+(\ln T)^{3}\left(\frac{100 \varepsilon^{-3} \tau m^{2}}{\ln T}\right)^{m}\right) .
$$

ДокАЗАТЕльСТво. Перед тем как заняться непосредственно оценкой интеграла

$$
L_{k}=\int_{T}^{T+H}|R(t)|^{2 k+1} d t,
$$


получим необходимую для дальнейшего верхнюю границу подьнтегральной функции. Для этого заметим, что при любых вешественных $x$ и $y, y \geqslant 0$, справедливо неравенство:

$$
\left|\operatorname{Im}\left(\frac{\sin (x-i y)}{x-i y}\right)^{2}\right|<\frac{32 y e^{2 y}}{1+x^{2}+y^{2}} .
$$

Действительно, обозначив левую часть неравенства символом $\varphi(x, y)$, представим ее в виде

$$
\varphi=\left|\operatorname{Im}\left(\left(\frac{\sin (x-i y)}{x-i y}\right)^{2}-\left(\frac{\sin x}{x}\right)^{2}\right)\right|=2\left|\operatorname{Im} \int_{x}^{x-i y} \frac{\sin z}{z} \cdot \frac{z \cos z-\sin z}{z^{2}} d z\right|,
$$

где $z=x-i v, 0 \leqslant v \leqslant y$. Если $|z| \leqslant 1$, то

$$
\begin{aligned}
\left|\frac{\sin z}{z}\right| & =\left|1-\frac{z^{2}}{3 !}+\frac{z^{4}}{5 !}-\cdots\right|<\frac{6}{5}<\frac{6 \sqrt{2}}{5} \frac{e^{v}}{\sqrt{1+x^{2}+v^{2}}}, \\
\left|\frac{z \cos z-\sin z}{z^{2}}\right| & =\left|\left(\frac{1}{2 !}-\frac{1}{3 !}\right) z-\left(\frac{1}{4 !}-\frac{1}{5 !}\right) z^{3}+\left(\frac{1}{6 !}-\frac{1}{7 !}\right) z^{5}-\cdots\right|<\frac{3}{4} \\
& <\frac{3 \sqrt{2}}{4} \frac{e^{v}}{\sqrt{1+x^{2}+v^{2}}} .
\end{aligned}
$$

Если $|z|>1$, то

$$
\left|\frac{\sin z}{z}\right| \leqslant \frac{e^{v}}{|z|} \leqslant \sqrt{2} \frac{e^{v}}{\sqrt{1+x^{2}+v^{2}}}, \quad\left|\frac{z \cos z-\sin z}{z^{2}}\right| \leqslant \frac{e^{v}}{|z|} \leqslant 2 \sqrt{2} \frac{e^{v}}{\sqrt{1+x^{2}+v^{2}}} .
$$

В каждом из случаев подьнтегральная функция в выражении для $\varphi(x, y)$ по абсолютной величине не превосходит

$$
\frac{4 e^{v}}{\sqrt{1+x^{2}+v^{2}}} \text {. }
$$

При фиксированном $x$ эта величина - неубьвающая функция $v$, так что

$$
\varphi(x, y) \leqslant 8 \int_{0}^{y} \frac{e^{2 y}}{1+x^{2}+y^{2}} d v=\frac{8 y e^{2 y}}{1+x^{2}+y^{2}} .
$$

Пользуясь этой оценкой наряду с определением $R(t)$, находим:

$$
\begin{aligned}
|R(t)| & \leqslant \tau\left|\sum_{\beta>0.5} \int_{0}^{\beta-0.5} \operatorname{Im}\left(\frac{\sin \frac{\tau}{2}(\gamma-t-i \xi)}{\frac{\tau}{2}(\gamma-t-i \xi)}\right)^{2} d \xi\right| \\
& \leqslant \tau \sum_{\beta>0.5} \int_{0}^{\beta-0.5} \frac{8 \frac{\tau \xi}{2} e^{\tau \xi} d \xi}{1+\left(\frac{\tau}{2}(\gamma-t)\right)^{2}+\left(\frac{\tau \xi}{2}\right)^{2}} \\
& <4 \tau^{2} \sum_{\beta>0.5} \int_{0}^{\beta-0.5} \frac{\xi e^{\tau(\beta-0.5)} d \xi}{1+\left(\frac{\tau}{2}(\gamma-t)\right)^{2}+\left(\frac{\tau}{2}(\beta-0.5)\right)^{2}} \\
& =8 \sum_{\beta>0.5}(\beta-0.5)^{2} \frac{e^{\tau(\beta-0.5)}}{\left(\frac{2}{\tau}\right)^{2}+(\gamma-t)^{2}+(\beta-0.5)^{2}} .
\end{aligned}
$$


Сумму по нулям разобьем на две, относя к первой слагаемые, отвечающие условию $|\gamma-t|>(\ln T)^{2}$, а ко второй - все остальные. Пользуясь неравенством теоремы 1, мы заключаем, что

$$
N(t+1)-N(t) \leqslant \frac{1}{2 \pi} \ln \frac{t+1}{2 \pi}+|S(t+1)|+|S(t)|+O\left(t^{-1}\right)<18 \ln t .
$$

Поэтому первая сумма не превосходит

$$
\begin{aligned}
2 e^{\tau / 2} \sum_{|\gamma-t|>(\ln T)^{2}}(\gamma-t)^{-2} & <\frac{2}{3}(\ln T) \sum_{n>(\ln T)^{2}} n^{-2} \sum_{n<|\gamma-t| \leqslant n+1} 1 \\
& <\frac{2}{3}(\ln T) \cdot 36 \sum_{n>(\ln T)^{2}}(\ln T+\ln n) n^{-2}<25 .
\end{aligned}
$$

Следуя А. Сельбергу [27], обозначим символом $\theta_{t}$ наибольшую из разностей $\beta-0.5$ для нулей $\varrho=\beta+i \gamma$, лежаших в прямоугольнике $0.5<\beta \leqslant 1,|\gamma-t| \leqslant(\ln T)^{2}$. Тогда вторая сумма не превзойдет величины

$$
8 \theta_{t}^{2} e^{\tau \theta_{t}} \sum_{|\gamma-t| \leqslant(\ln T)^{2}}\left(\left(\frac{2}{\tau}\right)^{2}+(\gamma-t)^{2}+(\beta-0.5)^{2}\right)^{-1} .
$$

Положим $x=\sqrt{\ln T}$. Из определения величин $\sigma_{x, t}, \theta_{t}$ и $\tau$ следует, что

$$
\sigma_{x, t}-0.5 \leqslant 2 \max \left(\theta_{t}, \frac{4}{\tau}\right) \leqslant 4\left(\frac{2}{\tau}+\theta_{t}\right)
$$

и

$$
\left(\frac{2}{\tau}\right)^{2}+\theta_{t}^{2} \geqslant\left(\frac{2}{\tau}+\theta_{t}\right)^{2} \geqslant \frac{1}{32}\left(\sigma_{x, t}-0.5\right)^{2} \geqslant \frac{1}{32}\left(\sigma_{x, t}-\beta\right)^{2} .
$$

Таким образом, верхней границей рассматриваемой суммы будет

$$
2^{8} \theta_{t}^{2} e^{\tau \theta_{t}} \sum_{\varrho}\left(\left(\sigma_{x, t}-\beta\right)^{2}+(\gamma-t)^{2}\right) .
$$

Согласно следствию леммы 7 гл. II, последняя сумма не превосходит

$$
\frac{13}{3}\left(\sigma_{x, t}-0.5\right)^{-1}\left(\sum_{n \leqslant(\ln T)^{1.5}} \frac{\ln n}{\sqrt{n}}+0.5 \ln (T+H)+4\right)<\frac{13}{3}\left(\sigma_{x, t}-0.5\right)^{-1} \cdot 0.6 \ln T .
$$

Поскольку

TO

$$
\sigma_{x, t}-0.5 \geqslant \frac{2}{\ln x}=\frac{4}{\ln \ln T} \geqslant \frac{3.9}{\ln \ln H}=\frac{7.8}{\tau},
$$

$$
|R(t)|<25+2^{8} \theta_{t}^{2} e^{\tau \theta_{t}} \frac{13}{3} \cdot \frac{\tau}{7.8} \cdot 0.6 \ln T<25\left(1+3.5 \theta_{t}^{2} e^{\tau \theta_{t}} \tau \ln T\right) .
$$


Приступим к оценке интеграла $L_{k}$. Полагая $m=2 k+1$ и пользуясь неравенством Гёльдера, будем иметь:

$$
L_{k}<25^{m} \int_{T}^{T+H}\left(1+3.5 \theta_{t}^{2} e^{\tau \theta_{t}} \tau \ln T\right)^{m} d t<50^{m}\left(H+(3.5 \tau \ln T)^{m} j\right)
$$

где обозначено:

$$
j=\int_{T}^{T+H}\left(\theta_{t}^{2} e^{\tau \theta_{t}}\right)^{m} d t
$$

Пусть $\beta_{0}+i \gamma_{0}-$ произвольный нуль $\zeta(s)$ с условием $T-(\ln T)^{2} \leqslant \gamma \leqslant T+H+(\ln T)^{2}$. Из определения $\theta_{t}$ следует, что множество тех $t \in(T, T+H]$, для которых $\theta_{t}=\beta_{0}-0.5$, покрыто промежутком $\gamma-(\ln T)^{2} \leqslant t \leqslant \gamma+(\ln T)^{2}$ (возможно, окажется пустьм). Поэтому

$$
\begin{aligned}
j & \leqslant \sum_{T-(\ln T)^{2} \leqslant \gamma \leqslant T+H+(\ln T)^{2}} \int_{\gamma-(\ln T)^{2}}^{\gamma+(\ln T)^{2}}(\beta-0.5)^{2 m} X^{\beta-0.5} d t \\
& \leqslant 2(\ln T)^{2} \sum_{T-H<\gamma \leqslant T+2 H}(\beta-0.5)^{2 m} X^{\beta-0.5},
\end{aligned}
$$

где $X=e^{m \tau}$. Представляя величину $(\beta-0.5)^{2 m} X^{\beta-0.5}$ интегралом

$$
\begin{gathered}
\int_{0}^{\beta-0.5}\left(2 m u^{2 m-1}+m \tau u^{2 m}\right) X^{u} d u=\int_{0}^{0.5} g(\varrho ; u)\left(2 m u^{2 m-1}+m \tau u^{2 m}\right) X^{u} d u, \\
g(\varrho ; u)=g(\beta+i \gamma ; u)= \begin{cases}1, & \text { если } 0 \leqslant u \leqslant \beta-0.5, \\
0, & \text { если } \beta-0.5<u \leqslant 0.5,\end{cases}
\end{gathered}
$$

получим:

$$
\begin{aligned}
j \leqslant & 2(\ln T)^{2} \int_{0}^{0.5}\left(\sum_{T-H<\gamma \leqslant T+2 H} g(\varrho ; u)\right)\left(2 m u^{2 m-1}+m \tau u^{2 m}\right) X^{u} d u \\
= & 2(\ln T)^{2} \int_{0}^{0.5}(N(0.5+u, T+2 H)-N(0.5+u, T-H)) \\
& \times X^{u}\left(2 m u^{2 m-1}+m \tau u^{2 m}\right) d u .
\end{aligned}
$$

Пользуясь неравенством плотностной теоремы и тем, что $X<e^{0.1 \varepsilon \ln T}=T^{0.1 \varepsilon}$, находим:

$$
\begin{aligned}
j & \leqslant 2.4 H(\ln T)^{3} \int_{0}^{0.5}\left(T^{-0.2 \varepsilon} X\right)^{u}\left(2 m u^{2 m-1}+m \tau u^{2 m}\right) d u \\
& <2.4 H(\ln T)^{3} \int_{0}^{0.5} T^{-0.1 u \varepsilon}\left(2 m u^{2 m-1}+m \tau u^{2 m}\right) d u .
\end{aligned}
$$


Положим в последнем интеграле $v=0.1 u \varepsilon \ln T$. Тогда

$$
\begin{aligned}
j< & 2.4 H(\ln T)^{3}\left(2 m(0.1 \varepsilon \ln T)^{-2 m} \int_{0}^{+\infty} v^{2 m-1} e^{-v} d v\right. \\
& \left.+m \tau(0.1 \varepsilon \ln T)^{-2 m-1} \int_{0}^{+\infty} v^{2 m} e^{-v} d v\right) \\
< & 2.4 H(\ln T)^{3}(2 m) !(0.1 \varepsilon \ln T)^{-2 m}\left(\frac{1}{m}+\frac{\tau}{0.1 \varepsilon \ln T}\right) \\
< & 4.8 m^{-1} H(\ln T)^{3}(2 m) !(0.1 \varepsilon \ln T)^{-2 m}
\end{aligned}
$$

и, следовательно,

$$
\begin{aligned}
L_{k} & <50^{m} H\left(1+4.8 m^{-1}(\ln T)^{3}(2 m) !(3.5 \tau \ln T)^{m}(0.1 \varepsilon \ln T)^{-2 m}\right) \\
& \leqslant 50^{m} H\left(1+4.8 m^{-1}(\ln T)^{3}\left(\frac{3500 \varepsilon^{-2} \tau}{\ln T}\right) \cdot 3.6 \sqrt{m}\left(\frac{2 m}{e}\right)^{2 m}\right) \\
& <50^{m} H\left(1+18 m^{-0.5}(\ln T)^{3}\left(\frac{2 \cdot 10^{3} \varepsilon^{-2} \tau m^{2}}{\ln T}\right)^{m}\right) \\
& <50^{m} H\left(1+(\ln T)^{3}\left(\frac{2 \varepsilon^{-3} \tau m^{2}}{\ln T}\right)^{m}\right) \\
& <H\left(50^{m}+(\ln T)^{3}\left(\frac{100 \varepsilon^{-3} \tau m^{2}}{\ln T}\right)^{m}\right) .
\end{aligned}
$$

Лемма доказана.

ТеОРема 5. Пусть $\varepsilon-$ произвольное фиксированное число, $0<\varepsilon<10^{-7}, T \geqslant$ $T_{0}(\varepsilon)>0, H=T^{27 / 82+\varepsilon}$. Тогда справедливы неравенства:

$$
\sup _{T-H \leqslant t \leqslant T+2 H}( \pm S(t)) \geqslant \frac{\varepsilon^{5 / 4}}{1000}\left(\frac{\ln T}{\ln \ln T}\right)^{1 / 3}
$$

ДОКАЗАТЕЛЬСТВО. ПолОЖим

$$
k=\left[\frac{\varepsilon^{2.5}}{10}\left(\frac{\ln T}{\ln \ln T}\right)^{2 / 3}\right], \quad \tau=2 \ln \ln H .
$$

Тогда будут выполнены условия

$$
\begin{gathered}
2 k \ln k<(2 k+1) \tau<0.1 \varepsilon \ln T<e^{2 \tau / 3}=(\ln H)^{4 / 3}, \\
e^{3 k \tau}<\exp \left(6\left(\frac{\varepsilon^{2.5}}{10}\left(\frac{\ln T}{\ln \ln T}\right)^{2 / 3}+1\right) \ln \ln T\right)<\sqrt{H}
\end{gathered}
$$


и, как следствие, оценки интегралов $I_{k}, J_{k}$ и $L_{k}$ лемм 3 и 4 :

$$
\begin{aligned}
I_{k}> & (0.0025 k)^{k} H-e^{3 k \tau}>(0.05 \sqrt{k})^{2 k} H-\sqrt{H}>(0.04 \sqrt{k})^{2 k} H, \\
& \left|J_{k}\right|<e^{3 k \tau} e^{1.5 \tau}<\sqrt{H}(\ln H)^{3}<0.5 H(0.04 \sqrt{k})^{2 k+1}, \\
L_{k}< & H\left(50^{2 k+1}+(\ln T)^{3}\left(\frac{100 \varepsilon^{-3} \cdot 5 k^{2} \tau}{\ln T}\right)^{2 k+1}\right) \\
< & H\left(50^{2 k+1}+(\ln T)^{3}\left(10 \varepsilon^{2}\left(\frac{\ln T}{\ln \ln T}\right)^{1 / 3}\right)^{2 k+1}\right) \\
< & H\left(0.5(0.02 \sqrt{k})^{2 k+1}+\left(60 \varepsilon^{1 / 4}\right)^{2 k+1}(\ln T)^{3} \cdot\left(\varepsilon^{1 / 4} \sqrt{k}\right)^{2 k+1}\right) \\
< & H\left(0.5(0.02 \sqrt{k})^{2 k+1}+0.5(0.02 \sqrt{k})^{2 k+1}\right)=(0.02 \sqrt{k})^{2 k+1} H .
\end{aligned}
$$

Таким образом, функции $W(t)$ и $R(t)$ удовлетворяют условиям леммы 1 с $M=0.04 \sqrt{k}$. Следовательно, на отрезке $T \leqslant t \leqslant T+H$ найдутся такие точки $t_{0}$ и $t_{1}$, что

$$
W\left(t_{0}\right)+R\left(t_{0}\right) \geqslant \frac{1}{8} M, \quad W\left(t_{1}\right)+R\left(t_{1}\right) \leqslant-\frac{1}{8} M .
$$

Пользуясь формулой леммы 2, запишем эти неравенства в виде

$$
\begin{aligned}
j_{0} & =\int_{-0.5 H \tau}^{0.5 H \tau}\left(\frac{\sin u}{u}\right)^{2} S\left(t_{0}+\frac{2 u}{\tau}\right) d u \\
& =W\left(t_{0}\right)+R\left(t_{0}\right)+O\left(\ln \tau+\frac{\ln t_{0}}{H \tau}\right) \geqslant \frac{1}{8} M+O(\ln \tau), \\
j_{1} & =\int_{-0.5 H \tau}^{0.5 H \tau}\left(\frac{\sin u}{u}\right)^{2} S\left(t_{1}+\frac{2 u}{\tau}\right) d u \\
& =W\left(t_{1}\right)+R\left(t_{1}\right)+O\left(\ln \tau+\frac{\ln t_{1}}{H \tau}\right) \leqslant-\frac{1}{8} M+O(\ln \tau) .
\end{aligned}
$$

Обозначим верхнюю и нижнюю грани функщии $S(t)$ на отрезке $T-H \leqslant t \leqslant T+2 H$ символами $M_{0}$ и $M_{1}$ соответственно. Тогда

$$
\begin{array}{ll}
j_{0} \leqslant M_{0} \int_{-0.5 H \tau}^{0.5 H \tau}\left(\frac{\sin u}{u}\right)^{2} d u<M_{0} \int_{-\infty}^{\infty}\left(\frac{\sin u}{u}\right)^{2} d u=\frac{\pi}{2} M_{0} \quad\left(M_{0}>0\right), \\
j_{1} \geqslant M_{1} \int_{-0.5 H \tau}^{0.5 H \tau}\left(\frac{\sin u}{u}\right)^{2} d u>M_{1} \int_{-\infty}^{\infty}\left(\frac{\sin u}{u}\right)^{2} d u=\frac{\pi}{2} M_{1} \quad\left(M_{1}<0\right) .
\end{array}
$$

Значит, при $r=0,1$

$$
(-1)^{r} M_{r}>\frac{2}{\pi}\left(\frac{1}{8} M+O(\ln \tau)\right)=\frac{1}{4 \pi} 0.04 \sqrt{k}+O(\ln \ln \ln T)>\frac{\varepsilon^{5 / 4}}{1000}\left(\frac{\ln T}{\ln \ln T}\right)^{1 / 3} .
$$

Теорема доказана. 
СлЕДСТВИЕ. При $t \rightarrow+\infty$

$$
S(t)=\Omega_{ \pm}\left(\sqrt[3]{\frac{\ln t}{\ln \ln t}}\right)
$$

Теорема 6. Пусть $T \geqslant T_{0}>0, \ln T<H \leqslant T$. Если гипотеза Римана верна, то справедливы неравенства:

$$
\sup _{T-H \leqslant t \leqslant T+2 H}( \pm S(t)) \geqslant 10^{-3} \sqrt{\frac{\ln T}{\ln \ln T}} .
$$

ДокАЗАТЕЛЬСТво. Положим

$$
k=\left[\frac{1}{7} \frac{\ln H}{\ln \ln H}\right], \quad \tau=2 \ln \ln H .
$$

Тогда вьполняются неравенства

$$
\begin{aligned}
& 2 k \ln k<2\left(\frac{1}{7} \frac{\ln H}{\ln \ln H}+1\right) \ln \ln H<(\ln H)^{4 / 3}=e^{2 \tau / 3} \\
& e^{3 k \tau}<\exp \left(6 \ln \ln H\left(\frac{1}{7} \frac{\ln H}{\ln \ln H}+1\right)\right)=H^{6 / 7}(\ln H)^{6}
\end{aligned}
$$

и, как следствие, оценки интегралов $I_{k}$ и $J_{k}$ леммы 3 :

$$
\begin{gathered}
I_{k}>(0.0025 k)^{k} H-H^{6 / 7}(\ln H)^{6}>(0.04 \sqrt{k})^{2 k} H, \\
\left|J_{k}\right|<e^{3 k \tau} e^{1.5 \tau}<H^{6 / 7}(\ln H)^{9}<0.5(0.04 \sqrt{k})^{2 k+1} H .
\end{gathered}
$$

По следствию из леммы 1 , на отрезке $T \leqslant t \leqslant T+H$ найдутся такие точки $t_{0}$ и $t_{1}$, что $W\left(t_{0}\right) \geqslant M / 8, W\left(t_{1}\right) \leqslant-M / 8$, где $M=0.04 \sqrt{k}$. Согласно лемме 2 , эти неравенства могут быть записаны в виде

$$
\begin{aligned}
& j_{0}=\int_{-0.5 H \tau}^{0.5 H \tau}\left(\frac{\sin u}{u}\right)^{2} S\left(t_{0}+\frac{2 u}{\tau}\right) d u \geqslant \frac{1}{8} M+O\left(\ln \tau+\frac{\ln t_{0}}{H \tau}\right)=\frac{1}{8} M+O(\ln \tau), \\
& j_{1}=\int_{-0.5 H \tau}^{0.5 H \tau}\left(\frac{\sin u}{u}\right)^{2} S\left(t_{1}+\frac{2 u}{\tau}\right) d u \leqslant \frac{1}{8} M+O\left(\ln \tau+\frac{\ln t_{1}}{H \tau}\right)=-\frac{1}{8} M+O(\ln \tau) .
\end{aligned}
$$

Обозначая верхнюю и нижнюю грани функции $S(t)$ на отрезке $T-H \leqslant t \leqslant T+2 H$ символами $M_{0}$ и $M_{1}$ и пользуясь тем, что $j_{0}<\pi M_{0} / 2, j_{1}>-\pi M_{1} / 2$ (см. доказательство теоремы 5), заключаем, что

$$
\begin{aligned}
(-1)^{r} M_{r} & >\frac{2}{\pi}\left(\frac{1}{8} M+O(\ln \tau)\right) \\
& =\frac{0.04}{4 \pi} \sqrt{\frac{\ln H}{7 \ln \ln H}}\left(1+O\left(\sqrt{\frac{\ln \ln H}{\ln H}} \ln \ln \ln H\right)\right)>10^{-3} \sqrt{\frac{\ln H}{\ln \ln H}} .
\end{aligned}
$$

Теорема доказана.

СлЕДСТВИЕ. Если гипотеза Римана верна, то при $t \rightarrow+\infty$

$$
S(t)=\Omega_{ \pm}\left(\sqrt{\frac{\ln t}{\ln \ln t}}\right) .
$$




\section{Замечания}

Различные способы доказательства неравенств теоремы 1 были предложены фон Мангольдтом в 1905 г. (см. [17]), Р. Бэклундом [18] в 1914 г. и Дж. Литтлвудом [29] в 1924 г. Теорема 2 также принадлежит Дж. Литтлвуду [29].

В настоящее время наилучшая оценка величины $|S(t)|$, при условии, что справедлива гипотеза Римана, принадлежит К. Рамачандре и А. Санкаранарайанану [32]:

$$
\pi|S(t)|<(3.51588780218300 \ldots+\varepsilon) \frac{\ln t}{\ln \ln t}, \quad t>t_{0}(\varepsilon)>0 .
$$

Теоремы 3 и 4 доказаны Г. Крамером [33] и Дж. Литтлвудом [29]. В основу изложения доказательств этих утверждений положены $\S \S 5$ и 6 гл. 5 книги Е. Титчмарша [20].

Первые омега-теоремы для функции $S(t)$ были получены в 1913 г. Г. Бором и Э. Ландау [17] и в 1925 г. Дж. Литтлвудом [34]. Используемые в главе III методы доказательства омега-теорем были разработаны А. Сельбергом [27] и уточнены К. Тсангом [30]. В частности, А. Сельбергом получены соотношения:

$$
S(t)=\Omega_{ \pm}\left((\ln t)^{1 / 3}(\ln \ln t)^{-7 / 3}\right) .
$$

Теоремы 5 и 6 принадлежат К. Тсангу [30] и Х. Монтгомери [35] соответственно. Большие значения $S(t)$ на коротком промежутке $(T, T+H], H>\ln T$, изучаются в [36].

Отметим, что в ходе больших вычислений, проведенных А. Одлыжко, не было обнаружено ни одного значения $t$, для которого $|S(t)|>3$ (см. [37; с. 27]).

Медленньй рост функций $\ln t, \ln \ln t$ в области, где в настоящее время могут быть вычислены значения $S(t)\left(|t| \leqslant 10^{23}\right)$, не дает возможности "угадать" правильньй порядок величин $\sup _{t \leqslant T}( \pm S(t))$. Согласно гипотезе $\mathrm{X}$. Монтгомери [35],

$$
S(t)=O\left(\sqrt{\frac{\ln t}{\ln \ln t}}\right) .
$$

В то же время А. Одлыжко в [37] высказал предположение, что

$$
S(t)=\Omega(\sqrt{\ln t}),
$$

но вместе с этим

$$
S(t)=O\left((\ln t)^{1 / 2+o(1)}\right) .
$$

В пользу этой гипотезы свидетельствует и условная теорема Я. Мозера [38], согласно которой на всяком интервале $(T, 2 T)$ существует не менее $c T \ln T$ промежутков $\left(\gamma, \gamma^{\prime}\right)$ таких, что

$$
|S(t)|<c_{1}(\ln T)^{1 / 2}(\ln \ln T)^{2 / 3}, \quad \gamma<t<\gamma^{\prime} .
$$

Для доказательства этого утверждения используется недоказанный в настоящее время факт: если $n(T)$ - число пар соседних ординат $\gamma, \gamma^{\prime}$ нулей дзета-функции, $T<$ $\gamma, \gamma^{\prime} \leqslant 2 T$, расстояние между которьми не превосходит $(\ln T)^{-2}$, то $n(T)=o(T \ln T)$.

Соотношение

$$
S_{1}(t)=\Omega_{ \pm}\left((\ln t)^{1 / 2-\varepsilon}\right)
$$


было доказано с использованием гипотезы Римана Дж. Литтлвудом [29] в 1924 г. Этот результат можно уточнить и заменить в приведенном соотношении множитель $(\ln t)^{-\varepsilon}$ на $(\ln \ln t)^{-3 / 2}$.

Первые безусловные омега-теоремы для функции $S_{1}(t)$ были получены А. Сельбергом $[27]$ :

$$
S_{1}(t)=\Omega_{-}\left((\ln t)^{1 / 3}(\ln \ln t)^{-10 / 3}\right), \quad S_{1}(t)=\Omega_{+}\left((\ln t)^{1 / 2}(\ln \ln t)^{-4}\right) .
$$

В настоящее время наиболее точными безусловными результатами являются теоремы Тсанга [25], [39]:

$$
S_{1}(t)=\Omega_{-}\left((\ln t)^{1 / 3}(\ln \ln t)^{-4 / 3}\right), \quad S_{1}(t)=\Omega_{+}\left((\ln t)^{1 / 2}(\ln \ln t)^{-3 / 2}\right) .
$$

Таким образом, отказ от использования гипотезы Римана не приводит к потери точности $\Omega_{+}$-теоремы для $S_{1}(t)$.

Омега-теоремы для разности $S(t+h)-S(t)$ рассмотрены в [30], [35].

\section{Приложение}

ЛЕмма 1 (функциональное уравнение $\zeta(s)$ ). Функиия $\xi(s)$, определяемая равенством

$$
\xi(s)=s(s-1) \pi^{-s / 2} \Gamma\left(\frac{s}{2}\right) \zeta(s)
$$

является целой и удовлетворяет соотношениям

$$
\xi(s)=1+s(s-1) \int_{1}^{+\infty}\left(x^{s / 2-1}+x^{-s / 2-1 / 2}\right) \omega(x) d x=\xi(1-s)=\bar{\xi}(1-\bar{s})
$$

әде

$$
\omega(x)=\sum_{n=1}^{+\infty} e^{-\pi x n^{2}} .
$$

Доказательство см. в [28; с. 17-20].

ЛЕмма 2 (симметрия нулей $\zeta(s))$. Функиия $\xi(s)$ представляется в виде бесконечного произведения

$$
\xi(s)=e^{B s} \prod_{\varrho}\left(1-\frac{s}{\varrho}\right) e^{s / \varrho}
$$

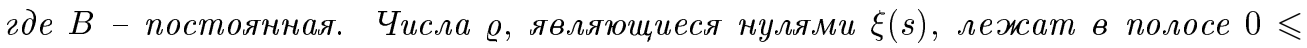
$\operatorname{Re} s \leqslant 1$ и расположсены симметрично относительно критической прямой $и$

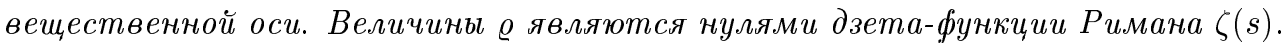

Доказательство см. в [28; с. 29-30]. 
ЛЕмма 3 (принцип аргумента). Пусть функиия $f(z)$ аналитична внутри области $D$ всюду, кроме конечного числа полюсов, непрерьвна на границе $C$ этой области и не обращается на $С$ в нуль. Пусть, кроме того, $f^{\prime}(z)$ непрерывна на $C$. Тогда разность между числом нулей и числом полюсов әтой функции внутри $D$ равна числу оборотов вектора $w$ при обходе кривой $\Gamma$, соответствующей $C$ при отображении $w=f(z)$, или, что то же самое, приращению аргумента $f(z)$ при изменении $z$ вдоль контура $C$, деленному на $2 \pi$.

Доказательство см. в [40; с. 87-88].

Лемма 4 (формула Стирлинга). При $0<\delta<\pi u|\arg s| \leqslant \pi-\delta$ имеет место формула:

$$
\ln \Gamma(s)=\left(s-\frac{1}{2}\right) \ln s-s+\ln \sqrt{2 \pi}+\int_{0}^{+\infty} \frac{\varrho(u) d u}{u+s}, \quad \text { дде } \quad \varrho(u)=1 / 2-\{u\} .
$$

Доказательство см. в [41; с. 54].

ЛЕмма 5 (теорема Литтлвуда). Пусть $C$ - контур прямоугольника, ограниченного прямиьми $x=x_{1}, x=x_{2}, y=y_{1}, y=y_{2}$, где $x_{1}<x_{2}, y_{1}<y_{2}$. Пусть $f(z)$ аналитична, не обращается в нуль на $С$ и мероморфна внутри С. Положим $F(z)=\ln f(z), z=x+i y$, определив логарифм следуюшим образом: начинаем с какой-либо непрерьвной ветви $\ln f(z)$ на прямой $x=x_{2}$ и получаем значение $\ln f(x+i y)$ из $\ln f\left(x_{2}+i y\right)$ путем непрерывного изменения вдоль прямой $y=\mathrm{const}$ до тех пор, пока не окажется $f(x+i y)=0$ или $f(x+i y)=\infty$. Таким образом, на конечном множестве отрезков, параллельных оси $x$, функция $\ln f(z)$ может быть не определена. Тогда имеет место соотношение:

$$
\begin{aligned}
2 \pi \int_{x_{1}}^{x_{2}} \nu(x) d x= & \int_{y_{1}}^{y_{2}}\left(\ln \left|f\left(x_{1}+i t\right)\right|-\ln \left|f\left(x_{2}+i t\right)\right|\right) d t \\
& +\int_{x_{1}}^{x_{2}}\left(\arg f\left(\sigma+i y_{2}\right)-\arg f\left(\sigma+i y_{1}\right)\right) d \sigma,
\end{aligned}
$$

где $\nu(x)$ - разность между числом нулей и числом полюсов $f(z)$ в области $x<\operatorname{Re} z \leqslant x_{2}, y_{1}<\operatorname{Im} z \leqslant y_{2}$.

Доказательство см. в [42; с. 456].

Лемма 6 (формула Иенсена). Пусть функиия $f(z)$ аналитична в круге $|z| \leqslant R$ и не обращается в нуль в центре и на границе этого круга. Тогда имеет место формула

$$
\int_{0}^{R} \frac{n(r)}{r} d r=\frac{1}{2 \pi} \int_{0}^{2 \pi} \ln \left|f\left(R e^{i \varphi}\right)\right| d \varphi-\ln |f(0)|,
$$

где $n(r)$ - число нулей $f(z)$ в круге $|z| \leqslant r$.

Доказательство см. в [43; с. 339]. 
Лемма 7 (теорема Адамара о трех кругах). Пусть $0<r_{1}<r_{2}$ u функиия $f(z)$ однозначна и аналитична в круге $|z| \leqslant r_{2}$. Если

$$
M(r)=\max _{|z|=r}|f(z)|,
$$

то при любом $r, r_{1} \leqslant r \leqslant r_{2}$, имеет место оченка

$$
M(r) \leqslant\left(M\left(r_{1}\right)\right)^{\frac{\ln \left(r_{2} / r\right)}{\ln \left(r_{2} / r_{1}\right)}}\left(M\left(r_{2}\right)\right)^{\frac{\ln \left(r / r_{1}\right)}{\ln \left(r_{2} / r_{1}\right)}} .
$$

Доказательство см. в [43; с. 379-380].

ЛЕмма 8 (неравенство Гёльдера). Пусть $u_{\nu}, v_{\nu} \geqslant 0$ для $\nu=1,2, \ldots, N, \alpha>0$, $\beta>0, \alpha+\beta=1$. Тогда

$$
\sum_{\nu=1}^{N} u_{\nu} v_{\nu} \leqslant\left(\sum_{\nu=1}^{N} u_{\nu}^{1 / \alpha}\right)^{\alpha}\left(\sum_{\nu=1}^{N} v_{\nu}^{1 / \beta}\right)^{\beta} .
$$

СлЕДСТВия.

1) $\left(\sum_{\nu=1}^{N} u_{\nu} v_{\nu}\right)^{2} \leqslant\left(\sum_{\nu=1}^{N} u_{\nu}^{2}\right)\left(\sum_{\nu=1}^{N} v_{\nu}^{2}\right) \quad$ (неравенство Коши);

2) $\left(\sum_{\nu=1}^{N} u_{\nu} v_{\nu}\right)^{k} \leqslant\left(\sum_{\nu=1}^{N} u_{\nu}\right)^{k-1}\left(\sum_{\nu=1}^{N} u_{\nu} v_{\nu}^{k}\right)$;

3) $\left(\sum_{\nu=1}^{N} u_{\nu}\right)^{k} \leqslant N^{k-1} \sum_{\nu=1}^{N} u_{\nu}^{k}$

Доказательство см. в [41; с. 85].

\section{СПИСОК ЛИТЕРАТУРЫ}

[1] А. А. Карацуба. Дзета-функция Римана и ее нули // УМН. 1985. Т. 40. № 5. С. 19-70.

[2] А. А. Карацуба. Распределение простых чисел // УМН. 1990. Т. 45. № 5. С. 81-140.

[3] A. Fujii. On the zeros of Dirichlet $L$-function. I // Trans. Amer. Math. Soc. 1974. V. 196. P. 225-235.

[4] A. Fujii. On the distribution of the zeros of the Riemann zeta function in short intervals // Bull. Amer. Math. Soc. 1975. V. 81. P. 139-142.

[5] A. Fujii. Gram's law for the zeta zeros and the eigenvalues of Gaussian unitary ensembles // Proc. Japan Acad. Ser. A Math. Sci. 1987. V. 63. № 10. P. 392-395.

[6] A. Fujii. On the distribution of the zeros of the Riemann zeta function in short intervals // Proc. Japan Acad. Ser. A Math. Sci. 1990. V. 66. № 3. P. 75-79.

[7] A. Fujii. On the gaps between the consecutive zeros of the Riemann zeta function // Proc. Japan Acad. Ser. A Math. Sci. 1990. V. 66. № 4. P. 97-100.

[8] A. Ghosh. On Riemann's zeta-function - sign changes of $S(T) / /$ Recent Progress in Analytic Number Theory. V. 1. New York: Academic Press, 1981. P. 25-46.

[9] A. Ghosh. On the Riemann zeta function - mean value theorems and the disribution of $|S(T)| / /$ J. Number Theory. 1983. V. 17. № 1. P. 93-102. 
[10] Y. Mozer. The Riemann hypothesis and asymmetry in the behavoir of positive and negative values of the function $S_{1}(t) / /$ Acta Math. Univ. Comenian. 1986. V. 48/49. P. 63-80.

[11] J. H. Mueller. On the Riemann zeta function $\zeta(s)$ - gaps between sign changes of $S(t) / /$ Mathematika. 1982. V. 29. № 2. P. 264-269.

[12] A. Selberg. On the remainder in the formula for $N(T)$, the number of zeros of $\zeta(s)$ in the strip $0<t<T / /$ Avh. Norske Vid. Akad. Oslo. I. 1944. №1. P. 1-27.

[13] A. Selberg. Old and new conjectures and results about a class of Dirichlet series // Proceedings of the Amalfi Conference on Analytic Number Theory (Maiori, 1989). Salerno: Univ. di Salerno, 1992. P. 365-387.

[14] E. C. Titchmarsh. On the remainder in the formula for $N(T)$, the number of zeros of $\zeta(s)$ in the strip $0<t<T / /$ Proc. London Math. Soc. (2). 1928. Part 6. V. 27. P. 449-458.

[15] K.-M. Tsang. An improved $\Omega_{+}$theorem for $S_{1}(t) / /$ Quart. J. Math. Oxford. Ser. (2). 1991. V. 42. № 168. P. 501-513.

[16] Б. Риман. О числе простых чисел, не превышающих данной величины // Б. Риман. Сочинения. М.: ОГИЗ, 1948. С. 216-224.

[17] H. Bohr, E. Landau. Beiträge zur Theorie der Riemannschen Zetafunktion // Math. Ann. 1913. V. 74. № 1. P. 3-30.

[18] R.-J. Backlund. Sur les zéros de la fonction $\zeta(s)$ de Riemann // Comptes rendus. Acad. Sci Paris. 1914. V. 158. P. 1979-1981.

[19] E. C. Titchmarsh. The zeros of the Riemann zeta-function // Proc. Roy. Soc. London (A). 1935. V. 151. P. 234-255.

[20] Е. К. Титчмарш. Теория дзета-функции Римана. М.: ИЛ, 1953.

[21] А. А. Карацуба. Плотностная теорема и поведение аргумента дзета-функции Римана // Матем. заметки. 1996. Т. 60. № 3. С. 448-449.

[22] А. А. Карацуба. О функции $S(t) / /$ Изв. РАН. Сер. матем. 1996. Т. 60. № 5. С. 27-56.

[23] М. А. Королёв. О числе перемен знака функции $S(t)$ на коротком промежутке // Докл. PAH. 2002. Т. 382. № 4. C. 446-447.

[24] М. А. Королёв. Об аргументе дзета-функции Римана на критической прямой // Труды МИАН. 2002. Т. 239. С. 215-238.

[25] М. А. Королёв. О поведении функции $S(t)$ на коротких промежутках // Докл. РАН. 2003. № 5. C. 588-589.

[26] М. А. Королёв. Об аргументе дзета-функции Римана на критической прямой // Изв. РАН. Сер. матем. 2003. Т. 67. № 2. С. 21-60.

[27] A. Selberg. Contributions to the theory of the Riemann zeta-function // Arch. Math. Naturvid. 1946. V. 48. № 5. P. 89-155.

[28] С. М. Воронин, А. А. Карацуба. Дзета-функция Римана. М.: Физматлит, 1994.

[29] J. E. Littlewood. On the zeros of the Riemann zeta-function // Proc. Cambridge Philos. Soc. 1924. V. 22. P. 295-318.

[30] K.-M. Tsang. Some $\Omega$-theorems for the Riemann zeta-function // Acta Arith. 1986. V. 46. № 4. P. 369-395.

[31] D. A. Goldston. On the function $S(T)$ in the theory of the Riemann zeta-function // J. Number Theory. 1987. V. 27. № 2. P. 149-177.

[32] K. Ramachandra, A. Sankaranarayanan. On some theorems of Littlewood and Selberg, I // J. Number Theory. 1993. V. 44. № 3. P. 281-291.

[33] H. Cramér. Über die Nullstellen der Zetafunktion // Math. Z. 1918. V. 2. P. 237-241.

[34] J. E. Littlewood. On the Riemann zeta-function // Proc. London Math. Soc. 1925. V. 24. № 2. P. $175-201$.

[35] H. L. Montgomery. Extreme values of the Riemann zeta function // Comment. Math. Helv. 1977. V. 52. № 4. P. 511-518.

[36] М. А. Королёв. О болшших значениях функции $S(t)$ на коротких промежутках // Изв. PAH. 2005. T. 69. № 1. C. 115-124.

[37] A. M. Odlyzko. The $10^{20}$-th zero of the Riemann zeta function and 70 million of its neighbors // Preprint. Murray Hill, NJ: AT\&T Bell Laboratories, 1987. 
[38] Я. Мозер. О функции $S(t)$ в теории дзета-функции Римана // Acta Arith. 1976. V. 30. № 2. P. $145-158$.

[39] K.-M. Tsang. The large values of the Riemann zeta-function // Mathematika. 1993. V. 40. № 2. P. 203-214.

[40] М. А. Лаврентьев, Б. В. Шабат. Методы теории функций комплексного переменного. M.: Наука, 1965.

[41] А. А. Карацуба. Основы аналитической теории чисел. М.: Наука, 1983.

[42] К. Прахар. Распределение простых чисел. М.: Мир, 1967.

[43] Г. Полиа, Г. Сегё. Задачи и теоремы из анализа. Ч. 1. М.: Наука, 1978.

Математический институт им. В. А. Стеклова РАН

Поступила в редакцию 